\title{
A Staphylococcus aureus lipoteichoic acid (LTA) derived structural variant with two diacylglycerol residues
}

\author{
A. Stadelmaier, ${ }^{a}$ I. Figueroa-Perez, ${ }^{\text {a }}$ S. Deininger, ${ }^{b}$ S. von Aulock, ${ }^{b}$ \\ T. Hartung ${ }^{\mathrm{b}}$ and R. R. Schmidt $\mathrm{a}^{\mathrm{a}, *}$

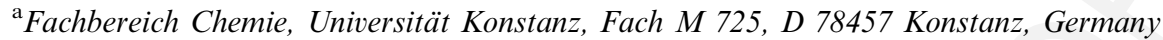 \\ ${ }^{\mathrm{b}}$ Fachbereich Biologie, Universität Konstanz, Fach M 668, D 78457 Konstanz, Germany
}

\begin{abstract}
Based on 1,2 $O$ isopropylidene $s n$ glycerol five chiral building blocks containing differently modified glycerol residues were required for the synthesis of the target molecule 2. One of these building blocks is diacylglyceryl $\beta$ gentiobioside carrying a phosphite residue at $6 \mathrm{~b} O$ position. Ligation of these five building blocks led to the desired glycerol phosphate backbone to which D alanyl residues were attached, thus generating after $O$ deprotection the target molecule $\mathbf{2}$, a bisamphiphilic structural variant of Staphylococcus aureus LTA. This compound displayed higher potency in terms of cytokine release by human blood leukocytes than the monoamphiphilic variant LTA.
\end{abstract}

\section{1. Introduction}

For Gram-negative bacteria, LPS is well established as the crucial stimulus of the innate immune system, as injection of LPS into mice causes all known symptoms of sepsis. ${ }^{1}$ There is now good evidence that lipoteichoic acid (LTA) from the cytoplasmic membrane of Grampositive bacteria is an immunostimulatory Gram-positive counterpart to LPS. ${ }^{2-6}$ The most frequently isolated Gram-positive pathogen that causes infections is Staphylococcus aureus, ${ }^{7}$ and the development of antibiotic resistance in this species is a big problem. ${ }^{8,9}$ Therefore, alternatives and adjuvants to antibiotics are required. To identify these, it is important to understand the pathophysiology of this bacterial infection.

The structure of the $S$. aureus LTA is shown in Scheme $1 .^{3,10,11}$ For unequivocal bioactivity assignment, besides an improved isolation procedure, ${ }^{11}$ the chemical synthesis of the structurally closely related compound 1a (Scheme 1) was decisive. ${ }^{3-5,12}$ This compound contains the hydrolytically labile D-alanine residues in the required ratio with other substituents at a hexameric glycerophosphate backbone. Compound 1a exhibited

$\overline{\text { Keywords: }}$ Bacteria; Gram positive; Lipoteichoic acid; Bisamphiphilic; Synthesis; Cytokine release.

* Corresponding author. Tel.: +49 753188 2538; fax: +49 753188 3135; e mail: Richard.Schmidt@uni konstanz.de essentially the same biological activity in terms of initiation of cytokine release by human blood leukocytes as found for the natural product. Whereas the replacement of the D-alanine residues by L-alanine residues as in $\mathbf{1 b}$ led to almost complete loss of biological activity. ${ }^{4}$ Further structural modifications of LTA 1a, as for instance deletion of the gentiobiose moiety or replacement of the hydrolytically labile ester bond to the D-alanyl residue by a stable amide bond, led only to minor decrease of induction of cytokine release by human blood leukocytes. $^{5,13}$ Thus, the importance of the D-alanyl residues was displayed. However, none of the investigated structural modifications so far led to an increased induction of cytokine release. For this, optimal presentation of the hydrophilic part of LTA to the receptor should be of utmost importance; therefore, bisamphiphilic compound 2 (Scheme 2) having two diacylglycerol gentiobioside residues at each end of the glycerolphosphate backbone was designed. It was hypothesized that with two lipid anchors, possibly within the same membrane, the epitope presentation should be supported due to sterically improved accessibility to the D-alanyl and the $\alpha$ O-linked $N$-acetylglucosamine residues. ${ }^{14}$

\section{Results and discussion}

The retrosynthesis of compound $\mathbf{2}$ is shown in Scheme 2. Disintegrations lead to building blocks 3 to 8 which 


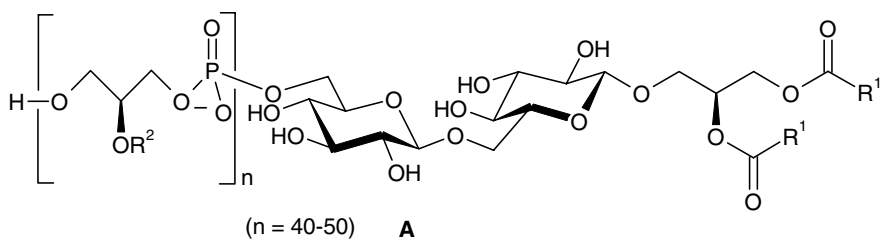<smiles>[R]=C[C@H]([NH3+])[C@@H](C)C(C)=O</smiles>

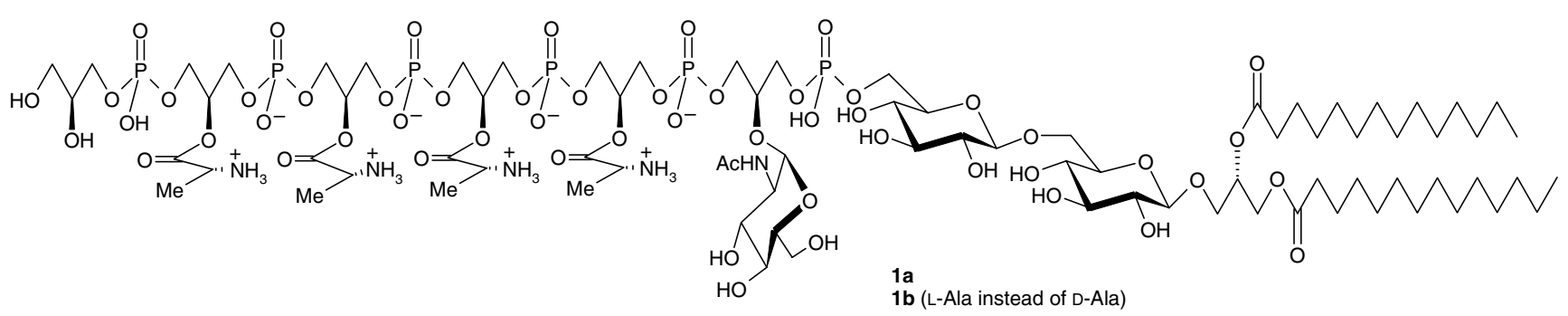

Scheme 1. General structure A of lipoteichoic acid (LTA) from Staphylococcus aureus (A) and structure of closely related compound 1a and its diastereoisomer $\mathbf{1 b}$.

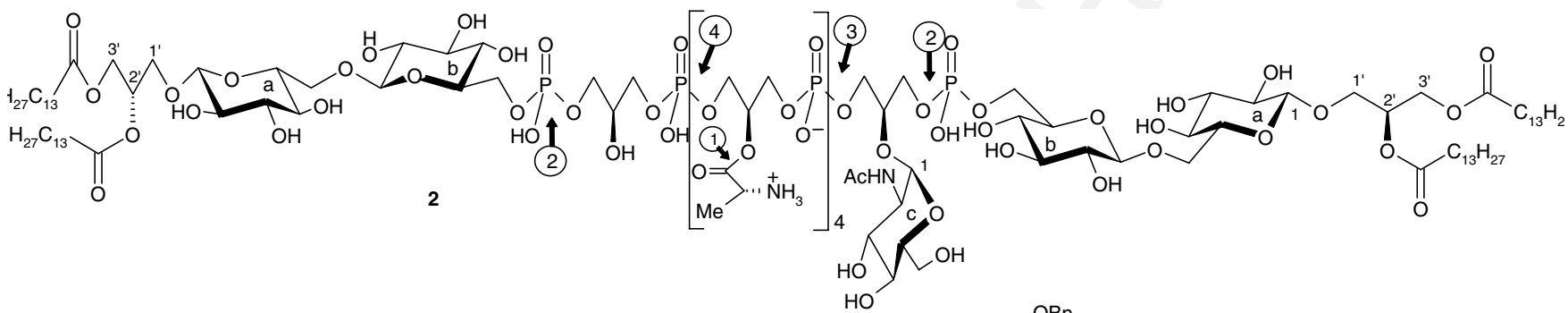

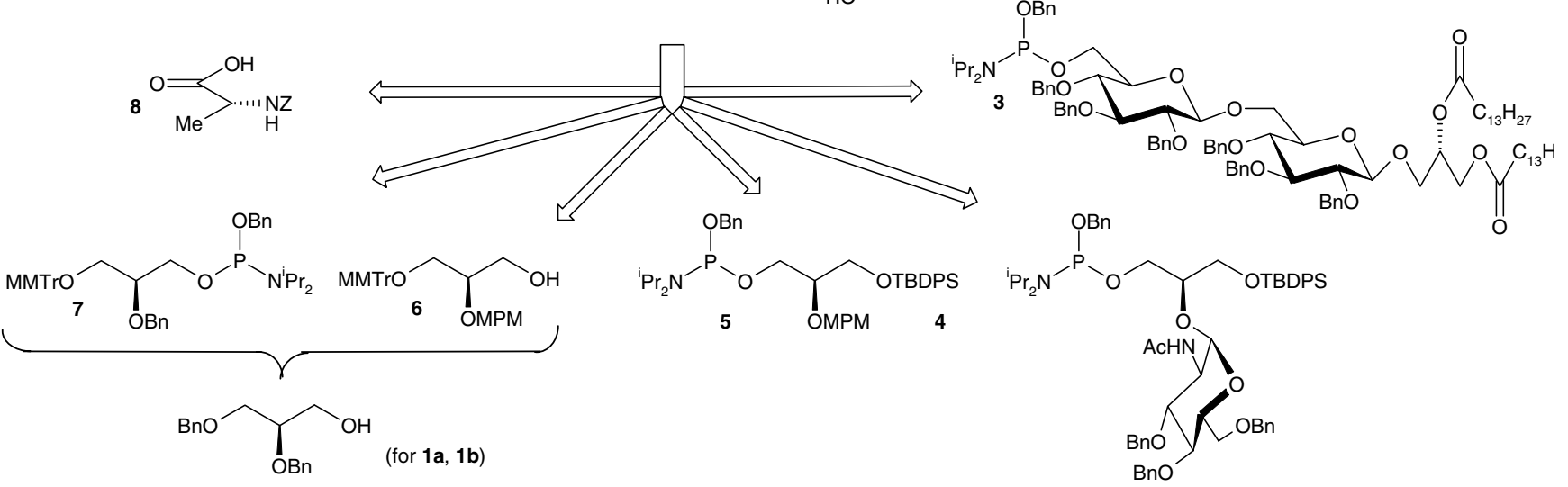

Scheme 2. Structure of target molecule 2 and required building blocks $3 \mathbf{8}$ for the synthesis

consider the presence of diacylglyceryl gentiobioside, glyceryl 2- $N$-acetylamino-2-deoxy- $\alpha$-D-glucopyranoside, D-alanylated glycerol and 2-O-unsubstituted glycerol residues, respectively, and their sequence specific linkage via phosphorus diester bonds. Also the most important aspect, the lability of the D-alanyl residues, which are readily cleaved at $\mathrm{pH} 8.5$, is taken into account: as temporary protecting groups in building blocks 5 and 6 4methoxyphenylmethyl (MPM) groups are chosen; they can be selectively cleaved by oxidation after completion of the backbone synthesis. Ensuing attachment of D-alanyl residues with Z-protected alanine 8 and then complete O-debenzylation will provide the target molecule 2. Building blocks 35 and, as substitute for 6 and 7, 2,3- di- $O$-benzyl-sn-glycerol, ${ }^{4}$ were successfully employed in the synthesis of LTAs 1a, b. Because this work has not been reported in detail, ${ }^{15}$ the syntheses of compounds 1a, b will also be described.

\subsection{Synthesis of building block 3}

Benzoylation of gentiobiose with benzoyl chloride in pyridine gave an $\alpha$-, $\beta$-mixture of per-O-benzoylated compound 9 (Scheme 3). Treatment of 9 with hydrazinium acetate in DMF permitted chemoselective removal of the anomeric $O$-benzoyl group furnishing 1-O-unprotected compound 10. Reaction of $\mathbf{1 0}$ with trichloroacetonitrile in the presence of 1,8-diazabicyclo[5.4.0]undec-7-ene 


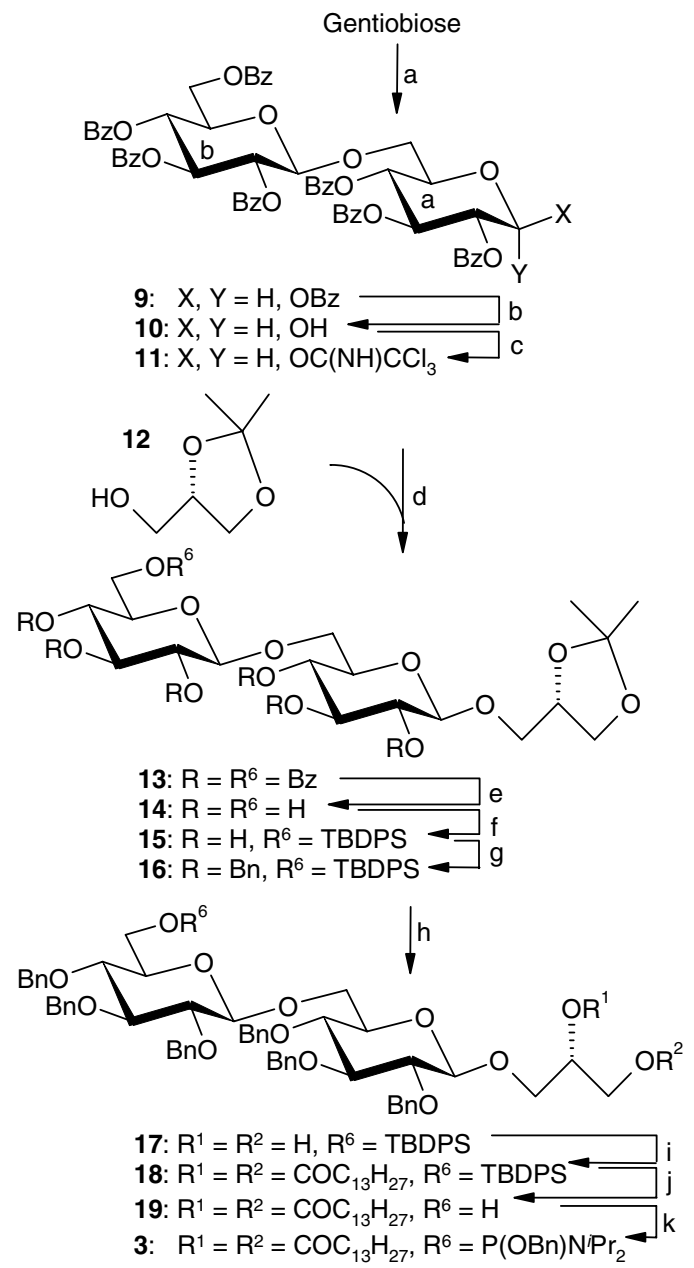

Scheme 3. Synthesis of building block 3. Reagents and conditions: (a) $\mathrm{Bz} \mathrm{Cl}$, pyr, $40{ }^{\circ} \mathrm{C}$ (98\%); (b) $\mathrm{N}_{2} \mathrm{H}_{4} \cdot \mathrm{HOAc}, 50{ }^{\circ} \mathrm{C}$, DMF (78\%); (c) $\mathrm{CCl}_{3} \mathrm{CN}$, DBU, $\mathrm{CH}_{2} \mathrm{Cl}_{2}(97 \%)$; (d) $\mathrm{BF}_{3} \cdot \mathrm{OEt}_{2}, \mathrm{CH}_{2} \mathrm{Cl}_{2}, \quad 30^{\circ} \mathrm{C}(80 \%)$; (e) $\mathrm{NaOMe}, \mathrm{MeOH}$ (qu); (f) TBDPS Cl, pyr, $15^{\circ} \mathrm{C}(91 \%$ ); (g) $\mathrm{Bn} \mathrm{Br}$, $\mathrm{NaH}, \mathrm{DMF}(66 \%)$; (h) $\mathrm{HOAc}, \mathrm{THF} / \mathrm{H}_{2} \mathrm{O}, 80^{\circ} \mathrm{C}(78 \%)$; (i) myristoyl chloride, $\mathrm{NEt}_{3}$, THF, $50{ }^{\circ} \mathrm{C}(81 \%)$; (j) TBAF, HOAc, THF, $40{ }^{\circ} \mathrm{C}$ (76\%); (k) BnOP $\left(\mathrm{N}^{i} \mathrm{Pr}_{2}\right)_{2}$, tetrazole, $\mathrm{THF} / \mathrm{CH}_{2} \mathrm{Cl}_{2}(79 \%)$.

(DBU) as base afforded trichloroacetimidate $\mathbf{1 1}$ as a 1:1 anomeric mixture. Glycosylation of 1,2-O-isopropylidene-sn-glycerol $(\mathbf{1 2})^{16}$ with $\mathbf{1 1}$ in the presence of $\mathrm{BF}_{3} \cdot \mathrm{OEt}_{2}$ as activator gave exclusively $\beta$-glycoside $\mathbf{1 3}$ in $80 \%$ yield. Complete O-debenzoylation with sodium methoxide in methanol afforded compound $\mathbf{1 4}$ which was regioselectively silylated at the primary hydroxy group with tert-butyldiphenylsilyl (TBDPS) chloride in pyridine to give $6 \mathrm{~b}-\mathrm{O}$-protected compound $\mathbf{1 5}$. Following O-benzylation with benzyl bromide and sodium hydride as base in DMF as solvent afforded fully O-protected gentiobioside 16. Acid-catalyzed O-deisopropylidenation $(\rightarrow$ 17) and then treatment with myristoyl chloride in the presence of triethylamine as base led to introduction of two myristoyl residues furnishing compound 18. 6b-O-Desilylation with tetrabutylammonium fluoride (TBAF) in the presence of acetic acid in THF as solvent gave 6b-O-unprotected compound 19 which on reaction with benzyloxy-bis(diisopropylamino)-phosphane ${ }^{17}$ in the presence of tetrazole as catalyst furnished building block $\mathbf{3}$.

\subsection{Synthesis of LTA $1 \mathrm{a}$ and its diastereomer $1 \mathrm{~b}$}

With building block 3 and the previously synthesized glycerophosphate oligomer $\mathbf{2 0},{ }^{13}$ possessing the required protecting group array for regioselective chain extension and for following D-alanyl residue attachment, the synthesis of $\mathbf{1 a}, \mathbf{b}$ could be readily completed (Scheme 4). Ligation of $\mathbf{3}$ and $\mathbf{2 0}$ in the presence of tetrazole and then oxidation with tert-butylhydroperoxide gave phosphate linked intermediate $\mathbf{2 1}$, which contains the backbone of the target molecule. Treatment of 21 with ceric(IV) ammonium nitrate (CAN) liberated four of the glycerol hydroxy groups affording compound 22 . Attachment of the Z-protected D-alanyl residues was performed with excess 8 in the presence of (benzotriazol-1-yloxy)tripyrrolidinophosphonium hexafluorophosphate $^{18} \quad$ (PyBOP)/N-methyl-imidazole as condensing agent to give the fully protected target molecule 23a. Hydrogenolysis with Pearlman's catalyst ${ }^{19}$ in a mixture of $\mathrm{CH}_{2} \mathrm{Cl}_{2} / \mathrm{MeOH} / \mathrm{H}_{2} \mathrm{O}$ (5:5:1) furnished the desired final product 1a after hydrophobic interaction (HI) chromatography on actylsepharose in $47 \%$ yield. The structural assignment was based on MS and NMR data and comparison with naturally occurring material. ${ }^{3}$ Compound $\mathbf{2 2}$ was similarly transformed with Z-protected L-alanine via $\mathbf{2 3 b}$ into $\mathbf{1 b}$.

\subsection{Synthesis of building blocks 6 and 7}

Previously described 1-O-(tert-butyldiphenylsilyl)-2-O(4-methoxybenzyl)-sn-glycerol (24), ${ }^{13}$ obtained from 1,2-O-isopropylidene-sn-glycerol, was treated with monomethoxytrityl (MMTf) chloride in $\mathrm{CH}_{2} \mathrm{Cl}_{2}$ /pyridine to afford fully O-protected glycerol derivative $\mathbf{2 5}$ (Scheme 5). Regioselective $O$-desilylation with TBAF in THF afforded the 1-O-unprotected building block 6 .

Treatment of previously described 3-O-allyl-1-O-(tertbutyldiphenylsilyl)-sn-glycerol (26) ${ }^{13}$ with benzyl bromide in DMF afforded per-O-protected glycerol 27. ODeallylation with Wilkinson's catalyst ${ }^{20}$ in the presence of DBU as base in ethanol furnished the 3-O-propenyl derivative which was cleaved with $1 \mathrm{M} \mathrm{HCl}$ in acetone to afford 3-O-unprotected 28. Reaction with MMTr-Cl in $\mathrm{CH}_{2} \mathrm{Cl}_{2}$ /pyridine furnished fully protected glycerol 29 which on treatment with TBAF in THF led to 1-Ounprotected glycerol 30. Phosphitylation with benzyloxy-bis(diisopropylamino)phosphane ${ }^{17}$ led to the desired phosphite derivative 7 .

\subsection{Synthesis of target molecule 2}

The assembly of target molecule 2 from building blocks 38 was performed stepwise in solution starting with building block 6 (Scheme 6). Phosphitylation of $\mathbf{6}$ with 5 in the presence of tetrazole and then oxidation with tert-butylhydroperoxide gave phosphate intermediate 31 which was O-desilylated with TBAF in THF as solvent to furnish $\mathbf{3 2}$ with a free hydroxy group as acceptor for the next reaction cycles for chain extension. This way, with 5 intermediates 3335 were obtained. Following cleavage of the MMTr group with camphersulfonic acid (CSA) in methanol afforded $\mathbf{3 6}$ which on phosphitylation 

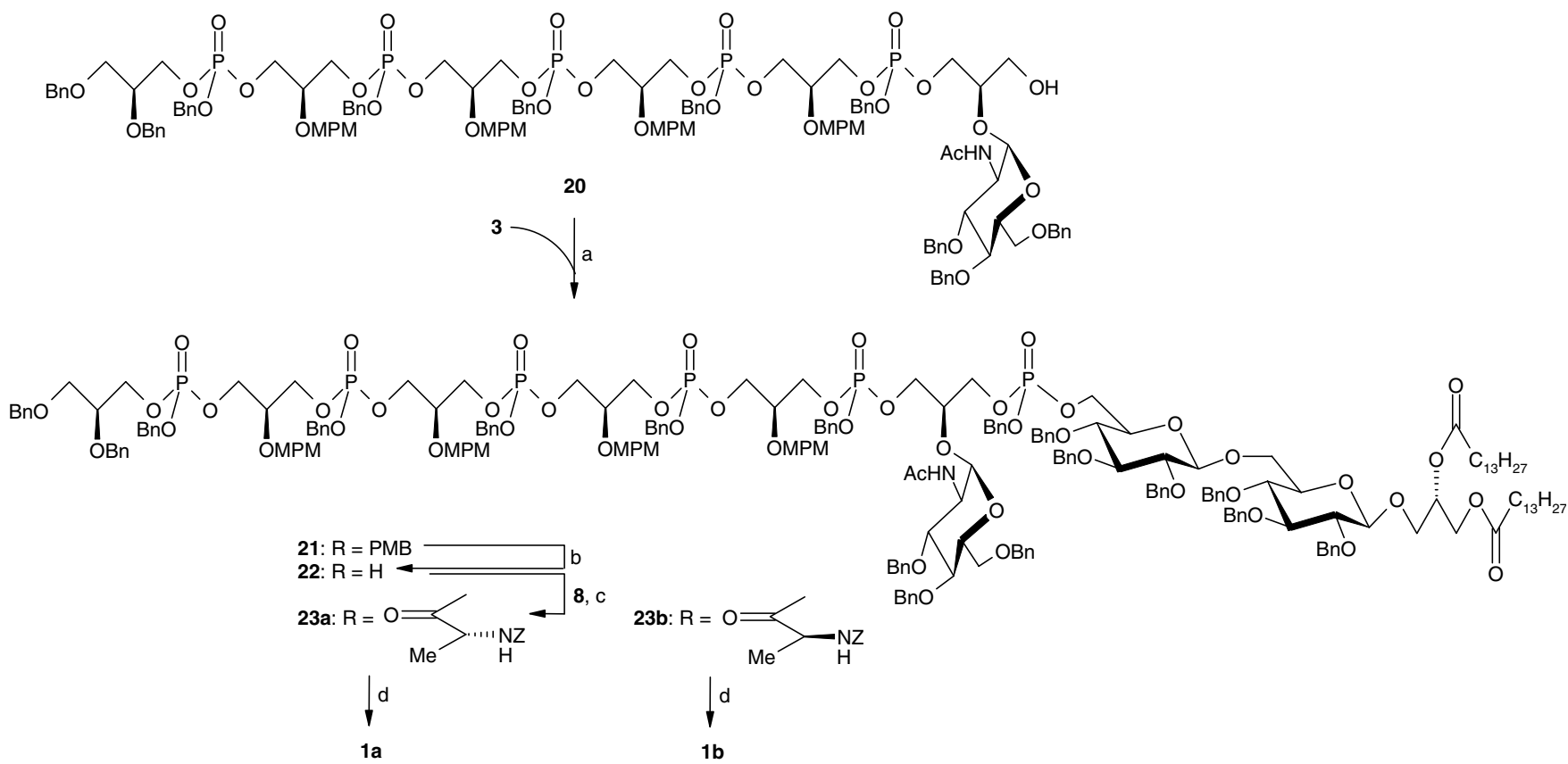

Scheme 4. Synthesis of compounds $\mathbf{1 a}$ and $\mathbf{1 b}$. Reagents and conditions: (a) tetrazole, $\mathrm{CH}_{2} \mathrm{Cl}_{2} ; t \mathrm{BuO}_{2} \mathrm{H}(75 \%) ;\left(\right.$ b) $\mathrm{CAN}, \mathrm{MeCN} / \mathrm{Tol} / \mathrm{H}_{2} \mathrm{O}, \quad 10{ }^{\circ} \mathrm{C}$ $\rightarrow$ rt (67\%); (c) PyBOP, Me Im, $\mathrm{CH}_{2} \mathrm{Cl}_{2}\left(23 \mathrm{a}, 70 \%\right.$; 23b, 62\%); (d) $\mathrm{Pd}(\mathrm{OH})_{2} / \mathrm{C}, \mathrm{H}_{2}, \mathrm{CH}_{2} \mathrm{Cl}_{2} / \mathrm{MeOH} / \mathrm{H}_{2} \mathrm{O}(\mathbf{1 a}, 47 \% ; \mathbf{1 b}, 40 \%)$.<smiles>[R9]CC(CO[R6]Sc1ccccc1)O[M]</smiles>

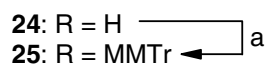<smiles>C1CCCCC1</smiles>

6<smiles></smiles>

26: $R^{1}=$ All, $R^{2}=H$ 27: $R^{1}=$ All, $R^{2}=B n$ 28: $R^{1}=H, R^{2}=B n$ 29: $\mathrm{R}^{1}=\mathrm{MMTr}, \mathrm{R}^{2}=\mathrm{Bn} \longrightarrow \mathrm{e}$<smiles>C=ICC(CO)OCc1ccccc1</smiles>

30: $\mathrm{R}=\mathrm{H}$ 7: $\mathrm{R}=\mathrm{P}(\mathrm{OBn}) \mathrm{N} P r_{2} \sim \mathrm{g}$

Scheme 5. Synthesis of building blocks 6 and 7. Reagents and conditions: (a) MMTr Cl, $\mathrm{CH}_{2} \mathrm{Cl}_{2} / \mathrm{pyr}$ (98\%); (b) TBAF, THF (96\%); (c) Bn Br, NaH, DMF (72\%); (d) $\left(\mathrm{Ph}_{3} \mathrm{P}\right)_{3} \mathrm{RhCl}, \mathrm{DBU}$, EtOH; $\mathrm{HCl}, \mathrm{Me}_{2} \mathrm{CO}, 70{ }^{\circ} \mathrm{C}(76 \%)$; (e) $\mathrm{MMTr} \mathrm{Cl}, \mathrm{CH}_{2} \mathrm{Cl}_{2} / \mathrm{pyr}(98 \%)$; (f) TBAF, THF (98\%); (g) $\mathrm{BnO}\left({ }^{i} \mathrm{Pr}_{2} \mathrm{~N}\right)_{2} \mathrm{P}$, tetrazole, THF/ $\mathrm{CH}_{2} \mathrm{Cl}_{2}$ (qu).

with building block 7, oxidation and then TBDPS group cleavage led to compounds 37 and 38. Then phosphitylation with building block 4 and oxidation gave pentaphosphate intermediate 39. Selective deprotection of the primary hydroxy groups first by treatment with CSA in methanol $(\rightarrow \mathbf{4 0})$ and then with TBAF in THF afforded compound 41 which was available to attachment of building block 3 at each terminus. To this end, $\mathbf{4 1}$ was treated with tetrazole under standard conditions to afford compound $\mathbf{4 2}$ in good yield (Scheme 7), which contains the desired glycerophosphate backbone of target molecule 2. For the attachment of the D-alanyl residues, the four MPM groups were selectively removed by treatment with CAN in an acetonitrile/toluene/water mixture as solvent affording compound 43 in $76 \%$ yield. D-Alanylation was performed with triethylammonium salt of $\mathbf{8}$ in the presence of $N$-methyl-imidazole and PyBOP as condensing agent furnishing fully protected target molecule 44 in $49 \%$ yield. Hydrogenolytic O-debenzylation (i.e. cleavage of $27 O$-benzyl groups) was performed with Pearlman's catalyst ${ }^{19}$ in a dichloromethane/methanol/ water mixture as solvent. The crude product was purified by $\mathrm{HI}$ chromatography to afford target molecule $\mathbf{2}$ in $32 \%$ yield. Compound $\mathbf{2}$ was structurally ascertained as all intermediates by NMR and MS data and most intermediates also by elemental analyses.

\section{Biological activity}

The evaluation of the biological activity of compound 2 was performed in comparison with compound 1a (Fig. 1), which induces a similar pattern of cytokine release as natural $S$. aureus LTA. ${ }^{3,4}$ Measurement of initiation of cytokine release (A, TNF $\alpha$; B, IL-8) by human blood leukocytes displayed that bisamphiphilic $\mathbf{2}$ is more potent than monoamphiphilic LTA 1a by a factor of about 10. Hence, the hypothesis that bisamphiphilic LTA analogues should exhibit stronger biological activity than monohomophilic LTA 1a was proven to be correct. 

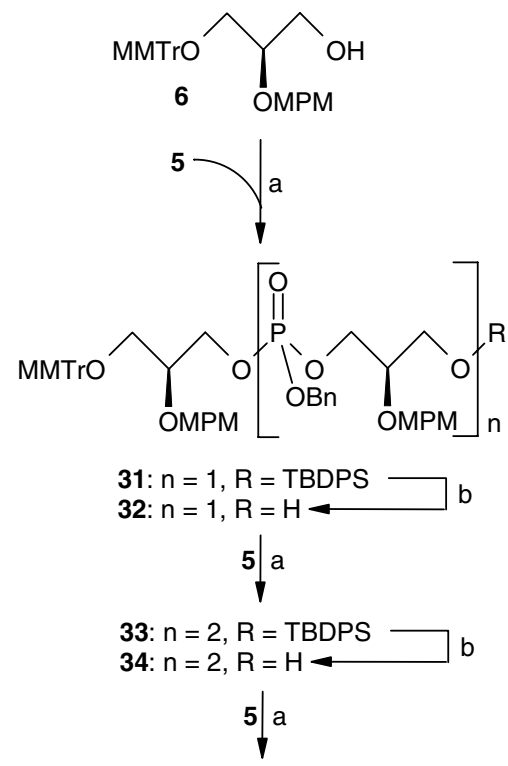

Scheme 6. Synthesis of glycerolphosphate oligomer 41. Reagents and conditions: (a) tetrazole, $\mathrm{CH}_{2} \mathrm{Cl}_{2} ; t$ BuO $\mathrm{Bu}_{2}(\mathbf{3 1}, 98 \% ; \mathbf{3 3}, 96 \% ; \mathbf{3 5}, 94 \% ; \mathbf{3 7}$, $96 \%$; 39, 96\%); (b) TBAF, THF (32, 82\%; 34, 91\%; 36, 92\%; 38, 95\%; 41, 85\%); (c) CSA, MeOH (92\%).

\section{Conclusion}

In conclusion, retrosynthesis of bisamphiphilic structural variant 2 of $S$. aureus LTA led to six building blocks out of which five required differently modified chiral glycerol residues. They readily permitted the assembly of compound 2 . The backbone construction was based on the ligation of different glycerol phosphites possessing, similar to nucleotide synthesis, temporary O-protecting groups permitting sequence specific chain extension. Hence, based on this synthesis design ready access not only to monoamphiphilic $S$. aureus LTA 1a, which is also described in detail, but also to a multitude of structural variants of LTA is available. As anticipated, bisamphiphilic compound $\mathbf{2}$ is more potent in terms of induction of cytokine release in human leukocytes than natural LTA and its shortened version 1a.

\section{Experimental}

\subsection{General remarks}

Solvents were dried according to standard procedures. NMR spectroscopic measurements were performed at $22{ }^{\circ} \mathrm{C}$ with Bruker DRX600 and Bruker AC250 instruments. TMS or the resonances of the deuterated solvents were used as internal standard. $\mathrm{CDCl}_{3}(\delta=7.24 \mathrm{ppm})$ was used as external standard; $85 \%$ of phosphoric acid was used as external standard for ${ }^{31} \mathrm{P}$ spectra. MALDI mass spectra were recorded with a Kratos Kompact Maldi II spectrometer; 2,5-dihydroxybenzoic acid (DHB) or $p$-nitroaniline and $\mathrm{NaI}$ were used as matrices for positive measurements, and trihydroxyacetophenone (THAP) was used as matrix for negative mode measurements. Optical rotations were measured with a Perkin Elmer polarimeter $241 / \mathrm{MS}$ in a $1-\mathrm{dm}$ cell at $22^{\circ} \mathrm{C}$. 


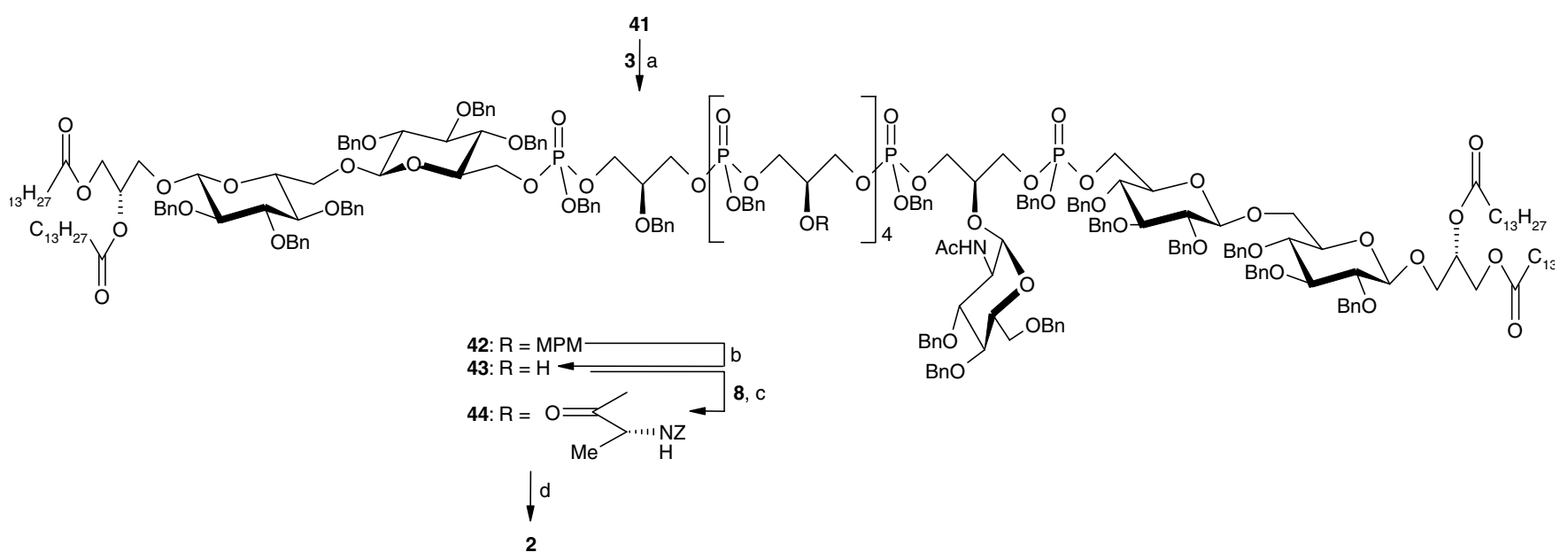

Scheme 7. Synthesis of target molecule 2. Reagents and conditions: (a) tetrazole, $\mathrm{CH}_{2} \mathrm{Cl}_{2} ; t \mathrm{BuO}_{2} \mathrm{H}(71 \%) ;\left(\right.$ b) $\mathrm{CAN}, \mathrm{MeCN} / \mathrm{Tol} / \mathrm{H}_{2} \mathrm{O}, \quad 10{ }^{\circ} \mathrm{C} \rightarrow \mathrm{rt}$ (76\%); (c) PyBOP, Me Im, $\mathrm{CH}_{2} \mathrm{Cl}_{2}(49 \%)$; (d) $\mathrm{Pd}(\mathrm{OH})_{2} / \mathrm{C}, \mathrm{H}_{2}, \mathrm{CH}_{2} \mathrm{Cl}_{2} / \mathrm{MeOH} / \mathrm{H}_{2} \mathrm{O}(16 \%)$.
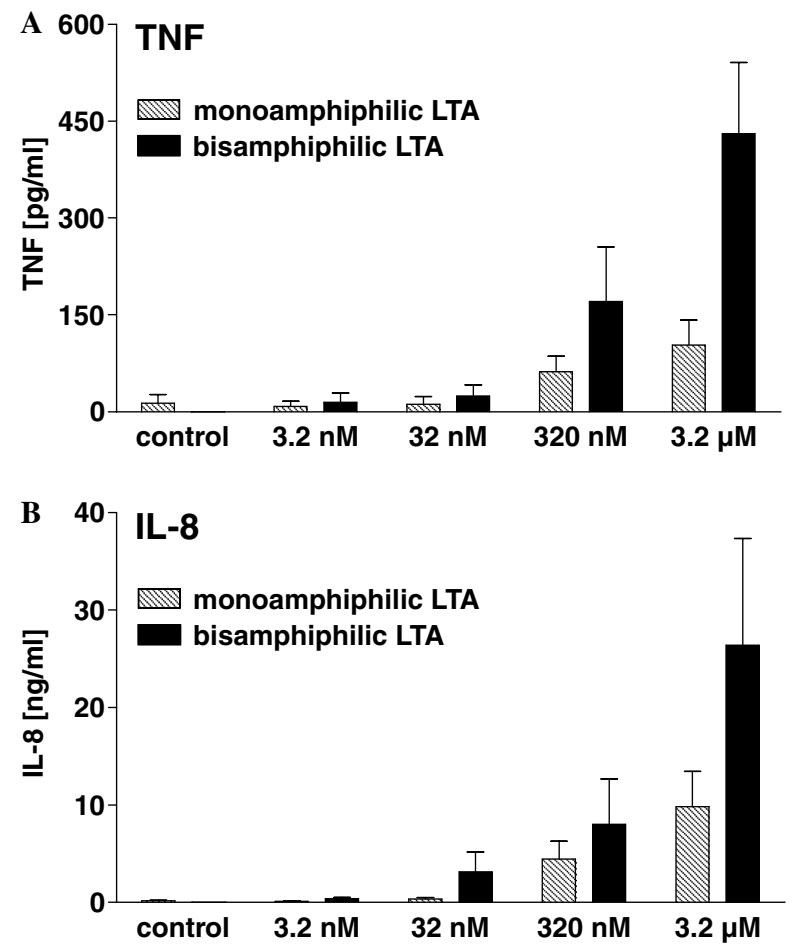

Figure 1. Concentration dependence of TNF $\alpha$ (A) and IL 8 (B) release in human whole blood in response to 1a and $\mathbf{2}$ as measured by ELISA. Data are means $( \pm$ SEM) of blood from four donors. SEM, standard error of the mean.

Thin-layer chromatography (TLC) was performed on Merck silica gel $60 \mathrm{~F}_{254}$ plastic plates. Compounds were visualized by treatment with a solution of $\left(\mathrm{NH}_{4}\right)_{6} \mathrm{Mo}_{7} \mathrm{O}_{24} \cdot 4 \mathrm{H}_{2} \mathrm{O}(20 \mathrm{~g})$ and $\mathrm{Ce}\left(\mathrm{SO}_{4}\right)_{2}(0.4 \mathrm{~g})$ in $10 \%$ sulfuric acid $(400 \mathrm{~mL})$. Flash chromatography was performed on J. T. Baker silica gel $60(0.0400 .063 \mathrm{~mm})$ at a pressure of 0.3 bar. Target molecules were purified by Hydrophobic Interaction Chromatography on octylsepharose as stationary phase and as elution phase was used as a gradient of propanol (15 60\%) in $0.1 \mathrm{M}$ ammonium acetate buffer ( $\mathrm{pH} 4.8)$.

\subsection{General procedure for phosphate formation}

The alcohol and the phosphane (1.2 equiv) were coevaporated with dry $\mathrm{CH}_{2} \mathrm{Cl}_{2}$ and dried in high vacuum for $1 \mathrm{~h}$. The mixture was dissolved in dry $\mathrm{CH}_{2} \mathrm{Cl}_{2}$ and tetrazole ( 2.5 equiv, previously dried for $1 \mathrm{~h}$ in high vacuum) was added. The reaction mixture was stirred for $1.5 \mathrm{~h}$ at room temp (TLC control), and after this time $t-\mathrm{BuO}_{2} \mathrm{H}$ (1.3 equiv of $5.5 \mathrm{M}$ solution in decane) was added. The reaction mixture was stirred for another $3045 \mathrm{~min}$ and diluted with EtOAc, washed with saturated $\mathrm{NaHCO}_{3}$ solution. The organic phase was dried over $\mathrm{MgSO}_{4}$ and evaporated in vacuo. Purification by flash chromatography on silica gel gave the desired product.

\subsection{General procedure for the removal of tert-butyldi- phenylsilyl protecting group}

The silylated compound was dissolved in THF (p.a. quality) and treated with TBAF (1.2 equiv of $1 \mathrm{M}$ solution in THF). The reaction mixture was stirred for 30 $45 \mathrm{~min}$ at room temp (monitoring by TLC). After this time, the reaction mixture was diluted with EtOAc and washed with saturated $\mathrm{NH}_{4} \mathrm{Cl}$ solution and water. The organic phase was dried over $\mathrm{MgSO}_{4}$ and the solvent evaporated in vacuo. Flash chromatography on silica gel gave the desired compound.

\subsection{6-O-(2,3,4,6-Tetra- $O$-benzoyl- $\beta$-D-glucopyranosyl)- 1,2,3,4-tetra- $O$-benzoyl- $\alpha / \beta$-D-glucopyranose (9)}

Gentiobiose (14.13 g, $41.28 \mathrm{mmol})$ was dissolved in pyridine $(375 \mathrm{~mL})$, benzoyl chloride was added $(50 \mathrm{~mL}$, $0.48 \mathrm{~mol}$ ) and the reaction mixture was stirred at $40{ }^{\circ} \mathrm{C}$ overnight. The solvent was removed and the crude material purified by flash chromatography (petroleum ether/ EtOAc, 2:1) to give 9 in 98\% yield (48 g) as a white solid. TLC (petroleum ether/EtOAc 3:2): $R_{\mathrm{f}}=0.45 ; \alpha: \beta \approx 1: 1$. $[\alpha]_{\mathrm{D}}+15.8\left(c 1, \mathrm{CHCl}_{3}\right) .{ }^{1} \mathrm{H}$ NMR $\left(250 \mathrm{MHz}, \mathrm{CDCl}_{3}\right):$ $\delta=3.404 .17,4.184 .30,4.354 .65(\mathrm{~m}, 6 \mathrm{H}, 6 \mathrm{a}, 6 \mathrm{~b}, 5 \mathrm{a}$, $5 \mathrm{~b}-\mathrm{H}), 5.05\left(\mathrm{~d}, 0.5 \mathrm{H}, J_{1,2}=7.8 \mathrm{~Hz}, 1 \mathrm{~b}-\mathrm{H}\right), 5.405 .78$, $5.886 .01(\mathrm{~m}, 6 \mathrm{H}, 2 \mathrm{a}, 2 \mathrm{~b}, 3 \mathrm{a}, 3 \mathrm{~b}, 4 \mathrm{a}, 4 \mathrm{~b}-\mathrm{H}), 6.15$ (d, 
$\left.0.5 \mathrm{H}, J_{1,2}=8.2 \mathrm{~Hz}, 1 \mathrm{a}\right), 7.207 .65,7.768 .15(\mathrm{~m}, 35 \mathrm{H}$, $\mathrm{Ph}$ ). MALDI-MS (positive Mode, Matrix DHB, dioxane): $[\mathrm{M}+\mathrm{Na}]^{+}, \quad m / z=1198.2$; found: $m / z=1198.6$, $[\mathrm{M}+\mathrm{K}]^{+}, m / z=1214.3$; exp.: $m / z=1215.7 . \mathrm{C}_{68} \mathrm{H}_{54} \mathrm{O}_{19}$ (1175.2): Calcd: C, 69.50; H, 4.63. Found: C, 69.15; H, 4.74 .

\subsection{6-O-(2,3,4,6-Tetra- $O$-benzoyl- $\beta$-D-glucopyranosyl)- 2,3,4-tri- $O$-benzoyl- $\alpha / \beta$-D-glucopyranose (10)}

A solution of $9(48 \mathrm{~g}, 40.85 \mathrm{mmol})$ in dry DMF $(425 \mathrm{~mL})$ was heated to $50{ }^{\circ} \mathrm{C}$ and hydrazinium acetate was added $(5.4 \mathrm{~g})$. After $3.5 \mathrm{~h}$, the solution was diluted with $\mathrm{CH}_{2} \mathrm{Cl}_{2}$ and washed with water. The organic phase was dried over $\mathrm{MgSO}_{4}$ and the solvent evaporated. The crude material was purified by flash chromatography (toluene/EtOAc 7:1) to give $\mathbf{1 0}$ in $78 \%$ of yield $(34.12 \mathrm{~g})$ as a colourless solid. TLC (toluene/EtOAc 6:1): $R_{\mathrm{f}}=0.30 .[\alpha]_{\mathrm{D}}+30.6\left(c 0.34, \mathrm{CHCl}_{3}\right) .{ }^{1} \mathrm{H} \mathrm{NMR}$ $\left(250 \mathrm{MHz}, \mathrm{CDCl}_{3}\right): \delta=3.14$ (br s, $\left.1 \mathrm{H}, \mathrm{OH}\right), 3.62$ $4.24,4.384 .59,4.634 .82(\mathrm{~m}, 6 \mathrm{H}, 6 \mathrm{a}, 6 \mathrm{~b}, 5 \mathrm{a}, 5 \mathrm{~b}-\mathrm{H})$, $4.98\left(\mathrm{~d}, 1 \mathrm{H}, J_{1,2}=7.6 \mathrm{~Hz}, 1 \mathrm{~b}-\mathrm{H}\right), 5.096 .22(\mathrm{~m}, 7 \mathrm{H}$, 1a, 2a, 2b, 3a, 3b, 4a, 4b-H), 7.20 7.65, 7.74 8.17 (m, $30 \mathrm{H}, \mathrm{Ph}) . \mathrm{C}_{61} \mathrm{H}_{50} \mathrm{O}_{18} 0.5 \mathrm{H}_{2} \mathrm{O}$ (1080.1): Calcd: $\mathrm{C}$, 67.84;, H, 4.76. Found: C, 67.80; H, 4.75.

\section{6. $O$-[6- $O$-(2,3,4,6-tetra- $O$-benzoyl- $\beta$-D-glucopyrano- syl)-2,3,4-tri- $O$-benzoyl- $\alpha / \beta$-D-glucopyranosyl] trichloro- acetimidate (11)}

Compound $10(32.11 \mathrm{~g}, 29.98 \mathrm{mmol})$ was dissolved in dry $\mathrm{CH}_{2} \mathrm{Cl}_{2}(450 \mathrm{~mL})$, and $\mathrm{Cl}_{3} \mathrm{CCN}(30.5 \mathrm{~mL}, 0.3 \mathrm{~mol})$ was added; subsequently DBU was added $(0.45 \mathrm{~mL})$. After $2 \mathrm{~h}$, the solvent was removed in vacuo and the rest was purified by flash chromatography (petroleum ether/ EtOAc $2: 1,+1 \% \mathrm{Et}_{3} \mathrm{~N}$ ) to give $\mathbf{1 1}$ in $97 \%$ of yield $(35.35 \mathrm{~g})$ as a slightly yellow foam. TLC (petroleum ether/EtOAc 2:1): $R_{\mathrm{f}}=0.33$. $[\alpha]_{\mathrm{D}}=+26\left(c=1, \mathrm{CHCl}_{3}\right)$. ${ }^{1} \mathrm{H}$ NMR $\left(250 \mathrm{MHz}, \mathrm{CDCl}_{3}\right): \delta=3.404 .17,4.184 .30$, $4.354 .65(\mathrm{~m}, 6 \mathrm{H}, 6 \mathrm{a}, 6 \mathrm{~b}, 5 \mathrm{a}, 5 \mathrm{~b}-\mathrm{H}), 5.05(\mathrm{~d}, 0.5 \mathrm{H}$, $\left.J_{1,2}=7.8 \mathrm{~Hz}, 1 \mathrm{~b}-\mathrm{H}\right), 5.405 .78,5.886 .01(\mathrm{~m}, 6 \mathrm{H}, 2 \mathrm{a}$, $2 \mathrm{~b}, 3 \mathrm{a}, 3 \mathrm{~b}, 4 \mathrm{a}, 4 \mathrm{~b}-\mathrm{H}), 6.15\left(\mathrm{~d}, 0.5 \mathrm{H}, J_{1,2}=8.2 \mathrm{~Hz}, 1 \mathrm{a}\right)$, $7.207 .65,7.768 .15$ (m, $35 \mathrm{H}, \mathrm{Ph}) . \mathrm{C}_{63} \mathrm{H}_{50} \mathrm{O}_{18} \mathrm{NCl}_{3} 0.5$ $\mathrm{H}_{2} \mathrm{O}$ (1224.5): Calcd: C. 61.80; $\mathrm{Hm} 4.201 ; \mathrm{N}: 1.14$; found: $\mathrm{C}: 61.68, \mathrm{H}: 4.17, \mathrm{~N}: 0.97$.

\subsection{1,2-O-Isopropylidene-3- $O$-[6- $O-(2,3,4,6$-tetra- $O$-ben- zoyl- $\beta$-D-glucopyranosyl)-2, 3, 4-tri- $O$-benzoyl- $\beta$-D-gluco- pyranosyl]-sn-glycerol (13)}

To a solution of $\mathbf{1 2}^{16}$ ( $4.2 \mathrm{~mL}, 1.2$ equiv) and donor $\mathbf{1 1}$ $(35 \mathrm{~g}, 28.8 \mathrm{mmol})$ in dry $\mathrm{CH}_{2} \mathrm{Cl}_{2}(450 \mathrm{~mL})$ molecular sieves $(3 \AA)$ were added and the reaction mixture was stirred for $15 \mathrm{~min}$ at $\mathrm{rt}$ under argon atmosphere. Subsequently the reaction mixture was cooled to $-30^{\circ} \mathrm{C}$ and $\mathrm{BF}_{3} \mathrm{Et}_{2} \mathrm{O}$ solution $(0.2$ equiv, $0.75 \mathrm{~mL}$ ) was added. After $20 \mathrm{~min}$, the reaction mixture was neutralized with $\mathrm{NEt}_{3}$, evaporated to half volume, rediluted with toluene, and the solvent was removed in vacuo. The crude material was purified by flash chromatography (petroleum ether/EtOAc 2:1, $+1 \% \mathrm{NEt}_{3}$ ) to give $\mathbf{1 3}$ in $80 \%$ of yield $(27.3 \mathrm{~g} ; \quad 23.04 \mathrm{mmol}) . \quad$ TLC (toluene/EtOAc 6:1): $R_{\mathrm{f}}=0.3 .[\alpha]_{\mathrm{D}}+3.6\left(c 1, \mathrm{CHCl}_{3}\right) .{ }^{1} \mathrm{H} \mathrm{NMR}(600 \mathrm{MHz}$,
$\left.\mathrm{CDCl}_{3}\right): \delta=1.22,1.26\left(2 \mathrm{~s}, 6 \mathrm{H}, \mathrm{C}\left(\mathrm{CH}_{3}\right)_{2}\right), 3.42(\mathrm{dd}, 1$ $\left.\mathrm{H}, J_{\text {gem }}=10.6 \mathrm{~Hz}, J_{\text {vic }}=5.6 \mathrm{~Hz}, 1^{\prime}-\mathrm{H}\right), 3.62(\mathrm{dd}, 1 \mathrm{H}$, $\left.J_{\text {gem }}=8.0 \mathrm{~Hz}, \quad J_{\text {vic }}=6.7 \mathrm{~Hz}, \quad 3^{\prime}-\mathrm{H}\right), 3.69(\mathrm{dd}, 1 \mathrm{H}$, $\left.J_{\text {gem }}=10.6 \mathrm{~Hz}, \quad J_{\text {vic }}=4.0 \mathrm{~Hz}, 1^{\prime}-\mathrm{H}\right), 3.81(\mathrm{dd}, 1 \mathrm{H}$, $\left.J_{\text {gem }}=8.1 \mathrm{~Hz}, \quad J_{\text {vic }}=6.5 \mathrm{~Hz}, 3^{\prime}-\mathrm{H}\right), 3.85(\mathrm{dd}, 1 \mathrm{H}$, $\left.J_{\text {gem }}=11.4 \mathrm{~Hz}, J_{\text {vic }}=7.6 \mathrm{~Hz}, 6 \mathrm{a}-\mathrm{H}\right), 3.964 .03(\mathrm{~m}, 1$ $\mathrm{H}, 5 \mathrm{a}-\mathrm{H}), 4.05\left(\mathrm{dd}, 1 \mathrm{H}, J_{\text {gem }}=11.4 \mathrm{~Hz}, J_{\text {vic }}=1.5 \mathrm{~Hz}\right.$, 6a-H), $4.094 .16(\mathrm{~m}, 1 \mathrm{H}, 5 \mathrm{~b}-\mathrm{H}), 4.42(\mathrm{dd}, 1 \mathrm{H}$, $\left.J_{\text {gem }}=12.1 \mathrm{~Hz}, J_{\text {vic }}=5.1 \mathrm{~Hz}, 6 \mathrm{~b}-\mathrm{H}\right), 4.59(\mathrm{dd}, 1 \mathrm{H}$, $\left.J_{\text {gem }}=12.1 \mathrm{~Hz}, \quad J_{\text {vic }}=2.9 \mathrm{~Hz}, 6 \mathrm{~b}-\mathrm{H}\right), 4.71(\mathrm{~d}, 1 \mathrm{H}$, $\left.J_{1,2}=7.9 \mathrm{~Hz}, 1 \mathrm{a}-\mathrm{H}\right), 4.97\left(\mathrm{~d}, 1 \mathrm{H}, J_{1,2}=7.8 \mathrm{~Hz}, 1 \mathrm{~b}-\mathrm{H}\right)$, $5.31\left(\mathrm{dd}, 1 \mathrm{H}, J_{4,5}=J_{4,3}=9.7 \mathrm{~Hz}, 4 \mathrm{a}-\mathrm{H}\right), 5.37(\mathrm{dd}, 1$ $\left.\mathrm{H}, J_{2,1}=8.1 \mathrm{~Hz}, J_{2,3}=9.6 \mathrm{~Hz}, 2 \mathrm{a}-\mathrm{H}\right), 5.50(\mathrm{dd}, 1 \mathrm{H}$, $\left.J_{2,1}=8.0 \mathrm{~Hz}, \quad J_{2,3}=9.6 \mathrm{~Hz}, 2 \mathrm{~b}-\mathrm{H}\right), 5.62(\mathrm{dd}, 1 \mathrm{H}$, $\left.J_{4,5}=J_{4,3}=9.7 \mathrm{~Hz}, \quad 4 \mathrm{~b}-\mathrm{H}\right), \quad 5.78 \quad(\mathrm{dd}, \quad 1 \quad \mathrm{H}$, $\left.J_{3,4}=J_{3,2}=9.6 \mathrm{~Hz}, \quad 3 \mathrm{a}-\mathrm{H}\right), \quad 5.89 \quad(\mathrm{dd}, \quad 1 \quad \mathrm{H}$, $\left.J_{3,4}=J_{3,2}=9.6 \mathrm{~Hz}, 3 \mathrm{~b}-\mathrm{H}\right), 7.177 .58,7.718 .05(\mathrm{~m}, 35$ $\mathrm{H}, \mathrm{Ph}) .{ }^{13} \mathrm{C}$ NMR $\left(150.9 \mathrm{MHz}, \mathrm{CDCl}_{3}\right): \delta=25.38$, $26.41\left(2 \mathrm{C}, \mathrm{C}\left(\mathrm{CH}_{3}\right)_{2}\right), 62.94$ (1 C, C-6b), 66.07 (1 C, C-3'), 68.51 (1 C, C-6a), 68.83 (1 C, C-1'), 69.51 (1 C, C-4b), 69.83 (1 C, C-4a), 71.67 (1 C, C-2a), 71.86 (1 C, C-2b), 72.28 (1 C, C-5b), 72.78 (2 C, C-3a, C-3b), 73.88 (1 C, C-5a), 74.09 (1 C, C-2'), 100.93 (1 C, C1a), 101.27 (1 C, C-1b), 128.24133 .47 (42 C, Ph), 165.04166 .06 (7 C, CO-Ph). MALDI-MS (positive Mode, Matrix DHB, THF): $[\mathrm{M}+\mathrm{Na}]^{+}, \mathrm{m} / z=1208.2$; found: $m / z=1208.9,[\mathrm{M}+\mathrm{K}]^{+}, \mathrm{m} / z=1224.3$; found: $\mathrm{m} /$ $z=1225.3 . \mathrm{C}_{67} \mathrm{H}_{60} \mathrm{O}_{20}$ (1185.2): Calcd: C, 67.90; $\mathrm{H}$, 5.10. Found: C, 67.69; H, 5.01.

\subsection{1,2-O-Isopropylidene-3- $O$-[6- $O$-(- $\beta$-D-glucopyrano-

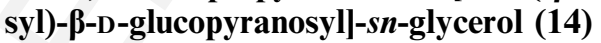

To a solution of $13(19.14 \mathrm{~g}, 16.15 \mathrm{mmol})$ in dry $\mathrm{MeOH}$ $(300 \mathrm{~mL})$ and dry $\mathrm{CH}_{2} \mathrm{Cl}_{2}(20 \mathrm{~mL})$ was added $\mathrm{NaH}$ $(0.81 \mathrm{~g}, 33.75 \mathrm{mmol})$ and stirred at rt. After $12 \mathrm{~h}$, the reaction mixture was neutralized with amberlite IR-120 $\mathrm{H}^{+}$, filtered and evaporated in vacuo with a little amount of $\mathrm{Et}_{3} \mathrm{~N}$. The solid was dissolved in a mixture of dioxane/ water and lyophilized, $\mathbf{1 4}$ was obtained in quantitative yield $(7.37 \mathrm{~g})$ as a yellow solid. TLC $($ EtOAc/ $\mathrm{MeOH}$ 5:2): $R_{\mathrm{f}}=0.28 .[\alpha]_{\mathrm{D}}-19.1 \quad\left(c=1, \mathrm{CHCl}_{3}\right) .{ }^{1} \mathrm{H} \mathrm{NMR}$ (600 MHz, $\left.\mathrm{CD}_{3} \mathrm{OD}\right): \delta=1.31,1.38\left(2 \mathrm{~s}, 6 \mathrm{H}, \mathrm{C}\left(\mathrm{CH}_{3}\right)_{2}\right)$, $3.143 .23(\mathrm{~m}, 2 \mathrm{H}, 2 \mathrm{a}, 2 \mathrm{~b}-\mathrm{H}), 3.26(\mathrm{~m}, 1 \mathrm{H}, 5 \mathrm{~b}-\mathrm{H}), 3.33$ (m, $2 \mathrm{H}, 3 \mathrm{a}, 3 \mathrm{~b}-\mathrm{H}), 3.423 .48(\mathrm{~m}, 1 \mathrm{H}, 5 \mathrm{a}-\mathrm{H}), 3.63$ (dd, $\left.1 \mathrm{H}, J_{1^{\prime}, 2^{\prime}}=6.0 \mathrm{~Hz}, J_{\mathrm{gem}}=10.5 \mathrm{~Hz}, 1^{\prime}-\mathrm{H}\right), 3.65(\mathrm{dd}, 1$ $\left.\mathrm{H}, J_{\text {gem }}=11.8 \mathrm{~Hz}, J_{\text {vic }}=5.2 \mathrm{~Hz}, 6 \mathrm{~b}-\mathrm{H}\right), 3.76(\mathrm{dd}, 1 \mathrm{H}$, $\left.J_{\text {gem }}=11.6 \mathrm{~Hz}, J_{\text {vic }}=5.9 \mathrm{~Hz}, 6 \mathrm{a}-\mathrm{H}\right), 3.81(\mathrm{dd}, 1 \mathrm{H}$, $\left.J_{\text {gem }}=8.4 \mathrm{~Hz}, J_{\text {vic }}=6.1 \mathrm{~Hz}, 3^{\prime}-\mathrm{H}\right), 3.86(\mathrm{dd}, 1 \mathrm{H}$, $\left.J_{\text {gem }}=11.3 \mathrm{~Hz}, \quad J_{\text {vic }}=1.6 \mathrm{~Hz}, 6 \mathrm{~b}-\mathrm{H}\right), 3.89(\mathrm{dd}, 1 \mathrm{H}$, $\left.J_{\text {gem }}=10.5 \mathrm{~Hz}, J_{\text {vic }}=5.5 \mathrm{~Hz}, 1^{\prime}-\mathrm{H}\right), 4.07(\mathrm{dd}, 1 \mathrm{H}$, $\left.J_{\mathrm{gem}}=8.3 \mathrm{~Hz}, \quad J_{\text {vic }}=6.5 \mathrm{~Hz}, 3^{\prime}-\mathrm{H}\right), 4.14(\mathrm{dd}, 1 \mathrm{H}$, $\left.J_{\text {gem }}=11.5 \mathrm{~Hz}, J_{\text {vic }}=1.7 \mathrm{~Hz}, 6 \mathrm{a}-\mathrm{H}\right), 4.29(\mathrm{~d}, 1 \mathrm{H}$, $\left.J_{1,2}=7.8 \mathrm{~Hz}, 1 \mathrm{a}-\mathrm{H}\right), 4.31\left(\mathrm{~m}, 1 \mathrm{H}, 2^{\prime}-\mathrm{H}\right), 4.36(\mathrm{~d}, 1 \mathrm{H}$, $\left.J_{1,2}=7.8 \mathrm{~Hz}, 1 \mathrm{~b}-\mathrm{H}\right) .{ }^{13} \mathrm{C} \mathrm{NMR}\left(150.9 \mathrm{MHz}, \mathrm{CD}_{3} \mathrm{OD}\right)$ : $\delta=25.62\left(1 \mathrm{C}, \mathrm{C}\left(\mathrm{CH}_{3}\right)_{2}\right), 27.09\left(1 \mathrm{C}, \mathrm{C}\left(\mathrm{CH}_{3}\right)_{2}\right), 62.75$ (1 C, C-6b), 67.75 (1 C, C-3'), 69.94 (1 C, C-6a), 71.4571.59 (3 C, C-1', C-4a/b, C-5b), 74.98, 75.10 (2 C, C2a, C-2b), 75.86 (1 C, C-2'), 77.06 (1 C, C-5a), 77.85, 78.02 (3 C, C-4a/b, C-3a, C-3b), 104.68 (2 C, C-1a, C$1 \mathrm{~b}), 104.92\left(1 \mathrm{C}, \mathrm{C}\left(\mathrm{CH}_{3}\right)_{2}\right)$. MALDI-MS (positive Mode, Matrix DHB, dioxane): $[\mathrm{M}+\mathrm{Na}]^{+}, \mathrm{m} / z=479.4$; found: $m / z=479.0,[\mathrm{M}+\mathrm{K}]^{+}, m / z=495.5$. Found: $m / z=494.8$. 
5.9. 1,2-O-Isopropylidene-3-O-[6-O-(6-O-tert-butyldiphe-

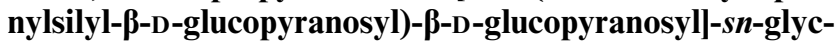
erol (15)

A solution of $14(7.35 \mathrm{~g}, 16.1 \mathrm{mmol})$ in pyridine $(150 \mathrm{~mL})$ was cooled to $-15^{\circ} \mathrm{C}$ and $5 \mathrm{~mL}$ TBDPS-Cl (1.2 equiv, $9.2 \mathrm{mmol}$ ) was added dropwise. The reaction mixture was stirred for $72 \mathrm{~h}$, quenched with $\mathrm{MeOH}$, evaporated in vacuo and coevaporated with toluene. After flash chromatography (EtOAc/MeOH 4:1), product 15 (10.2 g, 91 $\%)$ was obtained. TLC (EtOAc/MeOH 3:2): $R_{\mathrm{f}}=0.60$. $[\alpha]_{\mathrm{D}}-25.5\left(c 1, \mathrm{CHCl}_{3}\right) .{ }^{1} \mathrm{H}$ NMR $\left(250 \mathrm{MHz}, \mathrm{CD}_{3} \mathrm{OD}\right)$ : $\delta=1.02(\mathrm{~s}, 9 \mathrm{H}, t-\mathrm{Bu}), 1.31,1.39\left(2 \mathrm{~s}, 6 \mathrm{H}, \mathrm{C}\left(\mathrm{CH}_{3}\right)_{2}\right)$, $3.184 .42(\mathrm{~m}, 19 \mathrm{H}), 7.3$ 7.46, $7.637 .80(\mathrm{~m}, 10 \mathrm{H}, \mathrm{Ph})$. MALDI-MS (positive Mode, Matrix DHB, dioxane): $[\mathrm{M}+\mathrm{Na}]^{+}, m / z=717.85$; found: $m / z=717.1,[\mathrm{M}+\mathrm{K}]^{+}$, $\mathrm{m} / \mathrm{z}=733.95$; found: $\mathrm{m} / \mathrm{z}=733.1$.

5.10. 1,2- $O$-Isopropylidene-3- $O$-[6- $O$ - $(2,3,4$-tri- $O$-benzyl-6-O-tert-butyldiphenylsilyl- $\beta$-D-glucopyranosyl)2,3,4-tri- $O$-benzyl- $\beta$-D-glucopyranosyl|-sn-glycerol (16)

To a solution of compound $\mathbf{1 5}(6.2 \mathrm{~g}, 8.92 \mathrm{mmol})$ in $180 \mathrm{~mL}$ of dry DMF was added benzyl bromide (10 equiv, $10.6 \mathrm{~mL}$ ) at $10^{\circ} \mathrm{C}$. Then $\mathrm{NaH}$ (15 equiv, $2.1 \mathrm{~g}$ ) was added portionwise, and the reaction mixture was stirred for $2 \mathrm{~h}$ at $\mathrm{rt}$. The solvent was evaporated in vacuo; the residue was redissolved in EtOAc and washed two times with saturated $\mathrm{NH}_{4} \mathrm{Cl}$ solution. The organic phase was dried over $\mathrm{MgSO}_{4}$ and the solvent was removed in vacuo. After flash chromatography (petroleum ether/EtOAc 6:1), compound $16(7.28 \mathrm{~g}, 66 \%)$ was obtained. TLC (petroleum ether/EtOAc 4:1): $R_{\mathrm{f}}=0.5 .[\alpha]_{\mathrm{D}}+9.3\left(c 1, \mathrm{CHCl}_{3}\right) .{ }^{1} \mathrm{H}$ NMR $\left(600 \mathrm{MHz}, \mathrm{CDCl}_{3}\right): \delta=1.04(\mathrm{~s}, 9 \mathrm{H}, t-\mathrm{Bu}), 1.30$, $1.35\left(2 \mathrm{~s}, 6 \mathrm{H}, \mathrm{C}\left(\mathrm{CH}_{3}\right)_{2}\right), 3.29(\mathrm{~m}, 1 \mathrm{H}, 5 \mathrm{~b}-\mathrm{H}), 3.42(\mathrm{~m}, 1$ $\left.\mathrm{H}, 1^{\prime} / 3^{\prime}-\mathrm{H}\right), 3.43(\mathrm{~m}, 1 \mathrm{H}, 2 \mathrm{a}-\mathrm{H}), 3.46(\mathrm{~m}, 1 \mathrm{H}, 4 \mathrm{a}-\mathrm{H})$, 3.50 (m, 1 H, 2b-H), 3.57 (m, $1 \mathrm{H}, 5 \mathrm{a}-\mathrm{H}), 3.63(\mathrm{~m}, 1 \mathrm{H}$, 3b-H), 3.64 (m, $\left.1 \mathrm{H}, 1^{\prime} / 3^{\prime}-\mathrm{H}\right), 3.65(\mathrm{~m}, 1 \mathrm{H}, 3 \mathrm{a}-\mathrm{H}), 3.68$ $(\mathrm{m}, 1 \mathrm{H}, 6 \mathrm{a}-\mathrm{H}), 3,75\left(\mathrm{dd}, 1 \mathrm{H}, J_{4,5}=J_{4,3}=9.4 \mathrm{~Hz}, 4 \mathrm{~b}-\right.$ $\mathrm{H}), 3.693 .95\left(\mathrm{~m}, 4 \mathrm{H}, 1^{\prime} / 3^{\prime}-\mathrm{H}, 6 \mathrm{~b}-\mathrm{H}, 1^{\prime} / 3^{\prime}-\mathrm{H}\right), 4.16(\mathrm{~m}$, $\left.1 \mathrm{H}, 2^{\prime}-\mathrm{H}\right), 4.22(\mathrm{~m}, 1 \mathrm{H}, 6 \mathrm{a}-\mathrm{H}), 4.37(\mathrm{~d}, 1 \mathrm{H}$, $\left.J_{1,2}=7.8 \mathrm{~Hz}, 1 \mathrm{a}-\mathrm{H}\right), 4.46\left(\mathrm{~d}, 1 \mathrm{H}, J_{1,2}=7.8 \mathrm{~Hz}, 1 \mathrm{~b}-\mathrm{H}\right)$, $4.514 .55,4.654 .83,4.854 .95,4.975 .03(\mathrm{~m}, 12 \mathrm{H}$, $\left.\mathrm{CH}_{2} \mathrm{Ph}\right), 7.107 .43,7.657 .79(\mathrm{~m}, 40 \mathrm{H}, \mathrm{Ph}) .{ }^{13} \mathrm{C}$ NMR $\left(150.9 \mathrm{MHz}, \mathrm{CDCl}_{3}\right): \delta=19.28\left(1 \mathrm{C}, \mathrm{C}\left(\mathrm{CH}_{3}\right)_{3}\right), 25.35(1$ $\left.\mathrm{C}, \mathrm{C}\left(\mathrm{CH}_{3}\right)_{2}\right), 26.79\left(4 \mathrm{C}, \mathrm{C}\left(\mathrm{CH}_{3}\right)_{2}, \mathrm{C}\left(\mathrm{CH}_{3}\right)_{3}\right), 62.69(1 \mathrm{C}$, C-6b), 66.51 (1 C, C-1'/3'), 68.25 (1 C, C-6a), 70.16 (1 C, C-1'/3'), $74.29\left(1 \mathrm{C}, \mathrm{C}-2^{\prime}\right), 74.8376 .3$ (8 C, CH2Ph, C5a, C-5b), 77.59 (1 C, C-4b), 78.09 (1 C, C-4a), 82.13 (1 C, C-2a), 82.41 (1 C, C-2b), 84.65 (1 C, C-3a), 84.87 (1 C, C-3b), 103.69 (1 C, C-1a), 104.03 (1 C, C-1b), 127.58138 .54 (40 C, Ph). MALDI-MS (positive Mode, Matrix DHB, THF): $[\mathrm{M}+\mathrm{Na}]^{+}, \mathrm{m} / z=1258.6$; found: $\mathrm{m} /$ $z=1257.7,[\mathrm{M}+\mathrm{K}]^{+}, \mathrm{m} / \mathrm{z}=1274.7$; found: $\mathrm{m} / \mathrm{z}=1273.9$. $\mathrm{C}_{76} \mathrm{H}_{86} \mathrm{O}_{13} \mathrm{Si}$ (1235.6): Calcd: C, 73.88; H, 7.02. Found: C, 73.95; H, 7.34.

\subsection{3-O-[6-O-(2,3,4-Tri- $O$-benzyl-6-O-tert-butyldiphe- nylsilyl- $\beta$-D-glucopyranosyl)-2,3,4-tri- $O$-benzyl- $\beta$-D- glucopyranosyl]-sn-glycerol (17)}

To a solution of $16(4 \mathrm{~g}, 3.24 \mathrm{mmol})$ in THF $(100 \mathrm{~mL})$, $200 \mathrm{~mL}$ of $75 \% \mathrm{CH}_{3} \mathrm{COOH}$ in water was added and stir- red for $1.5 \mathrm{~h}$ at $80^{\circ} \mathrm{C}$. The solvent was removed in vacuo and the rest was coevaporated twice with toluene. Purification by flash chromatography (petroleum ether/EtOAc 2:1) gave compound 17 (3.02 g) in a $78 \%$ yield as a colourless foam. TLC (petroleum ether/ EtOAc 2:1): $R_{\mathrm{f}}=0.25 .[\alpha]_{\mathrm{D}}+9.4\left(c 1, \mathrm{CHCl}_{3}\right) .{ }^{1} \mathrm{H}$ NMR $\left(600 \mathrm{MHz}, \mathrm{CDCl}_{3}\right): \delta=1.05(\mathrm{~s}, 9 \mathrm{H}, t$-Bu), 2.0 (br s, $1 \mathrm{H}, \mathrm{OH}), 2.8$ (br s, $1 \mathrm{H}, \mathrm{OH}), 3.313 .35(\mathrm{~m}, 1$ $\mathrm{H}, 5 \mathrm{~b}-\mathrm{H}), 3.383 .45\left(\mathrm{~m}, 3 \mathrm{H}, 1^{\prime} / 3^{\prime}-\mathrm{H}, 4 \mathrm{a}-\mathrm{H}, 2 \mathrm{a}-\mathrm{H}\right)$, $3.50\left(\mathrm{dd}, 1 \mathrm{H}, J_{2,3}=J_{2,1}=8.5 \mathrm{~Hz}, 2 \mathrm{~b}-\mathrm{H}\right), 3.56(\mathrm{~m}, 1$ $\left.\mathrm{H}, 1^{\prime}-\mathrm{H}, 3^{\prime}-\mathrm{H}\right), 3.62,3.65\left(\mathrm{~m}, 2 \mathrm{H}, 1^{\prime} / 3^{\prime}-\mathrm{H}\right), 3.64(\mathrm{~m}, 1$ $\mathrm{H}, 5 \mathrm{a}-\mathrm{H}), 3.65(\mathrm{~m}, 1 \mathrm{H}, 3 \mathrm{~b}-\mathrm{H}), 3.68(\mathrm{~m}, 1 \mathrm{H}, 3 \mathrm{a}-\mathrm{H})$, $3.70(\mathrm{~m}, 1 \mathrm{H}, 6 \mathrm{a}-\mathrm{H}), 3.73\left(\mathrm{~m}, 1 \mathrm{H}, 2^{\prime}-\mathrm{H}\right), 3.74(\mathrm{~m}, 1$ $\mathrm{H}, 4 \mathrm{~b}-\mathrm{H}), 3.92(\mathrm{~m}, 2 \mathrm{H}, 6 \mathrm{~b}-\mathrm{H}), 4.184 .23(\mathrm{~m}, 1 \mathrm{H}$, 6a-H), $4.37\left(\mathrm{~d}, 1 \mathrm{H}, J_{1,2}=7.8 \mathrm{~Hz}, 1 \mathrm{a}-\mathrm{H}\right), 4.48(\mathrm{~d}, 1 \mathrm{H}$, $\left.J_{1,2}=7.8 \mathrm{~Hz}, 1 \mathrm{~b}-\mathrm{H}\right), 4.514 .56,4.654 .99(\mathrm{~m}, 12 \mathrm{H}$, $\left.\mathrm{CH}_{2} \mathrm{Ph}\right), 7.12$ 7.46, 7.66 7.77 (m, 40 H, Ph). MALDIMS (positive Mode, Matrix DHB, THF): $[\mathrm{M}+\mathrm{Na}]^{+}$, $m / z=1218.5$; found: $m / z=1217.2,[\mathrm{M}+\mathrm{K}]^{+}, \mathrm{m} / z=1234.6$; found: $m / z=1234.1 . \quad \mathrm{C}_{73} \mathrm{H}_{82} \mathrm{O}_{13} \mathrm{Si}$ (1195.5): Calcd: $\mathrm{C}$, 73.34; H, 6.91. Found: C, 73.06; H, 6.91.

\subsection{1,2-Di- $O$-myristoyl-3- $O$-[6- $O$-(2,3,4-tri- $O$-benzyl-6- $O$-tert-butyldiphenylsilyl- $\beta$-D-glucopyranosyl)-2,3,4-tri- $O$-benzyl- $\beta$-D-glucopyranosyl]-sn-glycerol (18)}

To a solution of compound $17(3.5 \mathrm{~g}, 2.93 \mathrm{mmol})$ in dry THF (140 mL), $\mathrm{Et}_{3} \mathrm{~N}$ (4.5 mL, 11 equiv) and myristoyl chloride $(4.76 \mathrm{~mL}, 6$ equiv) were added. The reaction mixture was stirred at $50^{\circ} \mathrm{C}$; after $4 \mathrm{~h}$ it was diluted with EtOAc and washed with saturated $\mathrm{NH}_{4} \mathrm{Cl}$ solution. The organic phase was dried over $\mathrm{MgSO}_{4}$, and the solvent was evaporated in vacuo. Purification by flash chromatography (petroleum ether/EtOAc 8:1) yielded compound $18(3.84 \mathrm{~g}, 81 \%)$ as a colourless syrup. TLC (petroleum ether/EtOAc 5:1): $R_{\mathrm{f}}=0.6 \cdot[\alpha]_{\mathrm{D}}+5(c=1$, $\left.\mathrm{CH}_{2} \mathrm{Cl}_{2}\right) .{ }^{1} \mathrm{H}$ NMR $\left(600 \mathrm{MHz}, \mathrm{CDCl}_{3}\right): \delta=0.860 .87$ $(\mathrm{t}, 6 \mathrm{H}, \mathrm{Me}), 1.04(\mathrm{~s}, 9 \mathrm{H}, t$-Bu), $1.091 .38(\mathrm{~s}, 40 \mathrm{H}$, $\mathrm{CH}_{2}$-chain), $1.451 .62\left(\mathrm{~m}, 4 \mathrm{H}, \mathrm{COCH}_{2} \mathrm{CH}_{2} \mathrm{R}\right), 2.12$ $2.28\left(\mathrm{~m}, 4 \mathrm{H}, \mathrm{COCH}_{2} \mathrm{CH}_{2} \mathrm{R}\right), 3.30(\mathrm{~m}, 1 \mathrm{H}, 5 \mathrm{~b}-\mathrm{H})$, $3.41\left(\mathrm{dd}, 1 \mathrm{H}, J_{2,3}=J_{2,1}=8.5 \mathrm{~Hz}, 2 \mathrm{a}-\mathrm{H}\right), 3.47(\mathrm{~m}, 2 \mathrm{H}$, $\left.1^{\prime} / 3^{\prime}-\mathrm{H}, 4 \mathrm{a}-\mathrm{H}\right), 3.50(\mathrm{~m}, 1 \mathrm{H}, 2 \mathrm{~b}-\mathrm{H}), 3.55(\mathrm{~m}, 1 \mathrm{H}, 5 \mathrm{a}-$ $\mathrm{H}), 3.62(\mathrm{~m}, 1 \mathrm{H}, 3 \mathrm{~b}-\mathrm{H}), 3.65(\mathrm{~m}, 1 \mathrm{H}, 6 \mathrm{a}-\mathrm{H}), 3.76$ $\left(\mathrm{dd}, 1 \mathrm{H}, J_{4,5}=J_{4,3}=9.4 \mathrm{~Hz}, 4 \mathrm{a}-\mathrm{H}\right), 3.92(\mathrm{~m}, 1 \mathrm{H}, 6 \mathrm{~b}-$ $\mathrm{H}), 3.96\left(\mathrm{~m}, 1 \mathrm{H}, 1^{\prime} / 3^{\prime}-\mathrm{H}\right), 4.094 .15\left(\mathrm{~m}, 1 \mathrm{H}, 1^{\prime} / 3^{\prime}-\mathrm{H}\right)$, $4.184 .26\left(\mathrm{~m}, 2 \mathrm{H}, 1^{\prime} / 3^{\prime}-\mathrm{H}, 6 \mathrm{a}-\mathrm{H}\right), 4.33(\mathrm{~d}, 1 \mathrm{H}$, $\left.J_{1,2}=7.8 \mathrm{~Hz}, 1 \mathrm{a}-\mathrm{H}\right), \quad 4.42\left(\mathrm{~d}, 1 \mathrm{H}, J_{1,2}=7.8 \mathrm{~Hz}\right.$, 1b-H), 4.50-4.55, 4.64-4.84, 4.86-4.95, 4.97-5.03 (m, 12 $\left.\mathrm{H}, \mathrm{CH}_{2} \mathrm{Ph}\right), 5.13\left(\mathrm{~m}, 1 \mathrm{H}, 2^{\prime}-\mathrm{H}\right), 7.10-7.43,7.64-7.77$ $(\mathrm{m}, 40 \mathrm{H}, \mathrm{Ph}$ ). MALDI-MS (positive Mode, Matrix DHB, THF): $[\mathrm{M}+\mathrm{Na}]^{+}, \quad m / z=1639.2$; found: $\mathrm{m} /$ $z=1638.5, \quad[\mathrm{M}+\mathrm{K}]^{+}, \quad \mathrm{m} / z=1655.3 ;$ found $: \mathrm{m} / z=1654.5$. $\mathrm{C}_{101} \mathrm{H}_{134} \mathrm{O}_{15} \mathrm{Si}$ (1616.2): Calc.: C, 75.06; H, 8.36. Found: C, 74.81; H, 8.67.

5.13. 1,2-Di- $O$-myristoyl-3- $O$-[6- $O$-(2,3,4-tri- $O$-benzyl- $\beta$ D-glucopyranosyl)-2,3,4-tri- $O$-benzyl- $\beta$-D-glucopyranosyl]-sn-glycerol (19)

Compound 18 (3.84 g, $2.38 \mathrm{mmol})$ was dissolved in dry THF $(200 \mathrm{~mL}), \mathrm{CH}_{3} \mathrm{COOH}(0.6 \mathrm{~mL}, 4$ equiv) and TBAF ( $1 \mathrm{M}$ solution, $9.5 \mathrm{~mL}, 4$ equiv) were added and the reaction mixture was stirred for $72 \mathrm{~h}$ at $40{ }^{\circ} \mathrm{C}$. The 
reaction mixture was diluted with AcOEt $(500 \mathrm{~mL})$ and washed with half saturated $\mathrm{NH}_{4} \mathrm{Cl}$ solution $(300 \mathrm{~mL})$, the organic phase was dried over $\mathrm{MgSO}_{4}$ and the solvent was evaporated in vacuo. Purification by flash chromatography (petroleum ether/EtOAc 4:1 $\rightarrow$ 3:1) yielded compound $19(2.48 \mathrm{~g}, 76 \%)$ as a white solid. TLC (petroleum ether/EtOAc 3:1): $R_{\mathrm{f}}=0.45$. Mp $89.4{ }^{\circ} \mathrm{C} .-[\alpha]_{\mathrm{D}}$ +11.9 (c 1, $\left.\mathrm{CHCl}_{3}\right)$. ${ }^{1} \mathrm{H}$ NMR $\left(600 \mathrm{MHz}, \mathrm{CDCl}_{3}\right)$ : $\delta=0.840 .93(\mathrm{t}, 6 \mathrm{H}, \mathrm{Me}), 1.111 .38\left(\mathrm{~s}, 40 \mathrm{H}, \mathrm{CH}_{2^{-}}\right.$ chain), $1.471 .63\left(\mathrm{~m}, 4 \mathrm{H}, \mathrm{COCH}_{2} \mathrm{CH}_{2} \mathrm{R}\right), 2.022 .14$ (s, $1 \mathrm{H}, \mathrm{OH}), 2.142 .29\left(\mathrm{~m}, 4 \mathrm{H}, \mathrm{COCH}_{2} \mathrm{CH}_{2} \mathrm{R}\right), 3.31$ $3.36(\mathrm{~m}, 1 \mathrm{H}, 5 \mathrm{~b}-\mathrm{H}), 3.373 .48(\mathrm{~m}, 3 \mathrm{H}, 2 \mathrm{a}-\mathrm{H}, 2 \mathrm{~b}-\mathrm{H}$, 4a-H), 3.483 .57 (m, 3 H, 5a-H, 4b-H, 1'-H), 3.60-3.73 (m, $4 \mathrm{H}, 3 \mathrm{a}-\mathrm{H}, 3 \mathrm{~b}-\mathrm{H}, 6 \mathrm{a}-\mathrm{H}, 6 \mathrm{~b}-\mathrm{H}), 3.813 .87$ (m, $1 \mathrm{H}$, $6 \mathrm{~b}-\mathrm{H}), 3.92\left(\mathrm{dd}, 1 \mathrm{H}, J_{\text {gem }}=10.9 \mathrm{~Hz}, J_{\text {vic }}=4.5 \mathrm{~Hz}, 1^{\prime}-\right.$ $\mathrm{H}), 4.08\left(\mathrm{dd}, 1 \mathrm{H}, J_{\mathrm{gem}}=11.1 \mathrm{~Hz}, J_{\mathrm{vic}}<1 \mathrm{~Hz}, 6 \mathrm{a}-\mathrm{H}\right)$, $4.114 .17\left(\mathrm{~m}, 1 \mathrm{H}, 3^{\prime}-\mathrm{H}\right), 4.25\left(\mathrm{dd}, 1 \mathrm{H}, J_{\mathrm{gem}}=11.9 \mathrm{~Hz}\right.$, $\left.J_{\text {vic }}=3.3 \mathrm{~Hz}, 3^{\prime}-\mathrm{H}\right), 4.31\left(\mathrm{~d}, 1 \mathrm{H}, J_{1,2}=7.8 \mathrm{~Hz}, 1 \mathrm{a}-\mathrm{H}\right)$, $4.45\left(\mathrm{~d}, 1 \mathrm{H}, J_{1,2}=7.8 \mathrm{~Hz}, 1 \mathrm{~b}-\mathrm{H}\right), 4.514 .56,4.61$ 4.70, $4.734 .87,4.894 .96\left(\mathrm{~m}, 12 \mathrm{H}, \mathrm{CH}_{2} \mathrm{Ph}\right), 5.13$ $5.19\left(\mathrm{~m}, 1 \mathrm{H}, 2^{\prime}-\mathrm{H}\right), 7.137 .37(\mathrm{~m}, 30 \mathrm{H}, \mathrm{Ph}) \cdot{ }^{13} \mathrm{C}$ NMR $\left(150.9 \mathrm{MHz}, \mathrm{CDCl}_{3}\right): \delta=14.1034 .23(26 \mathrm{C}$, $\mathrm{CH}_{2}$-chain), 62.04 (1 C, C-6b), 62.69 (1 C, C-3'), 68.05 (1 C, C-1'), 68.81 (1 C, C-6a), 69.87 (1 C, C-2'), 74.6977.0 (8 C, C-5a, C-5b, $\left.\mathrm{CH}_{2} \mathrm{Ph}\right), 77.58$ (1 C, C-4b), 77.79 (1 C, C-4a), 81.88 (1 C, C-2a), 82.08 (1 C, C2b), 84.50, 84.55 (2 C, C-3a, C-3b), 103.75 (1 C, C-1a), 103.93 (1 C, C-1b), 127.61138 .43 (36C, Ph), 172.97, 173.29 ( $2 \mathrm{C}, \mathrm{COCH}_{2} \mathrm{CH}_{2} \mathrm{R}$ ). MALDI-MS (positive Mode, Matrix DHB, THF): $[\mathrm{M}+\mathrm{Na}]^{+}, \mathrm{m} / z=1400.8$; found: $m / z=1399.9,[\mathrm{M}+\mathrm{K}]^{+}, \mathrm{m} / \mathrm{z}=1416.9$; found: $\mathrm{m} /$ $z=1417.2 . \mathrm{C}_{85} \mathrm{H}_{116} \mathrm{O}_{15}$ (1377.8): Calcd: $\mathrm{C}, 74.10 ; \mathrm{H}$, 8.49. Found: $\mathrm{C}, 74.06 ; \mathrm{H}, 8.61$.

5.14. [Benzyloxy]-[diisopropylamino]-[1,2-di- $O$-myristoyl3-O-\{6-O-(2,3,4-tri-O-benzyl- $\beta$-D-glucopyranosyl)-2,3,4tri- $O$-benzyl- $\beta$-D-glucopyranosyl\}-sn-glycero|phosphane (3)

Compound $19(0.7 \mathrm{~g}, 0.508 \mathrm{mmol})$ was dried for $1 \mathrm{~h}$ together with tetrazole $(21.4 \mathrm{mg}, 0.6 \mathrm{eq})$ in high vacuum. Under argon atmosphere benzyloxybis-(diisopropylamino)-phosphane (215 mg, 1.3 equiv) dissolved in $10 \mathrm{~mL}$ of dry $\mathrm{CH}_{2} \mathrm{Cl}_{2}$ was added. After $30 \mathrm{~min}$ of stirring at $\mathrm{rt}$ the tetrazole was dissolved, the reaction mixture was stirred for $1.5 \mathrm{~h}$; then the mixture was diluted with $\mathrm{CH}_{2} \mathrm{Cl}_{2}$ and poured over a saturated $\mathrm{NaHCO}_{3}$ solution. The organic phase was dried over $\mathrm{MgSO}_{4}$ and removed in vacuo below $30^{\circ} \mathrm{C}$. Fast purification over flash silica gel (petroleum ether/EtOAc 5:1, $+1 \% \mathrm{NEt}_{3}$ ) yielded compound $3(650 \mathrm{mg}, 79 \%)$ after one co-evaporation with toluene as a colourless syrup. TLC (petroleum ether/EtOAc 5:1, +1\% NEt $\left.{ }_{3}\right): R_{\mathrm{f}}=0.9 .[\alpha]_{\mathrm{D}}+7.5(c 1$, $\left.\mathrm{CHCl}_{3}\right) .{ }^{1} \mathrm{H}$ NMR $\left(600 \mathrm{MHz}, \mathrm{CDCl}_{3}\right): \delta=0.800 .92$ (t, $6 \mathrm{H}, \mathrm{Me}$ ), 1.061 .38 (s, $40 \mathrm{H}, \mathrm{CH}_{2}$-chain), 1.451 .62 $\left(\mathrm{m}, 4 \mathrm{H}, \quad \mathrm{COCH}_{2} \mathrm{CH}_{2} \mathrm{R}\right), 2.102 .58(\mathrm{~m}, 4 \mathrm{H}$, $\left.\mathrm{COCH}_{2} \mathrm{CH}_{2} \mathrm{R}\right), 3.38(\mathrm{~m}, 1 \mathrm{H}, 2 \mathrm{a}-\mathrm{H}), 3.39(\mathrm{~m}, 1 \mathrm{H}, 5 \mathrm{~b}-$ $\mathrm{H}), 3.41(\mathrm{~m}, 1 \mathrm{H}, 4 \mathrm{a}-\mathrm{H}), 3.42(\mathrm{~m}, 1 \mathrm{H}, 2 \mathrm{~b}-\mathrm{H}), 3.43(\mathrm{~m}$, $\left.1 \mathrm{H}, 3^{\prime}-\mathrm{H}\right), 3.51(\mathrm{~m}, 1 \mathrm{H}, 5 \mathrm{a}-\mathrm{H}), 3.54 / 3.59(\mathrm{~m}, 1 \mathrm{H}, 4 \mathrm{~b}-$ $\mathrm{H}), 3.61(\mathrm{~m}, 2 \mathrm{H}, 3 \mathrm{a}-\mathrm{H}, 3 \mathrm{~b}-\mathrm{H}), 3.65(\mathrm{~m}, 3 \mathrm{H}$, $\left.\mathrm{NCH}\left(\mathrm{CH}_{3}\right)_{2}, 6 \mathrm{a}-\mathrm{H}\right), 3.79,3.89,4.00(\mathrm{~m}, 2 \mathrm{H}, 6 \mathrm{~b}-\mathrm{H})$, $3.92\left(\mathrm{~m}, 1 \mathrm{H}, 3^{\prime}-\mathrm{H}\right), 4.074 .24\left(\mathrm{~m}, 3 \mathrm{H}, 6 \mathrm{a}-\mathrm{H}, 1^{\prime}-\mathrm{H}\right)$, $4.28\left(\mathrm{~d}, 1 \mathrm{H}, J_{1,2}=7.8 \mathrm{~Hz}, 1 \mathrm{a}-\mathrm{H}\right), 4.43(\mathrm{~d}, 1 \mathrm{H}$,
$\left.J_{1,2}=7.8 \mathrm{~Hz}, \quad 1 \mathrm{~b}-\mathrm{H}\right), 4.454 .52,4.624 .84,4.844 .97$ $\left(\mathrm{m}, 14 \mathrm{H}, \mathrm{CH}_{2} \mathrm{Ph}\right), 5.085 .15\left(\mathrm{~m}, 1 \mathrm{H}, 2^{\prime}-\mathrm{H}\right), 7.067 .44$ $(\mathrm{m}, \quad 35 \mathrm{H}, \mathrm{Ph}) .{ }^{13} \mathrm{C}$ NMR $\left(150.9 \mathrm{MHz}, \mathrm{CDCl}_{3}\right)$ : $\delta=14.1134 .23\left(30 \mathrm{C}, 2\left(\mathrm{NCH}\left(\mathrm{CH}_{3}\right)_{2}\right), \mathrm{CH}_{2}\right.$-chain $)$, 43.1, $43.18\left(2 \mathrm{C}, 2\left(\mathrm{NCH}\left(\mathrm{CH}_{3}\right)_{2}\right)\right), 62.46,62.70(2 \mathrm{C}$, C-1', C-6b), 65.2165 .39 (1 C, $\left.\mathrm{POC}_{2} \mathrm{Ph}\right), 67.99$ (1 C, C-3'), 68.41 (1 C, C-6a), 69.81 (1 C, C-2'), 74.6677 .0 (8 C, C-5a, C-5b, $\left.\mathrm{CH}_{2} \mathrm{Ph}\right), 77.74 / 77.85$ (1 C, C-4b), 78.05 (1 C, C-4a), 81.9182 .15 (2 C, C-2a, C-2b), 84.5184 .78 (2 C, C-3a, C-3b), 103.65 (1 C, C-1a), 103.92103 .98 (1 C, C-1b), 126.84138 .61 (42 C, Ph), $172.88, \quad 173.24 \quad\left(2 \mathrm{C}, \mathrm{COCH}_{2} \mathrm{CH}_{2} \mathrm{R}\right) .{ }^{31} \mathrm{P} \quad \mathrm{NMR}$ $\left(242.9 \mathrm{MHz}, \mathrm{CDCl}_{3}\right): \delta=149.79,149.89$ (2s, $\left.1 \mathrm{P}\right)$. $\mathrm{C}_{98} \mathrm{H}_{136} \mathrm{NO}_{16} \mathrm{P}$ (1615.1): Calcd: $\mathrm{C}, 72.88 ; \mathrm{H}, 8.49 ; \mathrm{N}$, 0.87. Found: C, 72.61; H, 8.69; N, 0.88 .

\subsection{Hexaphosphate 21}

Compound $20^{13}$ (494 mg, $0.202 \mathrm{mmol}$ ) and phosphite amide 3 (424 mg, 1.3 equiv) were coevaporated each with dry $\mathrm{CH}_{2} \mathrm{Cl}_{2}(10 \mathrm{~mL})$ and dried for $1 \mathrm{~h}$ in high vacuum. Compound 3 was dissolved in $15 \mathrm{~mL}$ of dry $\mathrm{CH}_{2} \mathrm{Cl}_{2}$ and was added, under argon atmosphere, to compound 20; tetrazole ( $29 \mathrm{mg}, 2$ equiv, dried previously for $1 \mathrm{~h}$ in high vacuum) was also added. The reaction mixture was stirred at $\mathrm{rt}$ under argon atmosphere. After $70 \mathrm{~min}, t-\mathrm{BuO}_{2} \mathrm{H}(0.6 \mathrm{~mL})$ was added dropwise and the reaction mixture was stirred for another $35 \mathrm{~min} \mathrm{CH}_{2} \mathrm{Cl}_{2}$ was added and the mixture was washed with saturated $\mathrm{NaHCO}_{3}$ solution, the organic phase was dried over $\mathrm{MgSO}_{4}$ and the solvent was removed in vacuo. Purification by flash chromatography (toluene/acetone 1:1) gave compound $21(602 \mathrm{mg}, 75 \%)$ as a colourless syrup, which was stored at $-20^{\circ} \mathrm{C}$. TLC (toluene/acetone 1:1): $R_{\mathrm{f}}=0.35, R_{\mathrm{f}}=0.42 .[\alpha]_{\mathrm{D}}+12.1\left(c 1, \mathrm{CHCl}_{3}\right) .{ }^{1} \mathrm{H}$ NMR (600 MHz, $\left.\mathrm{CDCl}_{3}\right): \delta=0.810 .92(\mathrm{t}, 6 \mathrm{H}, \mathrm{Me})$, $1.111 .35\left(\mathrm{~m}, 40 \mathrm{H}, \mathrm{CH}_{2}\right.$-chain), $1.441 .61(\mathrm{~m}, 4 \mathrm{H}$, $\mathrm{COCH}_{2} \mathrm{CH}_{2} \mathrm{R}$ ), 1.841 .98 (m, $\left.3 \mathrm{H}, \mathrm{NHAc}\right), 2.092 .25$ $\left(\mathrm{m}, 4 \mathrm{H}, \mathrm{COCH}_{2} \mathrm{CH}_{2} \mathrm{R}\right), 3.32(\mathrm{~m}, 1 \mathrm{H}, 5 \mathrm{a} / \mathrm{b}-\mathrm{H}), 3.33$ (m, $1 \mathrm{H}, 2 \mathrm{a}-\mathrm{H}), 3,35\left(\mathrm{~m}, 1 \mathrm{H}, 1^{\prime}-\mathrm{H}\right), 3.37(\mathrm{~m}, 1 \mathrm{H}, 5 \mathrm{a} /$ b-H), $3.38(\mathrm{~m}, 1 \mathrm{H}, 2 \mathrm{~b}-\mathrm{H}), 3.43,3.44(\mathrm{~m}, 2 \mathrm{H}, 4 \mathrm{a}-\mathrm{H}$, 4b-H), 3.49 (m, $2 \mathrm{H}, 18-\mathrm{H}), 3.57(\mathrm{~m}, 2 \mathrm{H}, 3 \mathrm{a}-\mathrm{H}, 3 \mathrm{~b}-$ H), 3.58-3.60 (m, $3 \mathrm{H}, 6 \mathrm{c}-\mathrm{H}, 6 \mathrm{a}-\mathrm{H}), 3.63(\mathrm{~m}, 4 \mathrm{H}, 5-\mathrm{H}$, 8-H, 11-H, 14-H), $3.64(\mathrm{~m}, 1 \mathrm{H}, 4 \mathrm{c}-\mathrm{H}), 3.67(\mathrm{~m}, 12 \mathrm{H}$, $\mathrm{OMe}), 3.68(\mathrm{~m}, 1 \mathrm{H}, 3 \mathrm{c}-\mathrm{H}), 3.69(\mathrm{~m}, 1 \mathrm{H}, 2-\mathrm{H}), 3.72$ (m, $1 \mathrm{H}, 17-\mathrm{H}), 3.75(\mathrm{~m}, 1 \mathrm{H}, 5 \mathrm{c}-\mathrm{H}), 3.83\left(\mathrm{~m}, 1 \mathrm{H}, 1^{\prime}-\right.$ H), 3.96, $4.04(\mathrm{~m}, 18 \mathrm{H}, 3-\mathrm{H}, 4-\mathrm{H}, 6-\mathrm{H}, 7-\mathrm{H}, 9-\mathrm{H}, 10-$ $\mathrm{H}, 12-\mathrm{H}, 13-\mathrm{H}, 15-\mathrm{H}), 4.06,4.15\left(\mathrm{~m}, 2 \mathrm{H}, 3^{\prime}-\mathrm{H}\right), 4.08$ $(\mathrm{m}, 1 \mathrm{H}, 6 \mathrm{a}-\mathrm{H}), 4.07,4.17(\mathrm{~m}, 4 \mathrm{H}, 16-\mathrm{H}, 6 \mathrm{~b}-\mathrm{H}), 4.20$ $(\mathrm{m}, 1 \mathrm{H}, 1 \mathrm{a}-\mathrm{H}), 4.35(\mathrm{~m}, 1 \mathrm{H}, 2 \mathrm{c}-\mathrm{H}), 4.41(\mathrm{~m}, 1 \mathrm{H}, 1 \mathrm{~b}-$ $\mathrm{H}), 4.74(\mathrm{~m}, 1 \mathrm{H}, 1 \mathrm{c}-\mathrm{H}), 4.294 .91\left(\mathrm{~m}, 30 \mathrm{H}, \mathrm{CH}_{2} \mathrm{Ph}\right)$, $4.93\left(\mathrm{~m}, 12 \mathrm{H}, \mathrm{POCH}_{2} \mathrm{Ph}\right), 5.06\left(\mathrm{~m}, 1 \mathrm{H}, 2^{\prime}-\mathrm{H}\right), 6.69$ $6.80\left(\mathrm{~m}, 8 \mathrm{H}, \mathrm{Ph}_{\mathrm{MPM}}\right), 7.037 .34(\mathrm{~m}, 93 \mathrm{H}, \mathrm{Ph}) .{ }^{13} \mathrm{C}$ NMR (150.9 MHz, $\left.\mathrm{CDCl}_{3}\right): \delta=14.11(2 \mathrm{C}, \mathrm{Me}), 23.1$ (1 C, NHAc), 24.84 ( $\left.2 \mathrm{C}, \mathrm{COCH}_{2} \mathrm{CH}_{2} \mathrm{R}\right), 22.68 / 29.14$ 31.91 (20 C, $\mathrm{CH}_{2}$-chain), 34.04, $34.22\left(2 \mathrm{C}, \mathrm{COCH}_{2} \mathrm{R}\right)$, 52.8 (1 C, C-2c), 55.17 (4 C, OMe), $63.2\left(1 \mathrm{C}, \mathrm{C}-3^{\prime}\right)$, $65.567 .2\left(12 \mathrm{C}, \mathrm{CH}_{2-\text { Glyc }}, \mathrm{C}-6 \mathrm{~b}\right), 68.1$ (1 C, C-1'), 68.5 (1 C, C-6c), 68.6 (1 C, C-6a), 69.0 (1 C, C-18), 69.5 (6 C, $\left.\mathrm{POCH}_{2} \mathrm{Ph}\right), 69.8\left(1 \mathrm{C}, \mathrm{C}-2^{\prime}\right), 72$ (1 C, C-5c), 73.5 (1 C, C-5a/b), 75 (1 C, C-4a/b), 75.4 (4 C, C-5, C-8, C11, C-14), 72-77.0 (15 C, $\left.\mathrm{CH}_{2} \mathrm{Ph}\right), 76.8$ (1 C, C-17), 77.5 (1 C, C-4c), 78.2 (1 C, C-2), 81.2 (1 C, C-3c), 81.8 
(2 C, C-2a, C-2b), 84.6 (2 C, C-3a, C-3b), 100.05 (1 C, C1c), 103.1 (1 C, C-1a), 103.6 (1 C, C-1b). MALDI-MS (positive Mode, Matrix $p$-nitroaniline+NaI, THF): $[\mathrm{M}+\mathrm{Na}]^{+}, \quad m / z=4002.3 ; \quad$ found: $\quad m / z=4000.0$. $\mathrm{C}_{220} \mathrm{H}_{271} \mathrm{NO}_{54} \mathrm{P}_{6}$ (3979.3): Calcd: $\mathrm{C}, 66.40 ; \mathrm{H}, 6.86 ; \mathrm{N}$, 0.35 . Found: C, 66.40; H, 7.09; N, 0.36.

\subsection{Hexaphosphate 22}

Compound 21 (417 mg, $0.105 \mathrm{mmol})$ was dissolved in acetonitrile/toluene/water $(60: 3: 4,20 \mathrm{~mL})$ and cooled to $-10{ }^{\circ} \mathrm{C} . \mathrm{Ce}\left(\mathrm{NH}_{4}\right)_{2}\left(\mathrm{NO}_{3}\right)_{6}(1.15 \mathrm{~g}, 20$ equiv) was added portionwise and the reaction mixture was stirred for 20 min at $-10{ }^{\circ} \mathrm{C}$, the cooling bath was removed and the reaction mixture was stirred for another $3040 \mathrm{~min}$ (TLC-monitoring). After this time, the reaction mixture was diluted with EtOAc and washed with saturated $\mathrm{NaHCO}_{3}$ solution, the organic phase was dried over $\mathrm{MgSO}_{4}$ and evaporated in vacuo. Fast purification on silica gel (toluene/acetone $1: 1 \rightarrow 1: 3$ ) yielded compound $22(246 \mathrm{mg}, 67 \%)$ as a colourless syrup, which was stored at $-20^{\circ} \mathrm{C}$. TLC (toluene/acetone 1:1): $R_{\mathrm{f}}=0.40$, $R_{\mathrm{f}}=0.45 . \quad[\alpha]_{\mathrm{D}}+13.6 \quad\left(\begin{array}{lllll}c & 0.5, & \left.\mathrm{CHCl}_{3}\right) . & { }^{1} \mathrm{H} & \mathrm{NMR}\end{array}\right.$ $\left(600 \mathrm{MHz}, \mathrm{CDCl}_{3}\right): \delta=0.820 .94(\mathrm{t}, 6 \mathrm{H}, \mathrm{Me}), 1.09$ $1.38\left(\mathrm{~m}, 40 \mathrm{H}, \mathrm{CH}_{2}\right.$-chain), $1.441 .59(\mathrm{~m}, 4 \mathrm{H}$, $\mathrm{COCH}_{2} \mathrm{CH}_{2} \mathrm{R}$ ), 1.851 .99 (m, $3 \mathrm{H}, \mathrm{NHAc}$ ), 2.092 .26 (m, $\left.4 \mathrm{H}, \mathrm{COCH}_{2} \mathrm{CH}_{2} \mathrm{R}\right), 3.33(\mathrm{~m}, 2 \mathrm{H}, 2 \mathrm{a}-\mathrm{H}, 5 \mathrm{a} / \mathrm{b}-\mathrm{H})$, $3.37(\mathrm{~m}, 1 \mathrm{H}, 2 \mathrm{~b}-\mathrm{H}), 3,38\left(\mathrm{~m}, 1 \mathrm{H}, 1^{\prime}-\mathrm{H}\right), 3.39(\mathrm{~m}, 1 \mathrm{H}$, 5a/b-H), 3.45 (m, 2 H, 4a-H, 4b-H), 3.52 (m, 2 H, 18H), 3.57 (m, $2 \mathrm{H}, 3 \mathrm{a}-\mathrm{H}, 3 \mathrm{~b}-\mathrm{H}), 3.59(\mathrm{~m}, 3 \mathrm{H}, 6 \mathrm{c}-\mathrm{H}, 6 \mathrm{a}-$ $\mathrm{H}), 3.67(\mathrm{~m}, 1 \mathrm{H}, 4 \mathrm{c}-\mathrm{H}), 3.68(\mathrm{~m}, 1 \mathrm{H}, 3 \mathrm{c}-\mathrm{H}), 3.73(\mathrm{~m}$, $2 \mathrm{H}, 2-\mathrm{H}, 17-\mathrm{H}), 3.78(\mathrm{~m}, 1 \mathrm{H}, 5 \mathrm{c}-\mathrm{H}), 3.85\left(\mathrm{~m}, 1 \mathrm{H}, 1^{\prime}-\right.$ $\mathrm{H}), 3.96,4.07$ (m, $\left.\mathrm{CH}_{2-\mathrm{Glyc}}\right), 4.05(\mathrm{~m}, 4 \mathrm{H}, 1-\mathrm{H}, 3-\mathrm{H})$, 4.08, 4.16 (m, 2 H, 3'-H), 4.004 .26 (m, 4 H, 6b-H, 16$\mathrm{H}), 4.21(\mathrm{~m}, 1 \mathrm{H}, 1 \mathrm{a}-\mathrm{H}), 4.31(\mathrm{~m}, 1 \mathrm{H}, 2 \mathrm{c}-\mathrm{H}), 4.344 .92$ $\left(\mathrm{m}, 22 \mathrm{H}, \mathrm{CH}_{2} \mathrm{Ph}\right), 4.42(\mathrm{~m}, 1 \mathrm{H}, 1 \mathrm{~b}-\mathrm{H}), 4.82(\mathrm{~m}, 1 \mathrm{H}$, $1 \mathrm{c}-\mathrm{H}), 5.01\left(\mathrm{~m}, 12 \mathrm{H}, \mathrm{POCH} \mathrm{H}_{2} \mathrm{Ph}\right), 5.07\left(\mathrm{~m}, 1 \mathrm{H}, 2^{\prime}-\mathrm{H}\right)$, $7.047 .46(\mathrm{~m}, 85 \mathrm{H}, \mathrm{Ph}) .{ }^{13} \mathrm{C}$ NMR $\left(150.9 \mathrm{MHz}, \mathrm{CDCl}_{3}\right)$ : $\delta=14.1(2 \mathrm{C}, \mathrm{Me}), 23.0$ (1 C, NHAc), 24.9 (2 C, $\left.\mathrm{COCH}_{2} \mathrm{CH}_{2} \mathrm{R}\right), 22.7 / 29.129 .7 / 31.9\left(20 \mathrm{C}, \mathrm{CH}_{2}\right.$-chain), 34.0, $34.2\left(2 \mathrm{C}, \mathrm{COCH}_{2} \mathrm{R}\right), 52,8$ (1 C, C-2c), 62.9 (1 C, C-3'), 65.8 (2 C, C-1, C-3), 66.0-68.2 (1'-C, $\mathrm{CH}_{2 \text {-Glyc' }}{ }^{-}$ $\mathrm{OH}, \mathrm{C}-6 \mathrm{~b}, \mathrm{C}-16), 68.5$ (1 C, C-6c), 69.0 (1 C, C-18), $69.8\left(6 \mathrm{C}, \mathrm{POCH}{ }_{2} \mathrm{Ph}\right), 69.9\left(1 \mathrm{C}, \mathrm{C}-2^{\prime}\right), 71.8(1 \mathrm{C}, \mathrm{C}-$ 5c), $72.276 .0\left(11 \mathrm{C}, \mathrm{CH}_{2} \mathrm{Ph}\right), 73.8(1 \mathrm{C}, \mathrm{C}-5 \mathrm{a} / \mathrm{b}), 75.2$ (1 C, C-4a/b), 76.6 (2 C, C-2, C-17), 77.1 (1 C, C-4a/b), 78.0 (1 C, C-4c), 78.1 (1 C, C-5a/b), 81.0 (1 C, C-3c), 81.9 (2 C, C-2a, C-2b), 84.5 (2 C, C-3a, C-3b), 99.8 (1 C, C-1c), 103.9 (1 C, C-1a), 104.1 (1 C, C-1b). FABMS (positive Mode): $[\mathrm{M}+\mathrm{Na}]^{+}, \quad m / z=3519.7$; gef.: $m / z=3522$. MALDI-MS (positive Mode, Matrix $p$-nitroaniline+NaI, THF): $[\mathrm{M}+\mathrm{Na}]^{+}, m / z=3519.7$; found: $m / z=3518 . \quad \mathrm{C}_{188} \mathrm{H}_{237} \mathrm{NO}_{50} \mathrm{P}_{6} \mathrm{H}_{2} \mathrm{O}$ (3514.7): Calcd: $\mathrm{C}$, 64.25; H, 6.85; N, 0.40. Found: C, 64.33; H, 7.31; N, 0.40 .

\subsection{Hexaphosphate 23a}

Compound 22 (197 mg, $0.056 \mathrm{mmol}$ ), PyBOP (585 mg, 20 equiv) and Z-D-Ala triethylammonium salt $(365 \mathrm{mg}$, 20 equiv) were dried separately for $3 \mathrm{~h}$ in high vacuum. After this time, 22 was dissolved in dry $\mathrm{CH}_{2} \mathrm{Cl}_{2}(15 \mathrm{~mL})$, Z-D-Ala triethylammonium salt and PyBOP were add- ed. $N$-Methyl imidazole ( $180 \mu \mathrm{L}, 40$ equiv) was added dropwise and the reaction mixture was stirred for 2.5 $3 \mathrm{~h}$ at $\mathrm{rt}$ under argon atmosphere. The reaction mixture was diluted with $\mathrm{CH}_{2} \mathrm{Cl}_{2}$ and washed with saturated $\mathrm{NH}_{4} \mathrm{Cl}$ solution. The organic phase was dried over $\mathrm{MgSO}_{4}$ and the solvent was removed in vacuo. Purification by flash chromatography (toluene/acetone 3:1) and second column (toluene/acetone 3:1) yielded 23a as a mixture of diastereomers $(170 \mathrm{mg}, 70 \%)$ as a colourless syrup which was stored at $-20^{\circ} \mathrm{C}$. TLC (toluene/acetone 1:1): $R_{\mathrm{f}}=0.71, R_{\mathrm{f}}=0.75 .[\alpha]_{\mathrm{D}}+15\left(c 0.15, \mathrm{CHCl}_{3}\right)$. ${ }^{1} \mathrm{H}$ NMR $\left(600 \mathrm{MHz}, \mathrm{CDCl}_{3}\right): \delta=0.88(\mathrm{t}, 6 \mathrm{H}, \mathrm{Me})$, $1.121 .41\left(\mathrm{~m}, 52 \mathrm{H}, \mathrm{CH}_{2}\right.$-chain, Ala-Me), 1.461 .59 $\left(\mathrm{m}, 4 \mathrm{H}, \mathrm{COCH}_{2} \mathrm{CH}_{2} \mathrm{R}\right), 1.861 .99(\mathrm{~m}, 3 \mathrm{H}, \mathrm{NHAc})$, $2.102 .26\left(\mathrm{~m}, 4 \mathrm{H}, \mathrm{COCH}_{2} \mathrm{CH}_{2} \mathrm{R}\right), 3.33(\mathrm{~m}, 1 \mathrm{H}, 5 \mathrm{a} / \mathrm{b}-$ $\mathrm{H}), 3.34(\mathrm{~m}, 1 \mathrm{H}, 2 \mathrm{a}-\mathrm{H}), 3.35(\mathrm{~m}, 1 \mathrm{H}, 5 \mathrm{a} / \mathrm{b}-\mathrm{H}), 3.36$ (m, $\left.1 \mathrm{H}, 1^{\prime}-\mathrm{H}\right), 3.39(\mathrm{~m}, 1 \mathrm{H}, 2 \mathrm{~b}-\mathrm{H}), 3.44,3.46(\mathrm{~m}, 2$ $\mathrm{H}, 4 \mathrm{a}-\mathrm{H}, 4 \mathrm{~b}-\mathrm{H}), 3.52(\mathrm{~m}, 2 \mathrm{H}, 18-\mathrm{H}), 3.57(\mathrm{~m}, 2 \mathrm{H}$, 3a-H, 3b-H), 3.59 (m, $2 \mathrm{H}, 6 \mathrm{c}-\mathrm{H}), 3.67(\mathrm{~m}, 1 \mathrm{H}, 6 \mathrm{a}-\mathrm{H})$, $3.69(\mathrm{~m}, 2 \mathrm{H}, 4 \mathrm{c}-\mathrm{H}, 3 \mathrm{c}-\mathrm{H}), 3.74(\mathrm{~m}, 2 \mathrm{H}, 2-\mathrm{H}, 17-\mathrm{H})$, $3.76(\mathrm{~m}, 1 \mathrm{H}, 5 \mathrm{c}-\mathrm{H}), 3.85\left(\mathrm{~m}, 1 \mathrm{H}, 1^{\prime}-\mathrm{H}\right), 4.01(\mathrm{~m}, 20$ $\mathrm{H}, 1-\mathrm{H}, 3-\mathrm{H}, 4-\mathrm{H}, 6-\mathrm{H}, 7-\mathrm{H}, 9-\mathrm{H}, 10-\mathrm{H}, 12-\mathrm{H}, 13-\mathrm{H}$, 15-H), $4.07(\mathrm{~m}, 1 \mathrm{H}, 16-\mathrm{H}), 4.08\left(\mathrm{~m}, 1 \mathrm{H}, 3^{\prime}-\mathrm{H}\right), 4.10$ (m, $1 \mathrm{H}, 6 \mathrm{a}-\mathrm{H}), 4.16\left(\mathrm{~m}, 1 \mathrm{H}, 3^{\prime}-\mathrm{H}\right), 4.18(\mathrm{~m}, 3 \mathrm{H}, 6 \mathrm{~b}-$ $\mathrm{H}, 16-\mathrm{H}), 4.21(\mathrm{~m}, 1 \mathrm{H}, 1 \mathrm{a}-\mathrm{H}), 4.33(\mathrm{~m}, 4 \mathrm{H}$, CHNHCbz), $4.34(\mathrm{~m}, 1 \mathrm{H}, 2 \mathrm{c}-\mathrm{H}), 4.32-4.92(\mathrm{~m}, 22 \mathrm{H}$, $\left.\mathrm{CH}_{2} \mathrm{Ph}\right), 4.43(\mathrm{~m}, 1 \mathrm{H}, 1 \mathrm{~b}-\mathrm{H}), 4.79(\mathrm{~m}, 1 \mathrm{H}, 1 \mathrm{c}-\mathrm{H})$, $4.98\left(\mathrm{~m}, 4 \mathrm{H}, \mathrm{CH}_{2} \mathrm{Cbz}\right), 4.99\left(\mathrm{~m}, 12 \mathrm{H}, \mathrm{POCH}_{2} \mathrm{Ph}\right)$, $5.05\left(\mathrm{~m}, 4 \mathrm{H}, \mathrm{CH}_{2} \mathrm{Cbz}\right), 5.08\left(\mathrm{~m}, 1 \mathrm{H}, 2^{\prime}-\mathrm{H}\right), 5.09(\mathrm{~m}$, $4 \mathrm{H}, 5,8,11,14-\mathrm{H}), 5.566 .14(\mathrm{NH}), 7.047 .46(\mathrm{~m}$, $105 \mathrm{H}, \mathrm{Ph}) .{ }^{13} \mathrm{C}$ NMR $\left(150.9 \mathrm{MHz}, \mathrm{CDCl}_{3}\right): \delta=14.4$ (2 C, Me), 18.3 (4 C, Ala-Me), 23.1 (1 C, NHAc), 25.2 (2 $\left.\mathrm{C}, \mathrm{COCH}_{2} \mathrm{CH}_{2} \mathrm{R}\right), 29.8$ (2 $0 \mathrm{C}, \mathrm{CH}_{2}$-chain), 34.3, $34.5\left(2 \mathrm{C}, \mathrm{COCH}_{2} \mathrm{R}\right), 49.9$ (4 C, CHNHCbz), 53.0 (1 $\mathrm{C}, \mathrm{C}-2 \mathrm{c}), 63.0\left(1 \mathrm{C}, \mathrm{C}-3^{\prime}\right), 65.1$ (10 C, C-1, C-3, C-4, C-6, C-7, C-9, C-10, C-12, C-13, C-15), 66.9 (1 C, C6b), 67.0 (4 C, $\left.\mathrm{CH}_{2} \mathrm{Cbz}\right), 67.5$ (1 C, C-16), 68.3 (1 C, C-1'), 68.9 (2 C, C-6a, C-6c), 69.1 (1 C, C-18), 70.0 (1 $\left.\mathrm{C}, \mathrm{C}-2^{\prime}\right), 70.1\left(6 \mathrm{C}, \mathrm{POCH} \mathrm{H}_{2} \mathrm{Ph}\right), 71.0$ (4 C, CH-Ala), $72.0(1 \mathrm{C}, \mathrm{C}-5 \mathrm{c}), 72.2-76.0\left(11 \mathrm{C}, \mathrm{CH}_{2} \mathrm{Ph}\right), 73.8(1 \mathrm{C}$, C-5a/b), 75.3 (1 C, C-4a/b), 76.8 (2 C, C-2, C-17), 77.1 (1 C, C-4a/b), 77.9 (1 C, C-4c), 78.0 (1 C, C-5a/b), 81.1 (1 C, C-3c), 82.1 (2 C, C-2a, C-2b), 84.8 (2 C, C3a, C-3b), 100.2 (1 C, C-1c), 103.8 (1 C, C-1a), 104.1 (1 C, C-1b). MALDI-MS (positive Mode, Matrix $p$-nitroaniline+NaI, $\mathrm{MeOH}):[\mathrm{M}+\mathrm{Na}]^{+}, \mathrm{m} / z=4340$; found: $m / z=4336 . \quad \mathrm{C}_{232} \mathrm{H}_{281} \mathrm{~N}_{5} \mathrm{O}_{62} \mathrm{P}_{6} \quad$ (4317.6): Calcd: $\mathrm{C}$, 64.54; H, 6.56; N, 1.62. Found: C, 64.34; H, 6.75; N, 1.51 .

\subsection{Target molecule 1a}

The diastereomers 23a ( $83 \mathrm{mg}, 0.019 \mathrm{mmol})$ were dissolved in $\mathrm{CH}_{2} \mathrm{Cl}_{2} / \mathrm{MeOH} / \mathrm{H}_{2} \mathrm{O}(7.5: 7.5: 1.5,6 \mathrm{~mL})$, treated with Pearlman's catalyst (10\% in weight) and under hydrogen atmosphere, with a $\mathrm{H}_{2}$-filled balloon, was stirred overnight at $\mathrm{rt}$. The reaction mixture was filtered through Celite, washed with $\mathrm{CH}_{2} \mathrm{Cl}_{2} / \mathrm{MeOH} / \mathrm{H}_{2} \mathrm{O}$ (7.5:7.5:1.5, $2 \mathrm{~mL}$ ) and the filtrate was diluted with $0.1 \mathrm{M} \mathrm{NH}_{4} \mathrm{OAc}$-buffer ( $\mathrm{pH}$ 4.8). The solvent was lyophilized and purified using hydrophobic interaction HPLC on octylsepharose. After lyophilization, compound 1a (20 mg, 47\%) was obtained as white powder. $[\alpha]_{\mathrm{D}}+6.9$ 
$\left(c 0.13, \mathrm{H}_{2} \mathrm{O}\right) .{ }^{1} \mathrm{H} \mathrm{NMR}\left(600 \mathrm{MHz}, \mathrm{D}_{2} \mathrm{O}\right): \delta=0.820 .94$ (m, $6 \mathrm{H}, \mathrm{Me}), 1.161 .43$ (m, $40 \mathrm{H}, \mathrm{CH}_{2}$-chain), 1.551 .69 (m, $16 \mathrm{H}$, Ala-Me, $\mathrm{COCH}_{2} \mathrm{CH}_{2} \mathrm{R}$ ), 2.11 (s, $3 \mathrm{H}, \mathrm{NHAc}$ ), $2.292 .47\left(\mathrm{~m}, 4 \mathrm{H}, \mathrm{COCH}_{2} \mathrm{CH}_{2} \mathrm{R}\right), 3.274 .59(\mathrm{~m}, 54 \mathrm{H})$, 5.10 (br s, $1 \mathrm{H}, 1 \mathrm{c}-\mathrm{H}), 5.315 .43$ (m, $\left.5 \mathrm{H}, \mathrm{CH}-\mathrm{Ala}, 2^{\prime}-\mathrm{H}\right)$. ${ }^{13} \mathrm{C}$ NMR (150.9 MHz, D $\left.2 \mathrm{O}\right): \delta=14.2$ (2 C, Me), 15.9 (4 C, Ala-Me), 22.6 (1 C, NHAc), 25.4 (2 C, $\mathrm{COCH}_{2} \mathrm{CH}_{2} \mathrm{R}$ ), 30.5 (20 C, $\mathrm{CH}_{2}$-chain), 34.5 (2 C, $\mathrm{COCH}_{2} \mathrm{CH}_{2} \mathrm{R}$ ), 49.2 (4 C, $\mathrm{CHNH}_{3}{ }^{+}$), 54.0 (1 C, C-2c), 60.9 (1 C, C-6c), 62.4 (1 C, C-18), 64.0 (C CH CH-Glyc, $^{-}$ C-3'), 65.5 (C $\mathrm{CH}_{2-\text { GlycGlcNAc}}$ ), 66.8 (1 C, C-16), 68.2 (1 C, C-1'), 70.3 (1 C, C-4c), 71.1 (1 C, C-17), 71.4 (1 C, C-3c), 72.4 (1 C, C-5c), 73.4 (2 C, C-2a, C-2b), 74.4 (4 C, CH-Ala), 75.9 (2 C, C-3a, C-3b), 76.2 (1 C, C $\left.\mathrm{CH}_{\text {GlcNAc}}\right), \quad 97.2$ (1 C, C-1c). MALDI-MS (negative Mode, Matrix THAP, $\mathrm{CH}_{3} \mathrm{CN} / \mathrm{H}_{2} \mathrm{O}$ 3:2): $[\mathrm{M}-\mathrm{H}], \quad m / z=2247.9 ;$ found: $m / z=2248.5$; $\quad[(\mathrm{M}-$ Ala) $-\mathrm{H}], m / z=2176.9$; found: $m / z=2177.6$.

\subsection{Compound 23b}

Compound 23b was synthesized following the same method as for 23a, but using (Z)-L-alanine triethylammonium salt. Compound $22(250 \mathrm{mg}, 0.072 \mathrm{mmol})$ yielded 23b (190 mg, 62\%) as a colourless syrup. TLC (toluene/acetone 1:1): $R_{\mathrm{f}}=0.71, R_{\mathrm{f}}=0.75 .[\alpha]_{\mathrm{D}}+8.5(c$ $\left.0.18, \mathrm{CHCl}_{3}\right) .{ }^{1} \mathrm{H} \mathrm{NMR}\left(600 \mathrm{MHz} \mathrm{CDCl}_{3}\right): \delta=0.87(\mathrm{t}$, $6 \mathrm{H}, \mathrm{Me}), 1.101 .40\left(\mathrm{~m}, 52 \mathrm{H}, \mathrm{CH}_{2}\right.$-chain, Ala-Me), $1.461 .62\left(\mathrm{~m}, 4 \mathrm{H}, \mathrm{COCH}_{2} \mathrm{CH}_{2} \mathrm{R}\right), 1.821 .99(\mathrm{~m}, 3 \mathrm{H}$, NHAc), $2.092 .26\left(\mathrm{~m}, 4 \mathrm{H}, \mathrm{COCH}_{2} \mathrm{CH}_{2} \mathrm{R}\right), 3.32(\mathrm{~m}, 1$ $\mathrm{H}, 5 \mathrm{a} / \mathrm{b}-\mathrm{H}), 3.33(\mathrm{~m}, 1 \mathrm{H}, 2 \mathrm{a}-\mathrm{H}), 3,35$ (m, $\left.1 \mathrm{H}, 1^{\prime}-\mathrm{H}\right)$, 3.37 (m, $1 \mathrm{H}, 5 \mathrm{a} / \mathrm{b}-\mathrm{H}), 3.38(\mathrm{~m}, 1 \mathrm{H}, 2 \mathrm{~b}-\mathrm{H}), 3.43,3.45$ (m, $2 \mathrm{H}, 4 \mathrm{a}-\mathrm{H}, 4 \mathrm{~b}-\mathrm{H}), 3.50$ (m, $2 \mathrm{H}, 18-\mathrm{H}), 3.57$ (m, 2 H, 3a-H, 3b-H), 3.59 (m, 2 H, 6c-H), 3.63 (m, 1 H, 6a$\mathrm{H}), 3.67(\mathrm{~m}, 1 \mathrm{H}, 4 \mathrm{c}-\mathrm{H}), 3.68(\mathrm{~m}, 1 \mathrm{H}, 3 \mathrm{c}-\mathrm{H}), 3.71(\mathrm{~m}$, $2 \mathrm{H}, 2-\mathrm{H}, 17-\mathrm{H}), 3.76$ (m, $1 \mathrm{H}, 5 \mathrm{c}-\mathrm{H}), 3.83$ (m, $1 \mathrm{H}, 1^{\prime}-$ $\mathrm{H}), 4.01$ (m, $20 \mathrm{H}, 1-\mathrm{H}, 3-\mathrm{H}, 4-\mathrm{H}, 6-\mathrm{H}, 7-\mathrm{H}, 9-\mathrm{H}, 10-$ $\mathrm{H}, 12-\mathrm{H}, 13-\mathrm{H}, 15-\mathrm{H}), 4.07$ (m, $\left.2 \mathrm{H}, 3^{\prime}-\mathrm{H}, 16-\mathrm{H}\right), 4.09$ (m, $1 \mathrm{H}, 6 \mathrm{a}-\mathrm{H}), 4.14(\mathrm{~m}, 1 \mathrm{H}, 6 \mathrm{~b}-\mathrm{H}), 4.15\left(\mathrm{~m}, 1 \mathrm{H}, 3^{\prime}-\right.$ $\mathrm{H}), 4.17(\mathrm{~m}, 1 \mathrm{H}, 16-\mathrm{H}), 4.21(\mathrm{~m}, 2 \mathrm{H}, 6 \mathrm{~b}-\mathrm{H}, 1 \mathrm{a}-\mathrm{H})$, $4.33(\mathrm{~m}, 4 \mathrm{H}, \mathrm{CHNHCbz}), 4.334 .92\left(\mathrm{~m}, 22 \mathrm{H}, \mathrm{CH}_{2} \mathrm{Ph}\right)$, 4.34 (m, $1 \mathrm{H}, 2 \mathrm{c}-\mathrm{H}), 4.43$ (m, $1 \mathrm{H}, 1 \mathrm{~b}-\mathrm{H}), 4.79$ (m, $1 \mathrm{H}, 1 \mathrm{c}-$ $\mathrm{H}), 4.97\left(\mathrm{~m}, 4 \mathrm{H}, \mathrm{CH}_{2} \mathrm{Cbz}\right), 4.99\left(\mathrm{~m}, 12 \mathrm{H}, \mathrm{POCH}_{2} \mathrm{Ph}\right)$, $5.05\left(\mathrm{~m}, 4 \mathrm{H}, \mathrm{CH}_{2} \mathrm{Cbz}\right), 5.07\left(\mathrm{~m}, 1 \mathrm{H}, 2^{\prime}-\mathrm{H}\right), 5.09$ (m, 4 $\mathrm{H}, 5,8,11,14-\mathrm{H}), 5.596 .17(\mathrm{NH}), 7.047 .45(\mathrm{~m}, 105$ $\mathrm{H}, \mathrm{Ph}) .{ }^{13} \mathrm{C} \mathrm{NMR}\left(150.9 \mathrm{MHz}, \mathrm{CDCl}_{3}\right): \delta=14.4(2 \mathrm{C}$, Me), 18.3 (4 C, Ala-Me), 23.1 (1 C, NHAc), 25.2 (2 C, $\left.\mathrm{COCH}_{2} \mathrm{CH}_{2} \mathrm{R}\right), 22.9,29.8,32.1\left(20 \mathrm{C}, \mathrm{CH}_{2}\right.$-chain $)$, 34.2, $34.5\left(2 \mathrm{C}, \mathrm{COCH}_{2} \mathrm{R}\right), 50.1$ (4 C, CHNHCbz), 53.0 (1 C, C-2c), 63.4 (1 C, C-3'), 65.5 (10 C, C-1, C-3, C-4, C-6, C-7, C-9, C-10, C-12, C-13, C-15), 67.2 (4 C, $\mathrm{CH}_{2} \mathrm{Cbz}$ ), 67.3 (1 C, C-6b), 67.9 (1 C, C-16), 68.5 (1 C, C-1 ${ }^{\prime}$ ), 69.2 (2 C, C-6a, C-6c), 69.3 (1 C, C-18), 70.0 (1 $\left.\mathrm{C}, \mathrm{C}-2^{\prime}\right), 70.3$ (6 C, $\left.\mathrm{POCH} \mathrm{H}_{2} \mathrm{Ph}\right), 71.3$ (4 C, CH-Ala), 72.2 (1 C, C-5c), 72.676 .5 (11 C, $\left.\mathrm{CH}_{2} \mathrm{Ph}\right), 74.0$ (1 C, C-5a/b), 75.5 (1 C, C-4a/b), 77.0 (2 C, C-2, C-17), 77.8 (1 C, C-4a/b), 78.2 (1 C, C-4c), 78.5 (1 C, C-5a/b), 81.6 (1 C, C-3c), 82.7 (2 C, C-2a, C-2b), 85.2 (2 C, C-3a, C-3b), 100.2 (1 C, c-1c), 104.0 (1 C, C-1a), 104.4 (1 C, C-1b). MALDI-MS (positive Mode, Matrix p-nitroaniline+NaI, THF): $[\mathrm{M}+\mathrm{Na}]^{+}, \mathrm{m} / \mathrm{z}=4340$; found: $\mathrm{m} /$ $z=$ 4337.6. $\mathrm{C}_{232} \mathrm{H}_{281} \mathrm{~N}_{5} \mathrm{O}_{62} \mathrm{P}_{6}$ (4317.6): Calcd: C, 64.48; H, 6.51; N, 1.62. Found: C, 64.11; H, 6.68; N, 1.54 .

\subsection{Target molecule $1 b$}

Compound 1b was obtained using the same procedure as for 1a. Compound 23b (83 mg, $0.019 \mathrm{mmol})$ yielded $2(17 \mathrm{mg}, 40 \%)$ as white powder. $[\alpha]_{\mathrm{D}}+5.1$ (c 0.17 , $\mathrm{H}_{2} \mathrm{O}$ ). ${ }^{1} \mathrm{H}$ NMR (600 MHz, $\left.\mathrm{D}_{2} \mathrm{O}\right): \delta=0.800 .96$ (br s, $6 \mathrm{H}, \mathrm{Me}), 1.131 .46\left(\mathrm{~m}, 40 \mathrm{H}, \mathrm{CH}_{2}\right.$-chain), 1.531 .77 (m, $16 \mathrm{H}, \mathrm{Ala}-\mathrm{Me}, \mathrm{COCH}_{2} \mathrm{CH}_{2} \mathrm{R}$ ), 2.10 (s, $3 \mathrm{H}, \mathrm{NHAc}$ ), $2.272 .49\left(\mathrm{~m}, 4 \mathrm{H}, \mathrm{COCH}_{2} \mathrm{CH}_{2} \mathrm{R}\right), 3.254 .62(\mathrm{~m}, 54 \mathrm{H})$, 5.09 (br s, $1 \mathrm{H}, 1 \mathrm{c}-\mathrm{H}), 5.295 .46$ (m, $\left.5 \mathrm{H}, \mathrm{CH}-\mathrm{Ala}, 2^{\prime}-\mathrm{H}\right)$. ${ }^{13} \mathrm{C}$ NMR (150.9 MHz, D $\left.\mathrm{O}\right): \delta=16.7$ (2 C, Me), 18.2 (4 C, Ala-Me), 25.0 (1 C, NHAc), 27.6 (2 C, $\mathrm{COCH}_{2} \mathrm{CH}_{2} \mathrm{R}$ ), 25.5/33.1/34.9 (20 C, $\mathrm{CH}_{2}$-chain), 37.0 $\left(2 \mathrm{C}, \mathrm{COCH}_{2} \mathrm{CH}_{2} \mathrm{R}\right), 51.7\left(4 \mathrm{C}, \mathrm{CHNH}_{3}{ }^{+}\right), 56.5(1 \mathrm{C}$, C-2c), 63.7 (1 C, C-6c), 65.1 (1 C, C-18), 66.3 (1 C, C$\left.3^{\prime}\right), 66.6$ ( $\left.\mathrm{C} \mathrm{CH}_{2-G l y c}\right), 68.1$ ( $\left.\mathrm{C} \mathrm{CH}_{2-G l y c G l c N A c}\right), 69.4$ (1 C, C-16), 73.1 (1 C, C-4c), 73.7 (1 C, C-17), 74.0 (1 C, C-3c), 75.2 (1 C, C-5c), 76.2 (2 C, C-2a, C-2b), 77.0 (4 C, CH-Ala), 78.7 (2 C, C-3a, C-3b), 79.0 (1 C, $\mathrm{CH}_{\mathrm{Glc}-}$ NAc), 99.9 (1 C, C-1c). MALDI-MS (negative Mode, Matrix THAP, $\mathrm{CH}_{3} \mathrm{CN} / \mathrm{H}_{2} \mathrm{O}$ 3:2): $[\mathrm{M}-\mathrm{H}], \mathrm{m} /$ $z=2247.9$; found: $m / z=2245.5$; [(M-Ala) $-\mathrm{H}], \mathrm{m} /$ $z=2176.9$; found: $m / z=2174.8$. MALDI-MS (positive Mode, Matrix HCCA, $\left.\mathrm{H}_{2} \mathrm{O}\right)$ : $[\mathrm{M}+\mathrm{H}]^{+}, m / z=2249.9$; found: $m / z=2249.9$.

\subsection{1-O-(tert-Butyldiphenylsilyl)-2-O-(4-methoxyben- zyl)-3-O-monomethoxytrityl-sn-glycerol (25)}

Compound $24^{13}(3.56 \mathrm{~g}, 7.9 \mathrm{mmol})$ was dissolved in $\mathrm{CH}_{2} \mathrm{Cl}_{2}$ /pyridine $(1: 1,80 \mathrm{~mL})$, monomethoxytrityl chloride was added and the reaction mixture was stirred overnight at $\mathrm{rt}$. The solvent was removed in vacuo and the residue was dissolved in EtOAc, washed with saturated $\mathrm{NaHCO}_{3}$ solution, dried over $\mathrm{MgSO}_{4}$, and the solvent was removed in vacuo. Flash chromatography (petroleum ether/EtOAc 20:1) yielded 25 (5.6 g, 98\%) as a colourless crystals. TLC (petroleum ether/EtOAc 7:1): $R_{\mathrm{f}}=0.39$. Mp $118^{\circ} \mathrm{C} .-[\alpha]_{\mathrm{D}}+2.3$ (c $\left.1, \mathrm{CHCl}_{3}\right)$. ${ }^{1} \mathrm{H}$ NMR $\left(250 \mathrm{MHz}, \mathrm{CDCl}_{3}\right): \delta=0.96(\mathrm{~s}, 9 \mathrm{H}, t-\mathrm{Bu})$, 3.183 .33 (m, 2 H, $\left.\mathrm{CH}_{2 \mathrm{Glyc}}\right) 3.673 .91$ (m, 9 H, $\mathrm{CH}_{2 \mathrm{Glyc}}$, $\left.\mathrm{CH}_{\mathrm{Glyc}}, \mathrm{OMe}\right), 4.59$ (s, $\left.2 \mathrm{H}, \mathrm{CH}_{2} \mathrm{Ph}\right), 6.746 .91(\mathrm{~m}, 4 \mathrm{H}$,

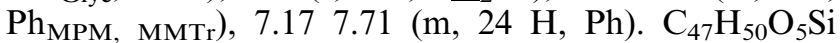
(723.0): Calcd: C, 78.08; H, 6.97. Found: C, 78.12; H, 7.02 .

\subsection{2-O-(4-Methoxybenzyl)-3-O-monomethoxytrityl-sn- glycerol (6)}

Compound 25 (5.5 g, $7.6 \mathrm{mmol})$ was dissolved in THF (100 mL) and TBAF (9.1 mL, $1 \mathrm{M}, 1.2$ equiv) was added dropwise. The reaction mixture was stirred for $2 \mathrm{~h}$ at $\mathrm{rt}$, and the solution was diluted with EtOAc, washed with water, saturated $\mathrm{NaHCO}_{3}$ solution and dried over $\mathrm{MgSO}_{4}$. The solvent was removed in vacuo. Flash chromatography (petroleum ether/EtOAc 3:1 $\rightarrow$ 2:1) yielded $6(3.5 \mathrm{~g}, 96 \%)$ as a colourless syrup. TLC (petroleum ether/EtOAc 6:1): $R_{\mathrm{f}}=0.07 .[\alpha]_{\mathrm{D}}+25\left(c 1, \mathrm{CHCl}_{3}\right) .{ }^{1} \mathrm{H}$ NMR (250 MHz, $\left.\mathrm{CDCl}_{3}\right): \delta=1.952 .05(\mathrm{~m}, 1 \mathrm{H}, \mathrm{OH})$, $3.173 .36\left(\mathrm{~m}, 2 \mathrm{H}, \mathrm{CH}_{2 \mathrm{Glyc}}\right) 3.593 .91$ (m, $6 \mathrm{H}, \mathrm{CH}_{2 \mathrm{Glyc}}$, $\left.\mathrm{CH}_{\mathrm{Glyc}}, \mathrm{OMe}\right), 4.47\left(\mathrm{~d}, 1 \mathrm{H}, J_{\text {gem }}=11.3 \mathrm{~Hz}, \mathrm{CH}_{2} \mathrm{Ph}\right)$, $4.62\left(\mathrm{~d}, 1 \mathrm{H}, J_{\text {gem }}=11.3 \mathrm{~Hz}, \mathrm{CH}_{2} \mathrm{Ph}\right), 6.796 .97(\mathrm{~m}, 4$ $\mathrm{H}, \mathrm{Ph}_{\text {MPM, ммтr), }} 7.187 .56(\mathrm{~m}, 14 \mathrm{H}, \mathrm{Ph}) . \mathrm{C}_{31} \mathrm{H}_{32} \mathrm{O}_{5}$ 
(484.6): Calcd: C, 76.66; H, 6.61. Found: C, 76.34; H, 6.86 .

\subsection{3-O-Allyl-2-O-benzyl-1-O-tert-butyldiphenylsilyl- sn-glycerol (27)}

To a solution of $26^{13}$ (15 g, $\left.40.5 \mathrm{mmol}\right)$ in DMF were added benzyl bromide $(7.24 \mathrm{~mL}, 60.7 \mathrm{mmol})$ and $\mathrm{NaH}$ ( $2 \mathrm{~g}, 2$ equiv) portionwise. The reaction mixture was stirred for $3 \mathrm{~h}$. The solvent was evaporated in vacuo and the residue was dissolved in EtOAc, washed with saturated $\mathrm{NH}_{4} \mathrm{Cl}$ solution and dried over $\mathrm{MgSO}_{4}$. The solvent was removed in vacuo. Flash chromatography (petroleum ether/EtOAc 30:1) yielded $27(13.4 \mathrm{~g}, 72 \%)$ as a colourless syrup. TLC (petroleum ether/EtOAc 7:1): $R_{\mathrm{f}}=0.49 . \quad[\alpha]_{\mathrm{D}}-1.3 \quad(c \quad 1$, acetone $) .{ }^{1} \mathrm{H} \quad \mathrm{NMR}$ $\left(250 \mathrm{MHz}, \mathrm{CDCl}_{3}\right): \delta=1.05(\mathrm{~s}, 9 \mathrm{H}, t-\mathrm{Bu}), 3.52$ 3.87, $3.964 .10\left(\mathrm{~m}, 7 \mathrm{H}, \mathrm{CH}_{2 \mathrm{Glyc}} \mathrm{H}, \mathrm{CH}_{\mathrm{Glyc}} \mathrm{H}, \mathrm{CH}_{2} \mathrm{CHCH}_{2}\right)$, $4.64\left(\mathrm{~s}, 2 \mathrm{H}, \mathrm{CH}_{2} \mathrm{Ph}\right), 5.115 .35\left(\mathrm{~m}, 2 \mathrm{H}, \mathrm{CH}_{2} \mathrm{CHCH}_{2}\right)$, $5.816 .01\left(\mathrm{~m}, 1 \mathrm{H}, \mathrm{CH}_{2} \mathrm{CHCH}_{2}\right), 7.217 .53,7.617 .81$ (m, $15 \mathrm{H}, \mathrm{Ph}) . \mathrm{C}_{29} \mathrm{H}_{36} \mathrm{O}_{3} \mathrm{Si}$ (460.7): Calcd: C, 75.61; H, 7.88. Found: C, 75.37; H, 7.90.

\subsection{2-O-Benzyl-1-O-tert-butyldiphenylsilyl-sn-glycerol} (28)

Compound 27 (13.3 g, $28.9 \mathrm{mmol})$ was dissolved in dry EtOH $(300 \mathrm{~mL}), \quad$ DBU $(648 \mu \mathrm{L}, 0.15$ equiv) and $\left(\mathrm{Ph}_{3} \mathrm{P}\right)_{3} \mathrm{RhCl}$ (8 g, 0.3 equiv) were added and the reaction mixture was stirred at $90^{\circ} \mathrm{C}$. After $15 \mathrm{~min}$, the propenyl intermediate was formed $\left(R_{\mathrm{f}}=0.76\right.$, toluene/ acetone 1.5:1); the solvent was removed in vacuo and the residue was dissolved in a solution of $1 \mathrm{M} \mathrm{HCl} /$ acetone $(1: 9,200 \mathrm{~mL})$ and stirred at $70{ }^{\circ} \mathrm{C}$ for $15 \mathrm{~min}$. The solution was neutralized with $\mathrm{NEt}_{3}$, diluted with EtOAc and washed with saturated $\mathrm{NaHCO}_{3}$ solution. After drying with $\mathrm{MgSO}_{4}$, the solvent was removed in vacuo. Flash chromatography (petroleum ether/EtOAc 3:1) yielded $28(9.2 \mathrm{~g}, 76 \%)$ as a yellow syrup. The analytical data are in accordance with those reported in the literature. ${ }^{21}$

\subsection{2-O-Benzyl-3-O-monomethoxytrityl-1-O-(tert-buty- Idiphenylsilyl)-sn-glycerol (29)}

Compound 29 was obtained as described for $\mathbf{2 5}$. Compound 28 (9.15 g, $21.75 \mathrm{mmol})$, monomethoxytrityl chloride $(8.2 \mathrm{~g}, 26.55 \mathrm{mmol})$. Flash chromatography (PE/EE 20:1) yielded $29(14.77 \mathrm{~g}, 98 \%)$ as a colourless syrup. TLC (petroleum ether/EtOAc 7:1): $R_{\mathrm{f}}=0.48$. $[\alpha]_{\mathrm{D}}+1.9\left(c 1, \mathrm{CHCl}_{3}\right) .{ }^{1} \mathrm{H}$ NMR $\left(250 \mathrm{MHz}, \mathrm{CDCl}_{3}\right)$ : $\delta=0.96(\mathrm{~s}, 9 \mathrm{H}, t-\mathrm{Bu}), 3.213 .35\left(\mathrm{~m}, 2 \mathrm{H}, \mathrm{CH}_{2 \mathrm{Glyc}} \mathrm{H}\right)$ $3.693 .85\left(\mathrm{~m}, 6 \mathrm{H}, \mathrm{CH}_{2 \text {-Glyc }} \mathrm{H}, \mathrm{CH}_{\mathrm{Glyc}} \mathrm{H}, \mathrm{OMe}\right), 4.67$ (s, $\left.2 \mathrm{H}, \mathrm{CH}_{2} \mathrm{Ph}_{\mathrm{MPM}}\right), 6.746 .83\left(\mathrm{~m}, 2 \mathrm{H}, \mathrm{Ph}_{\mathrm{MPM}}\right)$, $7.157 .51, \quad 7.587 .69(\mathrm{~m}, 27 \mathrm{H}, \quad \mathrm{Ph}) . \mathrm{C}_{46} \mathrm{H}_{48} \mathrm{O}_{4} \mathrm{Si}$ (693.0): Calcd: C, 79.73; H, 6.98. Found: C, 79.80; H, 7.09.

\subsection{MPM2-O-Benzyl-3-O-monomethoxytrityl-sn-glyc- erol (30)}

Compound 29 (16 g, $23.1 \mathrm{mmol}$ ) was dissolved in THF $(300 \mathrm{~mL})$ and TBAF-solution $(27 \mathrm{~mL}, 1 \mathrm{M}$ in THF,
1.2 equiv) was added dropwise. The reaction mixture was stirred for $3 \mathrm{~h}$, diluted with EtOAc and washed with saturated $\mathrm{NaHCO}_{3}$ solution, the organic phase was dried over $\mathrm{MgSO}_{4}$ and the solvent was removed in vacuo. Flash chromatography (petroleum ether/EtOAc 3:1) yielded $30(9.86 \mathrm{~g}, 98 \%)$ as a colourless syrup. TLC (petroleum ether/EtOAc 7:1): $R_{\mathrm{f}}=0.13$. $[\alpha]_{\mathrm{D}}+23.6(c$ 1, $\left.\mathrm{CHCl}_{3}\right) .{ }^{1} \mathrm{H}$ NMR $\left(250 \mathrm{MHz}, \mathrm{CDCl}_{3}\right): \delta=1.982 .05$ $(\mathrm{m}, 1 \mathrm{H}, \mathrm{OH}), 3.23\left(\mathrm{dd}, 1 \mathrm{H}, J_{\text {gem }}=9.9 \mathrm{~Hz}\right.$, $\left.J_{\text {vic }}=4.9 \mathrm{~Hz}, \mathrm{CH}_{2 \text {-Glyc }} \mathrm{H}\right) 3.30\left(\mathrm{dd}, 1 \mathrm{H}, J_{\text {gem }}=9.9 \mathrm{~Hz}\right.$, $\left.J_{\text {vic }}=4.7 \mathrm{~Hz}, \mathrm{CH}_{2 \mathrm{Glyc}} \mathrm{H}\right), 3.623 .79\left(\mathrm{~m}, 3 \mathrm{H}, \mathrm{CH}_{2-\mathrm{Glyc}}\right.$, $\left.\mathrm{CH}_{\mathrm{Glyc}}\right), 3.79(\mathrm{~s}, 3 \mathrm{H}, \mathrm{OMe}), 4.54\left(\mathrm{~d}, 1 \mathrm{H}, J_{\text {gem }}=11.7\right.$ $\left.\mathrm{Hz}, \mathrm{CH}_{2} \mathrm{Ph}\right), 4.69\left(\mathrm{~d}, 1 \mathrm{H}, J_{\text {gem }}=11.7 \mathrm{~Hz}, \mathrm{CH}_{2} \mathrm{Ph}\right)$, $6.806 .88\left(\mathrm{~m}, 2 \mathrm{H}, \mathrm{Ph}_{\mathrm{MPM}}\right), 7.197 .52(\mathrm{~m}, 17 \mathrm{H}, \mathrm{Ph})$. $\mathrm{C}_{30} \mathrm{H}_{30} \mathrm{O}_{4} 0.25 \mathrm{H}_{2} \mathrm{O}$ (459.1): Calcd: C, 78.49; H, 6.70. Found: C, 78.45; H, 6.82.

\subsection{7. [Benzyloxy]-[diisopropylamino]-[2- $O$-benzyl-3- $O$ - monomethoxytrityl-sn-glycero|phosphane (7)}

Compound 7 was obtained as described for 3 . Tetrazole (108 mg, 0.6 equiv), 30 ( $2.03 \mathrm{~g}, 7.46 \mathrm{mmol})$, benzyloxybis-(diisopropyl amine)-phosphane (1.37 g, 1.2 equiv). Flash chromatography (petroleum ether/EtOAc 8:1, $\left.+1 \% \mathrm{NEt}_{3}\right)$ yielded 7 (2.4 g, quant.) as a colourless oil. TLC (petroleum ether/EtOAc 7:1, $1 \% \mathrm{NEt}_{3}$ ): $R_{\mathrm{f}}=0.54 . \quad[\alpha]_{\mathrm{D}}-0.5 \quad\left(c \quad 1, \quad \mathrm{CHCl}_{3}\right) .{ }^{1} \mathrm{H} \quad \mathrm{NMR}$ $\left(250 \mathrm{MHz}, \mathrm{CDCl}_{3}\right): \delta=1.101 .30\left(\mathrm{~m}, 12 \mathrm{H}, \mathrm{CH}\left(\mathrm{CH}_{3}\right)_{2}\right)$, 3.193 .34 (m, $\left.2 \mathrm{H}, \mathrm{CH}_{2-\mathrm{Glyc}}\right), 3.473 .91$ (m, 8 H, OMe,

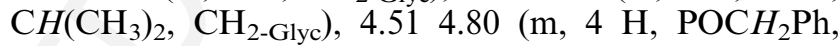
$\left.\mathrm{CH}_{2} \mathrm{Ph}\right), 6.736 .89\left(\mathrm{~m}, 2 \mathrm{H}, \mathrm{Ph}_{M M T r}\right), 7.137 .58(\mathrm{~m}, 22$ $\mathrm{H}, \mathrm{Ph}) . \mathrm{C}_{43} \mathrm{H}_{50} \mathrm{NO}_{5} \mathrm{P}(691.8)$ : Calcd: C, 74.65; H, 7.28; N, 2.02. Found: C, 74.64; H, 7.35; N, 2.02 .

\subsection{Compound 31}

Compound 31 was synthesized following the general coupling procedure. Compound $6(3.59 \mathrm{~g}, 7.41 \mathrm{mmol})$, tetrazole $(780 \mathrm{mg}, 11.14 \mathrm{mmol})$ and phosphite amide $5^{13}$ (5.6 g, 1.1 equiv) were dissolved in dry $\mathrm{CH}_{2} \mathrm{Cl}_{2}$ $(150 \mathrm{~mL})$. (TLC, petroleum ether/EtOAc: 7:1, $+1 \%$ $\left.\mathrm{NEt}_{3} ; R_{\mathrm{f}}=0.22\right)$. Oxidation with $t-\mathrm{BuO}_{2} \mathrm{H}(4 \mathrm{~mL})$ and flash chromatography (petroleum ether/EtOAc 3:1) yielded $31(7.89 \mathrm{~g}, 98 \%)$ as a colourless syrup. TLC (petroleum ether/EtOAc 1:1): $R_{\mathrm{f}}=0.69 .[\alpha]_{\mathrm{D}}-0.4(c 1$, $\left.\mathrm{CHCl}_{3}\right) \cdot{ }^{1} \mathrm{H}$ NMR $\left(250 \mathrm{MHz}, \mathrm{CDCl}_{3}\right): \delta=1.01$ (s, 9 $\mathrm{H}, t-\mathrm{Bu}), 3.13-3.22(\mathrm{~m}, 2 \mathrm{H}, 6-\mathrm{H}), 3.583 .88(\mathrm{~m}, 13 \mathrm{H}$, 1, 2-H, 5-H, OMe), $4.034 .31(\mathrm{~m}, 4 \mathrm{H}, 3-\mathrm{H}, 4-\mathrm{H})$, $4.414 .57\left(\mathrm{~m}, 4 \mathrm{H}, \mathrm{CH}_{2}-\mathrm{Ph}\right), 4.925 .01(\mathrm{~m}, 2 \mathrm{H}$, $\left.\mathrm{POCH}_{2}-\mathrm{Ph}\right), 6.726 .88\left(\mathrm{~m}, 6 \mathrm{H}, \mathrm{Ph}_{\text {MPM, MMTr }}\right), 7.10$ 7.50, $7.607 .70(\mathrm{~m}, 31 \mathrm{H}, \mathrm{Ph})$. MALDI-MS (positive Mode, Matrix DHB, THF): $[\mathrm{M}+\mathrm{Na}]^{+}, \mathrm{m} / z=1110.3$; found: $m / z=1110.4$. $\mathrm{C}_{65} \mathrm{H}_{71} \mathrm{O}_{11} \mathrm{PSi} * 1 / 2 \mathrm{H}_{2} \mathrm{O}(1096.3)$ : Calcd: C, 71.21; H, 6.62. Found: C, 71.10; H, 6.68.

5.29. 3-O-[2-O-(4-Methoxybenzyl)-3-O-monomethoxytrityl-sn-glycero-(1)-benzylphosphol-2-O-(4-methoxybenzyl)-sn-glycerol (32)

Comoound 32 was synthesized following the general deprotection procedure. 31 (7.78 g, $7.16 \mathrm{mmol})$, TBAFsolution $(8.60 \mathrm{~mL}, 1.2$ equiv), THF $(150 \mathrm{~mL})$ and flash chromatography (petroleum ether/EtOAc $1: 1 \rightarrow 1: 3$ ) 
yielded $32(5 \mathrm{~g}, 82 \%)$ as a colourless syrup. TLC (toluene/acetone 1:1): $R_{\mathrm{f}}=0.61$. $[\alpha]_{\mathrm{D}}+12.5\left(c 1, \mathrm{CHCl}_{3}\right)$. ${ }^{1} \mathrm{H}$ NMR $\left(250 \mathrm{MHz}, \mathrm{CDCl}_{3}\right): \delta=3.163 .25(\mathrm{~m}, 2 \mathrm{H}$, 6-H), 3.483 .89 (m, 13 H, 1-H, 2-H, 5-H, OMe), 4.01 $4.30(\mathrm{~m}, 4 \mathrm{H}, 3-\mathrm{H}, 4-\mathrm{H}), 4.394 .60\left(\mathrm{~m}, 4 \mathrm{H}, \mathrm{CH}_{2}-\mathrm{Ph}\right)$, $4.965 .05\left(\mathrm{~m}, 2 \mathrm{H}, \mathrm{POCH}_{2}-\mathrm{Ph}\right), 6.766 .91(\mathrm{~m}, 6 \mathrm{H}$, $\mathrm{Ph}_{\text {MPM, мMTr }}$ ), 7.157 .49 (m, 21 H, Ph). MALDI-MS (positive Mode, Matrix DHB, THF): $[\mathrm{M}+\mathrm{Na}]^{+}, \mathrm{m} /$ $z=871.9 ;$ found: $m / z=872.2 . \quad \mathrm{C}_{49} \mathrm{H}_{53} \mathrm{O}_{11} \mathrm{P} \quad 0.5 \mathrm{H}_{2} \mathrm{O}$ (857.9): Calcd: C, 68.60; H, 6.35. Found: C, 68.61; H, 6.40 .

\subsection{Diphosphate 33}

Compound 33 was synthesized following the general coupling procedure. $32(5.13 \mathrm{~g}, 6.04 \mathrm{mmol})$, tetrazole $(635 \mathrm{mg}, 9.07 \mathrm{mmol})$ and phosphite amide $\mathbf{5}^{13}(5.0 \mathrm{~g}$, 1.2 equiv) were dissolved in dry $\mathrm{CH}_{2} \mathrm{Cl}_{2}(120 \mathrm{~mL})$. (TLC petroleum ether/EtOAc: $1: 1, \quad+1 \% \quad \mathrm{NEt}_{3}$, $\left.R_{\mathrm{f}}=0.68\right)$. Oxidation with $t-\mathrm{BuO}_{2} \mathrm{H}(3.5 \mathrm{~mL})$. Flash chromatography (petroleum ether/EtOAc 2:1 $\rightarrow$ 1:1) yielded $33(8.4 \mathrm{~g}, 96 \%)$ as a colourless syrup. TLC (petroleum ether/EtOAc 1:1): $R_{\mathrm{f}}=0.35$. $[\alpha]_{\mathrm{D}}-1.1(c 1$, $\mathrm{CHCl}_{3}$ ). ${ }^{1} \mathrm{H}$ NMR $\left(250 \mathrm{MHz}, \mathrm{CDCl}_{3}\right): \delta=1.02$ (s, 9 $\mathrm{H}, t-\mathrm{Bu}), 3.143 .22(\mathrm{~m}, 2 \mathrm{H}, 9-\mathrm{H}), 3.593 .82(\mathrm{~m}, 17 \mathrm{H}$, 1-H, 2-H, 5-H, 8-H, OMe), 3.904 .32 (m, 8 H, 3-H, 4$\mathrm{H}, 6-\mathrm{H}, 7-\mathrm{H}), 4.394 .54\left(\mathrm{~m}, 6 \mathrm{H}, \mathrm{CH}_{2}-\mathrm{Ph}\right), 4.905 .02$ (m, $\left.4 \mathrm{H}, \mathrm{POCH} \mathrm{H}_{2}-\mathrm{Ph}\right), 6.696 .88\left(\mathrm{~m}, 8 \mathrm{H}, \mathrm{Ph}_{\mathrm{MPM}}\right.$ мMTr), 7.09 7.48, $7.597 .70(\mathrm{~m}, 38 \mathrm{H}, \mathrm{Ph})$. MALDI-MS (positive Mode, Matrix DHB, THF): $[\mathrm{M}+\mathrm{Na}]_{+}, \mathrm{ml}$ $z=1474.6 ; \quad$ found: $\quad m / z=1475.9 . \quad \mathrm{C}_{83} \mathrm{H}_{92} \mathrm{O}_{17} \mathrm{P}_{2} \mathrm{Si}$ (1451.6): Calcd: C, 68.67; H, 6.39. Found: C, 68.34; H, 6.37 .

\subsection{Diphosphate 34}

Compound 34 was synthesized following the general deprotection procedure. 33 ( $8.3 \mathrm{~g}, 5.72 \mathrm{mmol})$ TBAF-solution $(6.9 \mathrm{~mL}, 1.2$ equiv), THF $(150 \mathrm{~mL})$ and flash chromatography (toluene/acetone 2.5:1) yielded $\mathbf{3 4}$ $(6.34 \mathrm{~g}, 91 \%)$ as a colourless syrup. TLC (toluene/acetone 1:1): $R_{\mathrm{f}}=0.32 .[\alpha]_{\mathrm{D}}+5.1\left(c 1, \mathrm{CHCl}_{3}\right) .{ }^{1} \mathrm{H} \mathrm{NMR}$ $\left(250 \mathrm{MHz}, \mathrm{CDCl}_{3}\right): \delta=2.622 .71$ (br s, $\left.1 \mathrm{H}, \mathrm{OH}\right)$, $3.143 .23(\mathrm{~m}, 2 \mathrm{H}, 9-\mathrm{H}), 3.503 .87(\mathrm{~m}, 17 \mathrm{H}, 1-\mathrm{H}, 2-$ $\mathrm{H}, 5-\mathrm{H}, 8-\mathrm{H}, \mathrm{OMe}), 3.904 .28$ (m, $8 \mathrm{H}, 3-\mathrm{H}, 4-\mathrm{H}, 6-\mathrm{H}$, 7-H), $4.404 .59\left(\mathrm{~m}, 6 \mathrm{H}, \mathrm{CH}_{2}-\mathrm{Ph}\right), 4.915 .07(\mathrm{~m}, 4 \mathrm{H}$, $\left.\mathrm{POCH}_{2}-\mathrm{Ph}\right), 6.736 .89\left(\mathrm{~m}, 8 \mathrm{H}, \mathrm{Ph}_{\mathrm{MPM}}, \mathrm{MMTr}\right), 7.11$ 7.47 (m, $28 \mathrm{H}, \mathrm{Ph}$ ). MALDI-MS (positive Mode, Matrix DHB, THF): $[\mathrm{M}+\mathrm{Na}]^{+}, \quad m / z=1236.2 ;$ found: $m /$ $z=1236.0, \quad[\mathrm{M}+\mathrm{K}]^{+}, \quad m / z=1252.3 ; \quad$ found: $\quad \mathrm{m} /$ $z=1252.3 . \mathrm{C}_{67} \mathrm{H}_{74} \mathrm{O}_{17} \mathrm{P}_{2}$ (1213.2): Calcd: C, 66.33; H, 6.15. Found: $\mathrm{C}, 66.54 ; \mathrm{H}, 6.37$.

\subsection{Triphosphate 35}

Compound 35 was synthesized following the general coupling procedure. Compound $34(6.30 \mathrm{~g}, 5.19 \mathrm{mmol})$, tetrazole $(563 \mathrm{mg}, 8.04 \mathrm{mmol})$ and phosphite amide $\mathbf{5}^{13}$ (4.42 g, 1.2 equiv) were dissolved in dry $\mathrm{CH}_{2} \mathrm{Cl}_{2}$ $(120 \mathrm{~mL})$. (TLC toluene/acetone 3:1, +1\% $\mathrm{NEt}_{3}$, $\left.R_{\mathrm{f}}=0.62\right)$. Oxidation with $t-\mathrm{BuO}_{2} \mathrm{H}(3.3 \mathrm{~mL})$. Flash chromatography (toluene/acetone 4.5:1) yielded $\mathbf{3 5}$ $(8.82 \mathrm{~g}, 94 \%)$ as a colourless syrup. TLC (toluene/ace- tone 3:1): $R_{\mathrm{f}}=0.48 .[\alpha]_{\mathrm{D}}-0.7\left(c 1, \mathrm{CHCl}_{3}\right) .{ }^{1} \mathrm{H}$ NMR $\left(250 \mathrm{MHz}, \mathrm{CDCl}_{3}\right): \delta=1.02(\mathrm{~s}, 9 \mathrm{H}, t$-Bu), 3.133 .22 (m, $2 \mathrm{H}, 12-\mathrm{H}), 3.563 .88(\mathrm{~m}, 21 \mathrm{H}, 1-\mathrm{H}, 2-\mathrm{H}, 5-\mathrm{H}, 8-$ $\mathrm{H}, 11-\mathrm{H}, \mathrm{OMe}), 3.904 .32(\mathrm{~m}, 12 \mathrm{H}, 3-\mathrm{H}, 4-\mathrm{H}, 6-\mathrm{H}, 7-$ $\mathrm{H}, 9-\mathrm{H}, 10-\mathrm{H}), 4.394 .58\left(\mathrm{~m}, 8 \mathrm{H}, \mathrm{CH}_{2}-\mathrm{Ph}\right), 4.895 .08$ $\left(\mathrm{m}, 6 \mathrm{H}, \mathrm{POCH} \mathrm{H}_{2}-\mathrm{Ph}\right), 6.696 .89\left(\mathrm{~m}, 10 \mathrm{H}, \mathrm{Ph}_{\mathrm{MPM}}\right.$, MMTr $), 7.107 .50,7.597 .71(\mathrm{~m}, 45 \mathrm{H}, \mathrm{Ph})$. MALDIMS (positive Mode, Matrix DHB, THF): $[\mathrm{M}+\mathrm{Na}]^{+}$, $m / z=1838.97 ;$ found: $m / z=1838.8, \quad[\mathrm{M}+\mathrm{K}]^{+}, \quad m /$ $z=1855.1 ; \quad$ found: $\quad m / z=1856.3 . \quad \mathrm{C}_{101} \mathrm{H}_{113} \mathrm{O}_{23} \mathrm{P}_{3} \mathrm{Si}$ (1816.0): Calcd: C, 66.80; H, 6.27. Found: C, 67.30; H, 6.15 .

\subsection{Triphosphate 36}

To a solution of triphosphate $35(8.66 \mathrm{~g}, 4.77 \mathrm{mmol})$ in $\mathrm{CH}_{2} \mathrm{Cl}_{2}(50 \mathrm{~mL})$ and $\mathrm{MeOH}(100 \mathrm{~mL}), \mathrm{CSA}$ was added (222 mg, $0.95 \mathrm{mmol}, 0.2$ equiv); the reaction mixture was stirred for $2 \mathrm{~h}$ at rt. After neutralization with $\mathrm{NEt}_{3}$, the solvent was removed in vacuo and coevaporated with toluene. Flash chromatography (toluene/acetone 2.5:1) yielded $36(6.8 \mathrm{~g}, 92 \%)$ as a colourless syrup. TLC (toluene/acetone 2.5:1): $R_{\mathrm{f}}=0.30 .[\alpha]_{\mathrm{D}}-7.5\left(c 1, \mathrm{CHCl}_{3}\right)$. ${ }^{1} \mathrm{H}$ NMR $\left(250 \mathrm{MHz}, \mathrm{CDCl}_{3}\right): \delta=1.02(\mathrm{~s}, 9 \mathrm{H}, t$-Bu), 2.733 .0 (br s, $1 \mathrm{H}, \mathrm{OH}), 3.503 .86(\mathrm{~m}, 20 \mathrm{H}, 1-\mathrm{H}, 2-$ $\mathrm{H}, 5-\mathrm{H}, 8-\mathrm{H}, 11-\mathrm{H}, 12-\mathrm{H}, \mathrm{OMe}), 3.914 .34(\mathrm{~m}, 12 \mathrm{H}$, 3-H, 4-H, 6-H, 7-H, 9-H, 10-H), $4.394 .60(\mathrm{~m}, 8 \mathrm{H}$, $\left.\mathrm{CH}_{2}-\mathrm{Ph}\right), 4.935 .10\left(\mathrm{~m}, 6 \mathrm{H}, \mathrm{POCH}_{2}-\mathrm{Ph}\right), 6.716 .90$ $\left(\mathrm{m}, 8 \mathrm{H}, \mathrm{Ph}_{\text {MPM, MMTr}}\right), 7.107 .47,7.597 .70$ (m, 33 $\mathrm{H}, \mathrm{Ph}$ ). MALDI-MS (positive Mode, Matrix DHB, THF): $[\mathrm{M}+\mathrm{Na}]^{+}, m / z=1566.6$; found: $m / z=1567.3$, $[\mathrm{M}+\mathrm{K}]^{+}, \quad m / z=1582.7 ; \quad$ found: $\quad m / z=1582.4$. $\mathrm{C}_{81} \mathrm{H}_{97} \mathrm{O}_{22} \mathrm{P}_{3} \mathrm{Si}$ (1543.6): Calcd: C, 63.02; H, 6.33. Found: C, 63.02; H, 6.58.

\subsection{Tetraphosphate 37}

Compound 37 was synthesized following the general coupling procedure. Compound $36(2.5 \mathrm{~g}, 1.62 \mathrm{mmol})$, tetrazole $(170 \mathrm{mg}, 2.43 \mathrm{mmol})$ and phosphite amide 7 $\left(1.35 \mathrm{~g}, 1.2\right.$ equiv) were dissolved in dry $\mathrm{CH}_{2} \mathrm{Cl}_{2}$ $(60 \mathrm{~mL})$. (TLC toluene/acetone $3: 1,+1 \% \quad \mathrm{NEt}_{3}$, $\left.R_{\mathrm{f}}=0.62\right)$. Oxidation with $t-\mathrm{BuO}_{2} \mathrm{H}(2 \mathrm{~mL})$. Flash chromatography (toluene/acetone 2:1) yielded $37(3.34 \mathrm{~g}$, $96 \%$ ) as a colourless syrup. TLC (toluene/acetone $2: 1$ ): $R_{\mathrm{f}}=0.42 . \quad[\alpha]_{\mathrm{D}} \quad-1.1 \quad\left(c \quad 1, \quad \mathrm{CHCl}_{3}\right) .{ }^{1} \mathrm{H} \quad \mathrm{NMR}$ $\left(250 \mathrm{MHz}, \mathrm{CDCl}_{3}\right): \delta=1.02(\mathrm{~s}, 9 \mathrm{H}, t-\mathrm{Bu}), 3.173 .25$ (m, $2 \mathrm{H}, 15-\mathrm{H}), 3.593 .81(\mathrm{~m}, 19 \mathrm{H}, 1-\mathrm{H}, 2-\mathrm{H}, 5-\mathrm{H}, 8-$ $\mathrm{H}, 11-\mathrm{H}, 14-\mathrm{H}, \mathrm{OMe}), 3.894 .32(\mathrm{~m}, 16 \mathrm{H}, 3-\mathrm{H}, 4-\mathrm{H}$, 6-H, 7-H, 9-H, 10-H, 12-H, 13-H), 4.384 .51 (m, $8 \mathrm{H}$, $\left.\mathrm{CH}_{2}-\mathrm{Ph}\right), 4.514 .61\left(\mathrm{~m}, 2 \mathrm{H}, \mathrm{CH}_{2}-\mathrm{Ph}\right), 4.905 .04(\mathrm{~m}, 8$ $\left.\mathrm{H}, \mathrm{POCH} \mathrm{H}_{2} \mathrm{Ph}\right), 6.696 .88\left(\mathrm{~m}, 10 \mathrm{H}, \mathrm{Ph}_{\mathrm{MPM}}, \mathrm{MMTr}\right)$, $7.097 .49,7.587 .69(\mathrm{~m}, 55 \mathrm{H}, \mathrm{Ph}) . \mathrm{C}_{118} \mathrm{H}_{132} \mathrm{O}_{28} \mathrm{P}_{4} \mathrm{Si}$ (2150.3): Calcd: C, 65.91; H, 6.19. Found: C, 66.09; H, 6.16.

\subsection{Tetraphosphate 38}

Compound 38 was synthesized following the general deprotection procedure. 37 (3.3 g, $1.54 \mathrm{mmol})$, TBAF-solution ( $1.85 \mathrm{~mL}, 1.2$ equiv), THF $(60 \mathrm{~mL})$. Flash chromatography (toluene/acetone $2: 1 \rightarrow 1: 1)$ yielded $38(2.78 \mathrm{~g}$, $95 \%$ ) as a colourless syrup. TLC (toluene/acetone 1:1): 
$R_{\mathrm{f}}=0.53 .[\alpha]_{\mathrm{D}}+2.5\left(c 1, \mathrm{CHCl}_{3}\right) .{ }^{1} \mathrm{H}$ NMR $(250 \mathrm{MHz}$, $\left.\mathrm{CDCl}_{3}\right): \delta=2.752 .99($ br s, $1 \mathrm{H}, \mathrm{OH}), 3.143 .26(\mathrm{~m}, 2$ $\mathrm{H}, 15-\mathrm{H}), 3.503 .84$ (m, $19 \mathrm{H}, 1-\mathrm{H}, 2-\mathrm{H}, 5-\mathrm{H}, 8-\mathrm{H}, 11-$ $\mathrm{H}, 14-\mathrm{H}, \mathrm{OMe}), 3.894 .29$ (m, $16 \mathrm{H}, 3-\mathrm{H}, 4-\mathrm{H}, 6-\mathrm{H}, 7-$ $\mathrm{H}, 9-\mathrm{H}, 10-\mathrm{H}, 12-\mathrm{H}, 13-\mathrm{H}), 4.494 .63\left(\mathrm{~m}, 10 \mathrm{H}, \mathrm{CH}_{2}-\right.$ $\mathrm{Ph}), 4.895 .10\left(\mathrm{~m}, 8 \mathrm{H}, \mathrm{POCH} \mathrm{H}_{2} \mathrm{Ph}\right), 6.706 .92(\mathrm{~m}, 10$ $\mathrm{H}, \mathrm{Ph}_{\text {MPM, MMTr }}$ ), 7.107 .49 (m, $\left.45 \mathrm{H}, \mathrm{Ph}\right) . \mathrm{C}_{102} \mathrm{H}_{114} \mathrm{O}_{28} \mathrm{P}_{4}$ toluene (2004.0): Calcd: C, 65.33; H, 6.14. Found: C, 65.41; H, 6.12.

\subsection{Pentaphosphate 39}

Compound 39 was synthesized following the general coupling procedure. Compound $\mathbf{3 8}(2.71 \mathrm{~g}, 1.42 \mathrm{mmol})$, tetrazole $(150 \mathrm{mg}, 2.14 \mathrm{mmol})$ and phosphite amide $4^{13}$ (1.78 g, 1.2 equiv) were dissolved in dry $\mathrm{CH}_{2} \mathrm{Cl}_{2}$ (60 mL). (TLC toluene/acetone 3:1, +1\% $\mathrm{NEt}_{3}$, $\left.R_{\mathrm{f}}=0.62\right)$. Oxidation with $t-\mathrm{BuO}_{2} \mathrm{H}(2 \mathrm{~mL})$. Flash chromatography (toluene/acetone $2: 1 \rightarrow 1: 3$ ) yielded $\mathbf{3 9}$ $(3.60 \mathrm{~g}, 96 \%)$ as a colourless syrup. TLC (toluene/acetone 1:1): $R_{\mathrm{f}}=0.52, R_{\mathrm{f}}=0.60 .[\alpha]_{\mathrm{D}}+12.0\left(c 1, \mathrm{CHCl}_{3}\right)$. ${ }^{1} \mathrm{H}$ NMR $\left(250 \mathrm{MHz}, \mathrm{CDCl}_{3}\right): \delta=1.02(\mathrm{~s}, 9 \mathrm{H}, t$-Bu), 1.902 .03 (m, $3 \mathrm{H}, \mathrm{NHAc}), 3.103 .26$ (m, $3 \mathrm{H}, 18,6 \mathrm{c}-$ $\mathrm{H}), 3.413 .83(\mathrm{~m}, 27 \mathrm{H}, 3 \mathrm{c}-\mathrm{H}, 4 \mathrm{c}-\mathrm{H}, 5 \mathrm{c}-\mathrm{H}, 6 \mathrm{c}-\mathrm{H}, 1-\mathrm{H}$, 2-H, 5-H, 8-H, 11-H, 14-H, 17-H, OMe), 3.884 .80 (m, $38 \mathrm{H}, 1 \mathrm{c}-\mathrm{H}, 2 \mathrm{c}-\mathrm{H}, 3-\mathrm{H}, 4-\mathrm{H}, 6-\mathrm{H}, 7-\mathrm{H}, 9-\mathrm{H}, 10-\mathrm{H}$, $\left.12-\mathrm{H}, 13-\mathrm{H}, 15-\mathrm{H}, 16-\mathrm{H}, \mathrm{CH}_{2}-\mathrm{Ph}\right), 4.855 .05(\mathrm{~m}, 10 \mathrm{H}$, POC $\left.H_{2}-\mathrm{Ph}\right), 6.686 .88\left(\mathrm{~m}, 10 \mathrm{H}, \mathrm{Ph}_{\text {MPM, MMTr }}\right), 7.02$ $7.71(\mathrm{~m}, 75 \mathrm{H}, \mathrm{Ph}) . \mathrm{C}_{157} \mathrm{H}_{176} \mathrm{NO}_{38} \mathrm{P}_{5} \mathrm{Si}$ (2868.0): Calcd: C, 65.75; H, 6.19; N, 0.49. Found: C. 66.11; H, 6.44; $\mathrm{N}, 0.48$.

\subsection{Pentaphosphate 40}

To a solution of $39(3.53 \mathrm{~g}, 1.23 \mathrm{mmol})$ in $\mathrm{CH}_{2} \mathrm{Cl}_{2}$ $(60 \mathrm{~mL})$ and $\mathrm{MeOH}(20 \mathrm{~mL}), \mathrm{CSA}$ was added $(86 \mathrm{mg}$, $0.37 \mathrm{mmol}, 0.3$ equiv); the reaction mixture was stirred for $2 \mathrm{~h}$ at $\mathrm{rt}$. After neutralization with $\mathrm{NEt}_{3}$, the solvent was removed in vacuo and coevaporated with toluene. Flash chromatography (toluene/acetone $1: 1 \rightarrow 1: 3$ ) yielded $40(2.93 \mathrm{~g}, 92 \%)$ as a colourless syrup. TLC (toluene/acetone 1:1): $R_{\mathrm{f}}=0.55 .[\alpha]_{\mathrm{D}}+7.6\left(c 1, \mathrm{CHCl}_{3}\right) .{ }^{1} \mathrm{H}$ NMR (250 MHz, $\left.\mathrm{CDCl}_{3}\right): \delta=1.02(\mathrm{~s}, 9 \mathrm{H}, t-\mathrm{Bu}), 1.89$ $2.02(\mathrm{~m}, 3 \mathrm{H}, \mathrm{NHAc}), 3.113 .23(\mathrm{~m}, 1 \mathrm{H}, 6 \mathrm{c}-\mathrm{H}), 3.40$ $3.85(\mathrm{~m}, 26 \mathrm{H}, 3 \mathrm{c}-\mathrm{H}, 4 \mathrm{c}-\mathrm{H}, 5 \mathrm{c}-\mathrm{H}, 6 \mathrm{c}-\mathrm{H}, 1-\mathrm{H}, 2-\mathrm{H}, 5-\mathrm{H}$, $8-\mathrm{H}, 11-\mathrm{H}, 14-\mathrm{H}, 17-\mathrm{H}, 18-\mathrm{H}, \mathrm{OMe}), 3.884 .80(\mathrm{~m}, 38$ $\mathrm{H}, 1 \mathrm{c}-\mathrm{H}, 2 \mathrm{c}-\mathrm{H}, 3-\mathrm{H}, 4-\mathrm{H}, 6-\mathrm{H}, 7-\mathrm{H}, 9-\mathrm{H}, 10-\mathrm{H}, 12-\mathrm{H}$, $\left.13-\mathrm{H}, 15-\mathrm{H}, 16-\mathrm{H}, \mathrm{CH}_{2}-\mathrm{Ph}\right), 4.855 .11(\mathrm{~m}, 10 \mathrm{H}$, $\left.\mathrm{POCH}_{2}-\mathrm{Ph}\right), 6.686 .89\left(\mathrm{~m}, 8 \mathrm{H}, \mathrm{Ph}_{\mathrm{MPM}}\right), 7.017 .46$, $7.467 .70(\mathrm{~m}, 63 \mathrm{H}, \mathrm{Ph}) . \mathrm{C}_{137} \mathrm{H}_{160} \mathrm{NO}_{37} \mathrm{P}_{5} \mathrm{Si}(2595.7)$ : Calcd: C, 63.39; H, 6.21; N, 0.54. Found: C, 63.10; H, $6.46 ; \mathrm{N}, 0.55$.

\subsection{Pentaphosphate 41}

Compound 40 (433 mg, $0.17 \mathrm{mmol}$ ) was dissolved in THF $(15 \mathrm{~mL})$ and TBAF solution $(0.2 \mathrm{~mL}, 1.2$ equiv) was added. After $45 \mathrm{~min}$, the reaction mixture was diluted with EtOAc, washed with saturated $\mathrm{NaHCO}_{3}$ solution and water, and after drying over $\mathrm{MgSO}_{4}$ the solvent was removed in vacuo. Flash chromatography (toluene/acetone 1:1.5) yielded $41(335 \mathrm{mg}, 85 \%)$ as a colourless syrup. TLC (toluene/acetone 1:1.5): $R_{\mathrm{f}}=0.09, R_{\mathrm{f}}=0.20, R_{\mathrm{f}}=0.33$. $[\alpha]_{\mathrm{D}}+15.5(c=0.75$, ace- tone). ${ }^{1} \mathrm{H}$ NMR $\left(250 \mathrm{MHz}, \mathrm{CDCl}_{3}\right): \delta=1.862 .01(\mathrm{~m}, 3$ H, NHAc), 2.803 .20 (br s, $2 \mathrm{H}, \mathrm{OH}$ ), 3.413 .83 (m, 27 $\mathrm{H}, 3 \mathrm{c}-\mathrm{H}, 4 \mathrm{c}-\mathrm{H}, 5 \mathrm{c}-\mathrm{H}, 6 \mathrm{c}-\mathrm{H}, 1-\mathrm{H}, 2-\mathrm{H}, 5-\mathrm{H}, 8-\mathrm{H}, 11-\mathrm{H}$, 14-H, 17-H, 18-H, OMe), $3.894 .84(\mathrm{~m}, 38 \mathrm{H}, 1 \mathrm{c}-\mathrm{H}$, 2c-H, 3-H, 4-H, 6-H, 7-H, 9-H, 10-H, 12-H, 13-H, 15$\left.\mathrm{H}, 16-\mathrm{H}, \mathrm{CH}_{2}-\mathrm{Ph}\right), 4.905 .10\left(\mathrm{~m}, 10 \mathrm{H}, \mathrm{POCH}_{2}-\mathrm{Ph}\right)$, $6.716 .89\left(\mathrm{~m}, 8 \mathrm{H}, \mathrm{Ph}_{\mathrm{MPM}}\right), 7.087 .45(\mathrm{~m}, 53 \mathrm{H}, \mathrm{Ph})$.

\subsection{Heptaphosphate 42}

Compound 42 was synthesized following the general coupling procedure. Compound $\mathbf{4 1}$ (320 mg, $0.136 \mathrm{mmol})$, tetrazole $(19 \mathrm{mg}, 0.272 \mathrm{mmol})$ and phosphite amide $3(570 \mathrm{mg}, 2.5$ equiv) were dissolved in dry $\mathrm{CH}_{2} \mathrm{Cl}_{2}(10 \mathrm{~mL})$. Oxidation was achieved with $t$ $\mathrm{BuO}_{2} \mathrm{H}(1 \mathrm{~mL})$. Flash chromatography (toluene/acetone $3: 1 \rightarrow 1.5: 1)$ yielded $\mathbf{4 2}(520 \mathrm{mg}, 71 \%)$ as a colourless syrup. TLC (toluene/acetone 2:1): $R_{\mathrm{f}}=0.44, R_{\mathrm{f}}=0.51$, $R_{\mathrm{f}}=0.59 . \quad[\alpha]_{\mathrm{D}}+10.34$ (c 0.75 , acetone). ${ }^{1} \mathrm{H}$ NMR $\left(600 \mathrm{MHz}, \mathrm{CDCl}_{3}\right): \delta=0.800 .91(\mathrm{t}, 12 \mathrm{H}, \mathrm{Me}), 1.09$ $1.35\left(\mathrm{~m}, 80 \mathrm{H}, \mathrm{CH}_{2}\right.$-chain), $1.441 .52(\mathrm{~m}, 8 \mathrm{H}$, $\mathrm{COCH}_{2} \mathrm{CH}_{2} \mathrm{R}$ ), 1.871 .98 (m, $\left.3 \mathrm{H}, \mathrm{NHAc}\right), 2.092 .28$ $\left(\mathrm{m}, 8 \mathrm{H}, \mathrm{COCH}_{2} \mathrm{CH}_{2} \mathrm{R}\right), 3.31,3.39(\mathrm{~m}, 4 \mathrm{H}, 2 \mathrm{a}-\mathrm{H}, 2 \mathrm{~b}-$ $\mathrm{H}), 3.33(\mathrm{~m}, 2 \mathrm{H}, 5 \mathrm{a} / \mathrm{b}-\mathrm{H}), 3,36\left(\mathrm{~m}, 2 \mathrm{H}, 1^{\prime}-\mathrm{H}\right), 3.38$ (m, $2 \mathrm{H}, 5 \mathrm{a} / \mathrm{b}-\mathrm{H}), 3.45(\mathrm{~m}, 4 \mathrm{H}, 4 \mathrm{a}-\mathrm{H}, 4 \mathrm{~b}-\mathrm{H}), 3.56(\mathrm{~m}$, $1 \mathrm{H}, 6 \mathrm{c}-\mathrm{H}), 3.58(\mathrm{~m}, 4 \mathrm{H}, 3 \mathrm{a}-\mathrm{H}, 3 \mathrm{~b}-\mathrm{H}), 3.60(\mathrm{~m}, 2 \mathrm{H}$, $6 \mathrm{a}-\mathrm{H}), 3.62(\mathrm{~m}, 6 \mathrm{H}, 2-\mathrm{H}, 5-\mathrm{H}, 8-\mathrm{H}, 11-\mathrm{H}, 14-\mathrm{H}, 17-$ $\mathrm{H}), 3.64(\mathrm{~m}, 1 \mathrm{H}, 6 \mathrm{c}-\mathrm{H}), 3.66(\mathrm{~m}, 12 \mathrm{H}, \mathrm{OMe}), 3.68$ $(\mathrm{m}, 1 \mathrm{H}, 3 \mathrm{c}-\mathrm{H}), 3.69(\mathrm{~m}, 1 \mathrm{H}, 4 \mathrm{c}-\mathrm{H}), 3.77(\mathrm{~m}, 1 \mathrm{H}, 5 \mathrm{c}-$ $\mathrm{H}), 3.84\left(\mathrm{~m}, 2 \mathrm{H}, 1^{\prime}-\mathrm{H}\right), 3.95,4.03(\mathrm{~m}, 24 \mathrm{H}, 1-\mathrm{H}, 3-\mathrm{H}$, 4- $\mathrm{H}, 6-\mathrm{H}, 7-\mathrm{H}, 9-\mathrm{H}, 10-\mathrm{H}, 12-\mathrm{H}, 13-\mathrm{H}, 15-\mathrm{H}, 16-\mathrm{H}$, 18-H), $4.06\left(\mathrm{~m}, 2 \mathrm{H}, 3^{\prime}-\mathrm{H}\right), 4.10(\mathrm{~m}, 2 \mathrm{H}, 6 \mathrm{a}-\mathrm{H}), 4.15$ (m, $\left.2 \mathrm{H}, 3^{\prime}-\mathrm{H}\right), 4.18(\mathrm{~m}, 2 \mathrm{H}, 6 \mathrm{~b}-\mathrm{H}), 4.21(\mathrm{~m}, 2 \mathrm{H}, 1 \mathrm{a}-$ H), $4.23(\mathrm{~m}, 2 \mathrm{H}, 6 \mathrm{~b}-\mathrm{H}), 4.33(\mathrm{~m}, 1 \mathrm{H}, 2 \mathrm{c}-\mathrm{H}), 4.42(\mathrm{~m}$, $2 \mathrm{H}, 1 \mathrm{~b}-\mathrm{H}), 4.69 / 4.73(\mathrm{~m}, 1 \mathrm{H}, 1 \mathrm{c}-\mathrm{H}), 4.32-4.91(\mathrm{~m}, 40$ $\left.\mathrm{H}, \mathrm{CH}_{2} \mathrm{Ph}\right), 4.94\left(\mathrm{~m}, 14 \mathrm{H}, \mathrm{POCH} \mathrm{H}_{2} \mathrm{Ph}\right), 5.07(\mathrm{~m}, 2 \mathrm{H}$, $\left.2^{\prime}-\mathrm{H}\right), 6.686 .81\left(\mathrm{~m}, 8 \mathrm{H}, \mathrm{Ph}_{\mathrm{MPM}}\right), 7.057 .35$ (m, 123 $\mathrm{H}, \mathrm{Ph}) .{ }^{13} \mathrm{C}$ NMR $\left(150.9 \mathrm{MHz}, \mathrm{CDCl}_{3}\right): \delta=53.0(1 \mathrm{C}$, $\mathrm{C}-2 \mathrm{c}), 55.7$ (4C, OMe), 63.1 (2 C, C-3'), 66.0 (12 C, $\left.\mathrm{C} \mathrm{CH}_{2-\mathrm{Glyc}}\right), 66.8$ (2 C, C-6b), $68.4\left(2 \mathrm{C}, \mathrm{C}-1^{\prime}\right), 68.9$ (1 C, C-6c), 69.0 (2 C, C-6a), 69.7 (7 C, $\mathrm{POCH}_{2} \mathrm{Ph}$ ), 69.9 (2 C, C-2'), $72.078 .0\left(20 \mathrm{C}, \mathrm{CH}_{2} \mathrm{Ph}\right), 72.2(1 \mathrm{C}, \mathrm{C}-5 \mathrm{c})$, $74.0(2 \mathrm{C}, \mathrm{C}-5 \mathrm{a} / \mathrm{b}), 75.5$ (2 C, C-4a/b), 75.9 (6 C, C-2, C-5, C-8, C-11, C-14, C-17), 77.3 (2 C, C-4a/b), 78.0 (1 C, C-4c), 78.3 (2 C, C-5a/b), 81.4 (1 C, C-3c), 82.2 (4 C, C-2a, C-2b), 84.9 (4 C, C-3a, C-3b), 101.0 (1 C, C-1c), 104.0 (2 C, C-1a), 104.2 (2 C, C-1b). MALDIMS (positive Mode, Matrix $p$-nitroaniline+NaI, THF): $[\mathrm{M}+\mathrm{Na}]^{+}, \quad m / z=5440.1 ; \quad$ found: $\quad m / z=5445.6$. $\mathrm{C}_{305} \mathrm{H}_{384} \mathrm{NO}_{71} \mathrm{P}_{7}$ (5417.1): Calcd: C, 67.62; H, 7.14; N, 0.26. Found: C, 67.35; H, 7.32; N, 0.20.

\subsection{Heptaphosphate 43}

Compound 43 was synthesized following the same procedure used for 22. Compound $\mathbf{4 2}(476 \mathrm{mg}, 0.088 \mathrm{mmol})$ was dissolved in $\mathrm{CH}_{3} \mathrm{CN} /$ toluene/ $\mathrm{H}_{2} \mathrm{O}(60: 3: 4,15 \mathrm{~mL})$. Flash chromatography (toluene/acetone 1:1. $\rightarrow 1: 3$ ) yielded $43(330 \mathrm{mg}, 76 \%)$ as a colourless syrup. TLC (toluene/acetone 1:1.5): $R_{\mathrm{f}}=0.44, R_{\mathrm{f}}=0.53 .[\alpha]_{\mathrm{D}}+12.6$ (c 0.5 , acetone). ${ }^{1} \mathrm{H}$ NMR $\left(600 \mathrm{MHz}, \mathrm{CDCl}_{3}\right)$ : $\delta=0.800 .92(\mathrm{t}, 12 \mathrm{H}, \mathrm{Me}), 1.081 .36\left(\mathrm{~m}, 80 \mathrm{H}, \mathrm{CH}_{2-}\right.$ chain), $1.431 .59\left(\mathrm{~m}, 8 \mathrm{H}, \mathrm{COCH}_{2} \mathrm{CH}_{2} \mathrm{R}\right), 1.821 .97$ 
(m, $3 \mathrm{H}, \mathrm{NHAc}), 2.112 .27\left(\mathrm{~m}, 8 \mathrm{H}, \mathrm{COCH}_{2} \mathrm{CH}_{2} \mathrm{R}\right)$, 3.34 (m, $2 \mathrm{H}, 5 \mathrm{a} / \mathrm{b}-\mathrm{H}), 3.35$ (m, $4 \mathrm{H}, 2 \mathrm{a}-\mathrm{H}, 2 \mathrm{~b}-\mathrm{H}), 3.37$ $(\mathrm{m}, 2 \mathrm{H}, 5 \mathrm{a} / \mathrm{b}-\mathrm{H}), 3.38\left(\mathrm{~m}, 2 \mathrm{H}, 1^{\prime}-\mathrm{H}\right), 3.46(\mathrm{~m}, 2 \mathrm{H}$, 4a/b-H), 3.47 (m, $2 \mathrm{H}, 4 \mathrm{a} / \mathrm{b}-\mathrm{H}), 3.56(\mathrm{~m}, 1 \mathrm{H}, 6 \mathrm{c}-\mathrm{H})$, $3.58(\mathrm{~m}, 4 \mathrm{H}, 3 \mathrm{a}-\mathrm{H}, 3 \mathrm{~b}-\mathrm{H}), 3.59(\mathrm{~m}, 2 \mathrm{H}, 6 \mathrm{a}-\mathrm{H}), 3.64$ (m, $1 \mathrm{H}, 6 \mathrm{c}-\mathrm{H}), 3.67(\mathrm{~m}, 2 \mathrm{H}, 2-\mathrm{H}, 17-\mathrm{H}), 3.68(\mathrm{~m}, 1$ $\mathrm{H}, 3 \mathrm{c}-\mathrm{H}), 3.69(\mathrm{~m}, 1 \mathrm{H}, 4 \mathrm{c}-\mathrm{H}), 3.80(\mathrm{~m}, 1 \mathrm{H}, 5 \mathrm{c}-\mathrm{H})$, $3.85\left(\mathrm{~m}, 2 \mathrm{H}, 1^{\prime}-\mathrm{H}\right), 3.94(\mathrm{~m}, 4-\mathrm{H}, 6-\mathrm{H}, 7-\mathrm{H}, 9-\mathrm{H}, 10-$ $\mathrm{H}, 12-\mathrm{H}, 13-\mathrm{H}, 15-\mathrm{H}), 3.99(\mathrm{~m}, 4 \mathrm{H}, 1-\mathrm{H}, 3-\mathrm{H}, 16-\mathrm{H}$, $18-\mathrm{H}), 4.07\left(\mathrm{~m}, 2 \mathrm{H}, 3^{\prime}-\mathrm{H}\right), 4.08(\mathrm{~m}, 4 \mathrm{H}, 1-\mathrm{H}, 3-\mathrm{H}$, 16-H, 18-H), $4.10(\mathrm{~m}, 2 \mathrm{H}, 6 \mathrm{a}-\mathrm{H}), 4.15\left(\mathrm{~m}, 2 \mathrm{H}, 3^{\prime}-\mathrm{H}\right)$, $4.16(\mathrm{~m}, 2 \mathrm{H}, 6 \mathrm{~b}-\mathrm{H}), 4.22(\mathrm{~m}, 2 \mathrm{H}, 1 \mathrm{a}-\mathrm{H}), 4.24(\mathrm{~m}, 2$ $\mathrm{H}, 6 \mathrm{~b}-\mathrm{H}), 4.31(\mathrm{~m}, 1 \mathrm{H}, 2 \mathrm{c}-\mathrm{H}), 4.34-4.93(\mathrm{~m}, 32 \mathrm{H}$, $\left.\mathrm{CH}_{2} \mathrm{Ph}\right), 4.42(\mathrm{~m}, 2 \mathrm{H}, 1 \mathrm{~b}-\mathrm{H}), 4.83(\mathrm{~m}, 1 \mathrm{H}, 1 \mathrm{c}-\mathrm{H})$, $5.01\left(\mathrm{~m}, 14 \mathrm{H}, \mathrm{POCH} \mathrm{H}_{2} \mathrm{Ph}\right), 5.08\left(\mathrm{~m}, 2 \mathrm{H}, 2^{\prime}-\mathrm{H}\right), 7.05$ $7.47(\mathrm{~m}, 115 \mathrm{H}, \mathrm{Ph}) .{ }^{13} \mathrm{C}$ NMR $\left(150.9 \mathrm{MHz}, \mathrm{CDCl}_{3}\right)$ : $\delta=53.0(1 \mathrm{C}, \mathrm{C}-2 \mathrm{c}), 63.0\left(2 \mathrm{C}, \mathrm{C}-3^{\prime}\right), 66.0(4 \mathrm{C}, \mathrm{C}-1$, C-3, C-16, C-18), 66.8 (2 C, C-6b), 68.3 (2 C, C-1'), $68.4\left(\mathrm{CH}_{2-\mathrm{Glyc}}-\mathrm{OH}\right), 68.9$ (1 C, C-6c), 69.0 (2 C, C-6a), $70.0\left(7 \mathrm{C}, \mathrm{POCH}_{2} \mathrm{Ph}\right), 70.1\left(2 \mathrm{C}, \mathrm{C}-2^{\prime}\right), 72.0-76.4(16$ $\left.\mathrm{C}, \mathrm{CH}_{2} \mathrm{Ph}\right), 72.1$ (1 C, C-5c), 73.9 (2 C, C-5a/b), 75.5 (2 C, C-4a/b), 76.0 (2 C, C-2, C-17), 77.3 (2 C, C-4a/ b), 78.1 (1 C, C-4c), 78.3 (2 C, C-5a/b), 81.1 (1 C, C3c), 82.2 (4 C, C-2a, C-2b), 84.9 (4 C, C-3a, C-3b), 99.9 (1 C, C-1c), 104.0 (2 C, C-1a), 104.2 (2 C, C-1b). MALDI-MS (positive Mode, Matrix $p$-nitroaniline+NaI, THF): $[\mathrm{M}+\mathrm{Na}]^{+}, \mathrm{m} / z=4959.5$; found: $\mathrm{m} /$ $z=$ 4966.1. $\mathrm{C}_{273} \mathrm{H}_{352} \mathrm{NO}_{67} \mathrm{P}_{7}$ (4936.5): Calcd: $\mathrm{C}, 66.42$; H, 7.19; N, 0.28. Found: C, 66.30; H, 7.38; N, 0.24.

\subsection{Fully protected target molecule 44}

Compound 44 was synthesized following the procedure used for 23a. Compound 43 (296 mg, $0.060 \mathrm{mmol}$ ) gave 44 (170 mg, 49\%, mixture of diastereomers) as a colourless syrup. TLC (toluene/acetone 1:1): $\quad R_{\mathrm{f}}=0.60$, $R_{\mathrm{f}}=0.67 .[\alpha]_{\mathrm{D}}+12.45$ (c 0.48 , acetone). ${ }^{1} \mathrm{H}$ NMR $\left(600 \mathrm{MHz}, \mathrm{CDCl}_{3}\right): \delta=0.800 .94(\mathrm{t}, 12 \mathrm{H}, \mathrm{Me}), 1.07$ $1.38\left(\mathrm{~m}, 92 \mathrm{H}, \mathrm{CH}_{2}\right.$-chain, Ala-Me), 1.451 .59 (m, 8 $\left.\mathrm{H}, \mathrm{COCH}_{2} \mathrm{CH}_{2} \mathrm{R}\right), 1.851 .97(\mathrm{~m}, 3 \mathrm{H}, \mathrm{NHAc}), 2.11$ $2.28\left(\mathrm{~m}, 8 \mathrm{H}, \mathrm{COCH}_{2} \mathrm{CH}_{2} \mathrm{R}\right), 3.33(\mathrm{~m}, 2 \mathrm{H}, 5 \mathrm{a} / \mathrm{b}-\mathrm{H})$, $3.34(\mathrm{~m}, 4 \mathrm{H}, 2 \mathrm{a}-\mathrm{H}, 2 \mathrm{~b}-\mathrm{H}), 3.36\left(\mathrm{~m}, 4 \mathrm{H}, 5 \mathrm{a} / \mathrm{b}-\mathrm{H}, 1^{\prime}{ }_{-}\right.$ $\mathrm{H}), 3.46(\mathrm{~m}, 4 \mathrm{H}, 4 \mathrm{a}-\mathrm{H}, 4 \mathrm{~b}-\mathrm{H}), 3.58(\mathrm{~m}, 4 \mathrm{H}, 3 \mathrm{a}-\mathrm{H}$, 3b-H), $3.60(\mathrm{~m}, 4 \mathrm{H}, 6 \mathrm{c}-\mathrm{H}, 6 \mathrm{a}-\mathrm{H}), 3.64(\mathrm{~m}, 2 \mathrm{H}, 2-\mathrm{H}$, $17-\mathrm{H}), 3.68(\mathrm{~m}, 1 \mathrm{H}, 3 \mathrm{c}-\mathrm{H}), 3.70(\mathrm{~m}, 1 \mathrm{H}, 4 \mathrm{c}-\mathrm{H}), 3.76$ $(\mathrm{m}, 1 \mathrm{H}, 5 \mathrm{c}-\mathrm{H}), 3.84\left(\mathrm{~m}, 2 \mathrm{H}, 1^{\prime}-\mathrm{H}\right), 4,00(\mathrm{~m}, 24 \mathrm{H}, 1-$ $\mathrm{H}, 3-\mathrm{H}, 4-\mathrm{H}, 6-\mathrm{H}, 7-\mathrm{H}, 9-\mathrm{H}, 10-\mathrm{H}, 12-\mathrm{H}, 13-\mathrm{H}, 15-\mathrm{H}$, 16-H, 18-H), $4.09\left(\mathrm{~m}, 2 \mathrm{H}, 3^{\prime}-\mathrm{H}\right), 4.11(\mathrm{~m}, 2 \mathrm{H}, 6 \mathrm{a}-\mathrm{H})$, $4.15\left(\mathrm{~m}, 2 \mathrm{H}, 3^{\prime}-\mathrm{H}\right), 4.18(\mathrm{~m}, 2 \mathrm{H}, 6 \mathrm{~b}-\mathrm{H}), 4.22(\mathrm{~m}, 2$ $\mathrm{H}, 1 \mathrm{a}-\mathrm{H}), 4.24(\mathrm{~m}, 2 \mathrm{H}, 6 \mathrm{~b}-\mathrm{H}), 4.33(\mathrm{~m}, 4 \mathrm{H}, \mathrm{CH}-$ NHCbz), $4.35(\mathrm{~m}, 1 \mathrm{H}, 2 \mathrm{c}-\mathrm{H}), 4.34-4.93(\mathrm{~m}, 32 \mathrm{H}$, $\left.\mathrm{CH}_{2} \mathrm{Ph}\right), 4.42(\mathrm{~m}, 2 \mathrm{H}, 1 \mathrm{~b}-\mathrm{H}), 4.78(\mathrm{~m}, 1 \mathrm{H}, 1 \mathrm{c}-\mathrm{H})$, $4.96\left(\mathrm{~m}, 4 \mathrm{H}, \mathrm{CH}_{2}-\mathrm{Cbz}\right), 4.98\left(\mathrm{~m}, 14 \mathrm{H}, \mathrm{POCH}_{2} \mathrm{Ph}\right)$, $5.03\left(\mathrm{~m}, 4 \mathrm{H}, \mathrm{CH}_{2}-\mathrm{Cbz}\right), 5.08\left(\mathrm{~m}, 2 \mathrm{H}, 2^{\prime}-\mathrm{H}\right), 5.09(\mathrm{~m}$, $4 \mathrm{H}, 5,8,11,14-\mathrm{H}), 5.76-6.18$ (bs, NH), 7.037 .46 (m, $135 \mathrm{H}, \mathrm{Ph}) .{ }^{13} \mathrm{C}$ NMR $\left(150.9 \mathrm{MHz}, \mathrm{CDCl}_{3}\right): \delta=49.9$ (4 C, CH-NHCbz), 53.0 (1 C, C-2c), $63.0\left(2 \mathrm{C}, \mathrm{C}-3^{\prime}\right)$, 65.3 (12 C, C-1, C-3, C-4, C-6, C-7, C-9, C-10, C-12, C-13, C-15, C-16, C-18), 66.8 (2 C, C-6b), 67.1 (4 C, $\left.\mathrm{CH}_{2}-\mathrm{Cbz}\right), 68.3$ (2 C, C-1'), 68.8 (1 C, C-6c), 68.9 (2 $\mathrm{C}, \mathrm{C}-6 \mathrm{a}), 70.0\left(2 \mathrm{C}, \mathrm{C}-2^{\prime}\right), 70.3\left(7 \mathrm{C}, \mathrm{POCH}_{2} \mathrm{Ph}\right), 71.0$ (4 C, C-5, C-8, C-11, C-14), 72.0 (1 C, C-5c), 73.9 (2 C, C-5a/b), $72.575 .9\left(16 \mathrm{C}, \mathrm{CH}_{2} \mathrm{Ph}\right), 73.8(2 \mathrm{C}, \mathrm{C}-5 \mathrm{a} /$ b), 75.3 (2 C, C-4a/b), 76.1 (2 C, C-2, C-17), 77.1 (2 C, C-4a/b), 77.9 (1 C, C-4c), 78.3 (2 C, C-5a/b), 81.2 (1 C, C-3c), 82.1 (4 C, C-2a, C-2b), 84.7 (4 C, C-3a, C3b), 100.6 (1 C, C-1c), 103.8 (2 C, C-1a), 104.2 (2 C, $\mathrm{C}-1 \mathrm{~b})$. MALDI-MS (positive Mode, Matrix $p$-nitroaniline+NaI, THF): $[(\mathrm{M}-\mathrm{H})+2 \mathrm{Na}]^{+}, m / z=5802.3$; found: $m / z=5801.3 . \quad \mathrm{C}_{317} \mathrm{H}_{396} \mathrm{~N}_{5} \mathrm{O}_{79} \mathrm{P}_{7}$ (5757.34): Calcd: $\mathrm{C}$, 66.13; H, 6.93; N, 1.22. Found: C, 65.94; H, 7.08; N, 1.20 .

\subsection{Target molecule 2}

Compound 2 was synthesized following the procedure used for 1a. Compound $44(107 \mathrm{mg}, 0.019 \mathrm{mmol})$ yielded $2(19 \mathrm{mg}, 16 \%)$ as white powder. ${ }^{1} \mathrm{H}$ NMR $\left(600 \mathrm{MHz}, \mathrm{D}_{2} \mathrm{O}\right): \delta=0.780 .96(\mathrm{~m}, 12 \mathrm{H}, \mathrm{Me}), 1.13$ $1.46\left(\mathrm{~m}, 80 \mathrm{H}, \mathrm{CH}_{2}\right.$-chain), $1.551 .74(\mathrm{~m}, 20 \mathrm{H}$, Ala$\mathrm{Me}, \mathrm{COCH}_{2} \mathrm{CH}_{2} \mathrm{R}$ ), 2.11 (s, $3 \mathrm{H}, \mathrm{NHAc}$ ), 2.272 .49 $\left(\mathrm{m}, 8 \mathrm{H}, \mathrm{COCH}_{2} \mathrm{CH}_{2} \mathrm{R}\right), 3.404 .60(\mathrm{~m}, 72 \mathrm{H}), 5.10(\mathrm{~m}$, $1 \mathrm{H}, 1 \mathrm{c}-\mathrm{H}), 5.315 .45\left(\mathrm{~m}, 6 \mathrm{H}, \mathrm{CH}-\mathrm{Ala}, 22^{\prime}-\mathrm{H}\right) .{ }^{13} \mathrm{C}$ NMR (150.9 MHz, $\mathrm{D}_{2} \mathrm{O}$ ): $\delta=14.2(4 \mathrm{C}, \mathrm{Me}), 15.9$ (4 $\mathrm{C}$, Ala-Me), 22.6 (1 C, NHAc), 25.2 (4 C, $\mathrm{COCH}_{2} \mathrm{CH}_{2} \mathrm{R}$ ), 30.9 (40 C, $\mathrm{CH}_{2}$-chain), 34.7 (4 C, $\mathrm{COCH}_{2} \mathrm{R}$ ), 49.1 (4 C, $\mathrm{CHNH}_{3}{ }^{+}, 60.9$ (1 C, C-6c), 64.0 ( $\mathrm{C} \mathrm{CH}_{2 \text {-Glyc }}$, 66.4 ( $\mathrm{C} \mathrm{CH}_{2 \text {-Glyc }}$, 74.1 (4C, $\mathrm{CH}$-Ala). MALDI-MS (negative Mode, Matrix THAP, $\mathrm{CH}_{3} \mathrm{CN}$ / $\mathrm{H}_{2} \mathrm{O}$ 3:2): $[\mathrm{M}-\mathrm{H}], \mathrm{m} / \mathrm{z}=3147.0$; found: $\mathrm{m} / \mathrm{z}=3146.0$; [(M-Ala)-H],$m / z=3074.9$; found: $m / z=3075.2$.

\subsection{Measurement of biological activity}

Heparinized whole blood from healthy donors controlled by differential blood cell count was diluted 5-fold with RPMI 1640 (BioWhittaker Europe) and stimulated overnight $\left(37^{\circ} \mathrm{C}, 5 \% \mathrm{CO}_{2}\right)$ in polypropylene cups with equimolar concentrations of $\mathbf{1 a}$ and $\mathbf{2}$. Cytokines were measured in the supernatants using ELISA antibody pairs against TNF $\alpha$ and IL-8 (Endogen, Pierce, Perbio Science, Bonn, Germany).

\section{Acknowledgments}

This work was supported by the Deutsche Forschungsgemeinschaft and the Fonds der Chemischen Industrie. We thank L. Cobianchi for help in separating the final products by HI-chromatography, and Prof. A. Geyer and A. Friemel for the help in the structural assignments of the products by NMR experiments.

\section{References and notes}

1. Rietschel, E. T.; Brade, H.; Holst, O.; Brade, L.; Müller Loennies, S.; Mamat, U.; Zähringer, U.; Bekcmann, F.; Seydel, U.; Brandenburg, K.; Ulmer, A.; Mattern, T.; Heine, H.; Schletter, J.; Hauschildt, S.; Loppnow, H.; Schönbeck, U.; Flad, H. D.; Schade, U. F.; DiPadova, F.; Kusumoto, S.; Schumann, R. R. Curr. Top. Microbiol. Immunol. 1996, 216, 3981.

2. Seydel, U.; Oikawa, M.; Fukase, K.; Kusumoto, S.; Brandenburg, K. Eur. J. Biochem. 2000, 267, 30323039.

3. Morath, S.; Stadelmaier, A.; Geyer, A.; Schmidt, R. R.; Hartung, T. J. Exp. Med. 2002, 195, 16351640. 
4. (a) Stadelmaier, A.; Morath, S.; Hartung, T.; Schmidt, R. R. Angew. Chem. 2003, 115, 945 949; (b). Angew. Chem. Int. Ed. 2003, 42, 916920.

5. Deininger, S.; Stadelmaier, A.; von Aulock, S.; Morath, S.; Schmidt, R. R.; Hartung, T. J. Immunol 2003, 170, 41344138.

6. De Vos, W. M. Proc. Natl. Acad. Sci. U.S.A. 2005, 102, 10763 10764, and references therein.

7. File, T. M.; Tan, J. S. Am. J. Surg. 1995, 169, 2733.

8. Lowy, F. D. N. Engl. J. Med. 1998, 339, 520532.

9. Murray, B. E. N. Engl. J. Med. 1994, 330, 12291230.

10. Fischer, W. Med. Microbiol. Immunol. 1994, 183, 6176.

11. Morath, S.; Geyer, A.; Hartung, T. J. Exp. Med. 2001, 193, 393397.

12. Stadelmaier, A. Dissertation, Universität Konstanz, 2003.

13. Figueroa Perez, I.; Stadelmaier, A.; Morath, S.; Hartung, T.; Schmidt, R. R. Tetrahedron: Asym. 2005, 16, 493506.
14. Increased activity of bound LTA was attributed to receptor clustering already previously Mancuso, G.; Tomasello, F.; Ofek, I.; Teti, G. Infect. Immun. 1994, 62, 14701473.

15. For a short communication of this work, see Ref. 4.

16. Hirth, G.; Walther, W. Helv. Chim. Acta 1985, 68, 1863 1871.

17. Bannwarth, W.; Trzeciak, A. Helv. Chim. Acta 1987, 70, 175186.

18. Coste, J.; Le Nguyen, D.; Castro, B. Tetrahedron Lett. 1990, 31, 205208.

19. Fieser, L. F.; Fieser, M.. In Reagents for Organic Synthesis; Wiley: New York, 1967; Vol. 1, p. 782.

20. Corey, E. J.; Suggs, J. W. J. Org. Chem. 1973, 38, 3224.

21. Guivisdalsky, P. N.; Bittman, R. J. Am. Chem. Soc 1989, 111, 30773079 . 